\title{
An Experimental and Theoretical Investigation of Evaporating Meniscus Dynamics and Instabilities
}

by

\author{
John L. Polansky \\ A Dissertation submitted to \\ the Faculty of Graduate Studies and Research \\ in partial fulfilment of \\ the requirements for the degree of \\ Doctor of Philosophy \\ Department of Mechanical and Aerospace Engineering \\ Carleton University \\ Ottawa, Ontario, Canada
}

April 2016

Copyright (C)

2016 - John L. Polansky 
The undersigned recommend to the Faculty of Graduate Studies and Research acceptance of the Dissertation

\title{
An Experimental and Theoretical Investigation of Evaporating Meniscus Dynamics and Instabilities
}

\author{
Submitted by John L. Polansky \\ in partial fulfilment of the requirements for the degree of \\ Doctor of Philosophy
}

T. Kaya, Supervisor

M. Yaras, Department Chair

Carleton University

2016 


\section{Abstract}

High power density systems utilising phase change heat transfer devices such as heat pipes can be susceptible to evaporation driven meniscus dynamics and instability. To better understand this, an study of evaporating meniscus dynamics and instability was needed. A mathematical model describing evaporating meniscus dynamics was developed in which meniscus height was correlated with superheat. Subsequent validation experiments confirmed the model was consistent with the general trends including the superheat to meniscus height relation.

The study of evaporating meniscus instability was investigated using a one-sided model and a linear stability analysis. The analysis considered the effects of long range molecular forces, surface tension, vapour recoil, evaporation, thermocapillarity and viscous forces. The potential for instability was studied for three film geometries, for which the potential for instability was found to be spatially dependent for the curved cases, with perturbation growth rates increasing with superheat.

An experimental study of channel based evaporating meniscus instability was performed for eight channel widths and three fluids: n-pentane, iso-octane and acetone. The meniscus height to superheat correlation was used to infer the superheat at which the meniscus destabilised. The experiments revealed two kinds of instability. The first was localised to a narrow range of superheats and unique to the alkanes, the second common to all three fluids and sustained for higher superheats. The second kind of instability was found to require larger superheats for decreasing channel widths. 
"An expert is a person who has made all the mistakes that can be made in a very narrow field."

- Niels Bohr 


\section{Acknowledgments}

First and foremost, I would like to thank my thesis supervisor Professor Tarik Kaya for his unwaivering support and guidance throughout the course of my graduate studies. It is with his judicious application of wisdom that I have been able to grow as both a researcher and individual, for which I am truly grateful. Our many discussions and debates have been invaluable and it is my hope that our efforts will prove fruitful for many years to come.

I would like to extend many thanks to professors Bruce Burlton and Edgar Matida for their support, guidance and varied perspectives that helped me to see the problems in a new light. Furthermore, the time and assistance from the technical staff is greatly appreciated as their efforts helped me to bring my experimental ambitions into reality.

I would like to thank my father Steve and brother William for their continued support through my academic pursuits. And last, though certainly not least, I would like to thank my friends and colleagues: Sriram Venkataramanan, Ruslan Nasir, Alex Iska, Tommy Yuen, David Ouellette and many others too numerous to list for their valued insights, help and jokes along the way. 


\section{Table of Contents}

Abstract $\quad$ iii

Acknowledgments $\quad$ v

Table of Contents vi vi

List of Tables $\quad$ x

List of Figures $\quad$ xi

Nomenclature $\quad$ xiv

1 Introduction 1

1.1 Research motivation ................... 2

2 Literature survey $\quad 6$

2.1 The interface ........................ 6

2.1.1 The meniscus ................... 7

2.2 Thin-film model development . . . . . . . . . . . . . . . 12

2.2.1 Augmented Young-Laplace model . . . . . . . . . . . . . . . . 14

2.3 Evaporation .......................... 15

2.3.1 The Kelvin-Clapeyron model . . . . . . . . . . . . . 15

2.4 Numerical studies . . . . . . . . . . . . . . . . . . . . . . . 17 
2.4 .1 Continuum models . . . . . . . . . . . . . . . . . . . . 17

2.4.2 Behavioural characteristics . . . . . . . . . . . . . 18

2.5 Alternate models . . . . . . . . . . . . . . . . . . . . . . 19

2.5.1 Computational fluid dynamics . . . . . . . . . . . . . 20

2.5 .2 Molecular dynamics . . . . . . . . . . . . . . . . . . . . 22

2.6 Experimental investigations . . . . . . . . . . . . . . . 23

2.6 .1 Thin-film geometry . . . . . . . . . . . . . . . . . 23

2.6.2 Temperature profile . . . . . . . . . . . . . . . . . . . 24

2.6 .3 Velocity profile . . . . . . . . . . . . . 25

2.7 Meniscus stability . . . . . . . . . . . . . . . . . . . . . 26

2.7 .1 Meniscus dynamics . . . . . . . . . . . . . . . . . 27

2.7 .2 Meniscus stability . . . . . . . . . . . . . . . . . 28

2.8 Summary . . . . . . . . . . . . . . . . . . . . 35

3 Meniscus dynamics - model $\quad 37$

3.1 The flow domain $\ldots \ldots \ldots \ldots \ldots \ldots \ldots$

3.2 Generalised capillary flow . . . . . . . . . . . . . . . . . 39

3.2 .1 Averaged capillary flow . . . . . . . . . . . . . . . . 41

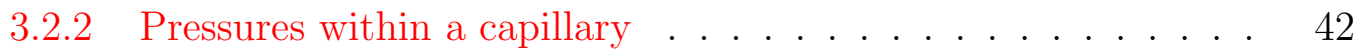

3.3 The interface $\ldots \ldots \ldots \ldots \ldots \ldots \ldots \ldots \ldots$

3.3 .1 Phase change model . . . . . . . . . . . . . . . . . 47

3.4 Steady state meniscus . . . . . . . . . . . . . . . 47

3.5 Numerical solution . . . . . . . . . . . . . . . . . . . . 48

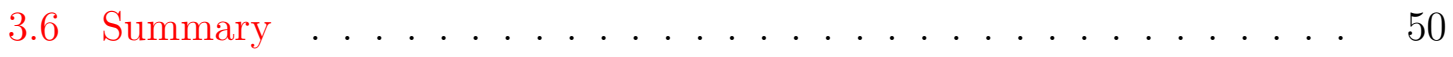

4 Meniscus dynamics - experiments $\quad 52$

4.1 Experimental setup . . . . . . . . . . . . . . . 52

4.2 Experimental procedure $\ldots \ldots \ldots \ldots \ldots \ldots \ldots$ 
4.3 Post processing techniques . . . . . . . . . . . . . . 57

4.4 Experimental results . . . . . . . . . . . . . . . . . . 58

4.4.1 Differences attributed to heating . . . . . . . . . . . 58

4.4.2 Differences attributed to the fluid . . . . . . . . . . . . . . 61

4.4.3 Factor of merit . . . . . . . . . . . . . . 63

4.5 Model validation . . . . . . . . . . . . . . . . . . . 64

4.5.1 Superheat determination . . . . . . . . . . . 66

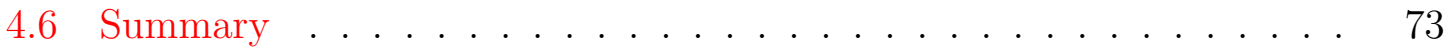

5 Meniscus instability - model $\quad 74$

5.1 Governing equations ................... 76

5.2 Scaling the equations . . . . . . . . . . . . . . 81

5.2.1 Reduction of the characteristic ratio . . . . . . . . . . . 84

5.3 Evolution equation ..................... 85

5.3.1 Linear stability model . . . . . . . . . . . . . . . . . 87

5.4 Film stability analysis . . . . . . . . . . . . . . . . . 89

5.4.1 Flat film ........................ 89

5.4 .2 Modified Young-Laplace . . . . . . . . . . . . . . . . . 91

5.4 .3 Curved thin-film base state . . . . . . . . . . . . 96

5.4 Thin-film stability . . . . . . . . . . . . . . 100

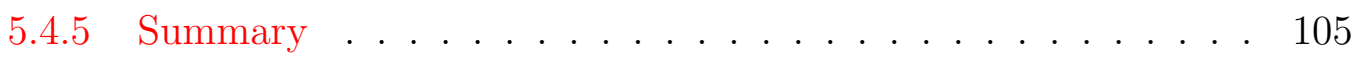

6 Meniscus instability - experiments 107

6.1 Experimental setup . . . . . . . . . . . . . . . . 107

6.2 Experimental procedure . . . . . . . . . . . . . . . 112

6.3 Superheat measurement options . . . . . . . . . . . . . . . . . . . 114

6.4 Superheat model . . . . . . . . . . . . . . . . . 116

6.5 Experimental results . . . . . . . . . . . . . . . . 117 
6.5.1 Instability nature . . . . . . . . . . . . . . . . 118

6.5.2 Instability onset . . . . . . . . . . . . . . . 121

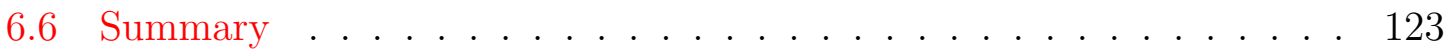

7 Concluding remarks $\quad 125$

7.1 Conclusions . . . . . . . . . . . . . . . . . . 125

7.2 Future work . . . . . . . . . . . . . . . . . 129

List of References $\quad 130$

$\begin{array}{ll}\text { Appendix A Velocity in Y } & 141\end{array}$

$\begin{array}{lll}\text { Appendix B Evolution equation } & 143\end{array}$

$\begin{array}{lll}\text { Appendix C Simplified evolution equation } & 150\end{array}$

$\begin{array}{ll}\text { Appendix D Perturbation growth rate } & 151\end{array}$ 


\section{List of Tables}

1 Fluid properties consistent with Ramon and Oron . . . . . . . . . 49

2 Capillary tube and lens pairings. . . . . . . . . . . . 54

3 Experimental parameters. ................ 56

4 Factor of merit for each fluid tested. . . . . . . . . . . . . . . 64 


\section{List of Figures}

1 A nucleate bubble forming on the bottom of a large reservoir. . . . . 2

2 A set of heat pipes on the Sample Analysis at Mars (SAM) payload for the Curiosity mission. . . . . . . . . . . . . . . . . . 3

3 The stages of liquid film rupture. . . . . . . . . . . . . . 4

4 Channel based meniscus and thin film region. . . . . . . . . . . . 8

$5 \quad$ Schematic illustrating the contact angle for an early thin-film analysis. 13

6 Perturbation and accommodation coefficient relation. . . . . . . . . 19

7 CFD analysis of capillary tubes in a gravitational field. . . . . . . . . 21

8 Thermochromic liquid crystal application in thin-film substrate temperature measurement. . . . . . . . . . . . . . . . 25

9 Coupled ring and point rupture modes. . . . . . . . . . . . . . . . 33

10 A cylindrical capillary tube with the associated liquid reservoir and meniscus. . . . . . . . . . . . . . . . . . . . . . . . . 38

11 A graphical representation of the potential wall slip. . . . . . . . . 40

12 Model verification for water. . . . . . . . . . . . . . 50

13 Experimental setup. . . . . . . . . . . . . . . 53

14 Internal experimental setup. . . . . . . . . . . . . . . . 55

$150.5 \mathrm{~mm}$ capillary tube with heated iso-octane. . . . . . . . . 59

$161.0 \mathrm{~mm}$ capillary tube with iso-octane. . . . . . . . . . . . 60

$172.0 \mathrm{~mm}$ capillary tube with iso-octane. . . . . . . . . . . 61 
18 Unheated $2.0 \mathrm{~mm}$ capillary tube meniscus heights. . . . . . . . . . . . 62

19 Heated (6 W) 2.0 mm capillary tube meniscus heights. . . . . . . . . 62

20 Unheated (blue) and heated at $6 \mathrm{~W}$ (red) capillary rise profiles for n-pentane in a $0.5 \mathrm{~mm}$ capillary tube. . . . . . . . . . . . . 67

21 Heated $1.0 \mathrm{~mm}$ capillary rise profiles for n-pentane at $6.0 \mathrm{~W}$. . . . . 67

22 Heated $2.0 \mathrm{~mm}$ capillary rise profiles for n-pentane at $6.0 \mathrm{~W} \ldots$. . . . 68

23 Heated $0.5 \mathrm{~mm}$ capillary rise profiles for iso-octane at $2.7 \mathrm{~W} \ldots$. . . . 69

24 Heated 1.0 mm capillary rise profiles for iso-octane at $2.7 \mathrm{~W}$. . . . . 69

25 Heated $2.0 \mathrm{~mm}$ capillary rise profiles for iso-octane at $2.7 \mathrm{~W}$. . . . . 70

26 Heated $0.5 \mathrm{~mm}$ capillary rise profiles for acetone at $0.7 \mathrm{~W}$. . . . . . 71

27 Heated $1.0 \mathrm{~mm}$ capillary rise profiles for acetone at $0.7 \mathrm{~W}$. . . . . . 71

28 Heated $2.0 \mathrm{~mm}$ capillary rise profiles for acetone at $0.7 \mathrm{~W}$. . . . . . 72

29 Channel based meniscus and thin-film region. . . . . . . . . . . . 75

30 Perturbation growth rates for an evaporating flat film. . . . . . . . . 91

31 Perturbation growth rates for an evaporating MYL film. . . . . . . . 93

32 3-D Perturbation growth rates for the first 10\% of an evaporating MYL

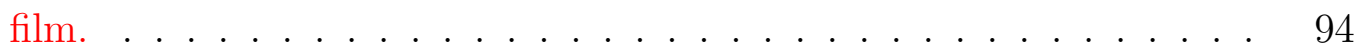

33 Side view of the $3-\mathrm{D}$ perturbation illustrating the growth rates sporadic

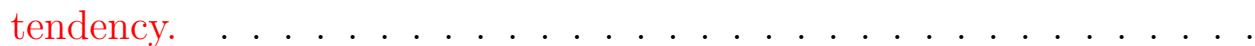

34 Side view of the $3-\mathrm{D}$ perturbation illustrating the trajectories of the seemingly sporadic points. . . . . . . . . . . . . . . . . 95

35 Thin-film thickness for a range of superheats. . . . . . . . . . . 97

36 Thin-film curvature for a range of superheats. . . . . . . . . . . . . 98

37 Thin-film third derivative for a range of superheats. . . . . . . . . . . 99

38 Thin-film fourth derivative for a range of superheats. . . . . . . . . . 99

39 Cutoff wavenumbers for a $100 \mu \mathrm{m}$ channel and n-pentane for a range of superheats. . . . . . . . . . . . . . . . . 100 
40 Cutoff wavenumbers based on $\Delta T$ and $x \ldots \ldots \ldots \ldots$. . . . . 101

41 Cutoff wavenumbers based on $k$ and $x \ldots \ldots \ldots \ldots \ldots$

42 Cutoff wavenumbers based on $k$ and $\Delta T \ldots \ldots \ldots \ldots \ldots$

43 Perturbation growth rates for $\mathrm{n}$-pentane and $k=0.15 \ldots \ldots \ldots$

44 Comparison of the peak thin-film properties and perturbation growth rate locations. . . . . . . . . . . . . . . . . . . 105

45 Experimental setup on the granite table. . . . . . . . . . . 108

46 Thermopile installation. . . . . . . . . . . . . . . . . . . . . . . . 109

47 Three experiment carousel. . . . . . . . . . . . . . . . . . . 110

48 Typical temperature channel distribution as seen from the thermopile array. . . . . . . . . . . . . . . . . . 111

49 Typical instability motion profiles for low superheat instability. . . . . 118

$50 \quad$ FFT data for the typical instability motion profiles. . . . . . . . . 119

51 Typical instability motion profiles. . . . . . . . . . . . . 120

52 Meniscus height at the onset of the second kind of instability for npentane, iso-octane and acetone. . . . . . . . . . . . . . . 122

53 Unstable superheats for the second kind of instability for n-pentane, iso-octane and acetone. . . . . . . . . . . . . . . . . . . . . 123 


\section{Nomenclature}

\section{Latin Characters}

\begin{tabular}{|c|c|c|}
\hline Symbol & Description & Units \\
\hline$a$ & Clapeyron evaporative mass flux coefficient & {$\left[\frac{k g}{m^{2} s K}\right]$} \\
\hline$A$ & Dispersion constant & {$[J]$} \\
\hline$A$ & Area & {$\left[m^{2}\right]$} \\
\hline $\mathcal{A}$ & Perturbation growth rate coefficient & {$[-]$} \\
\hline $\mathcal{B}$ & Perturbation growth rate coefficient & {$[-]$} \\
\hline$b$ & Kelvin evaporative mass flux coefficient & {$\left[\frac{s}{m}\right]$} \\
\hline$c_{p}$ & Specific heat & {$\left[\frac{J}{k g K}\right]$} \\
\hline$C$ & Accommodation coefficient & {$[-]$} \\
\hline $\mathcal{C}$ & Perturbation growth rate coefficient & {$[-]$} \\
\hline $\mathrm{Ca}$ & Capillary number & {$[-]$} \\
\hline$D$ & Difference or material derivative & {$[-]$} \\
\hline$D$ & Capillary tube diameter & {$[m]$} \\
\hline
\end{tabular}




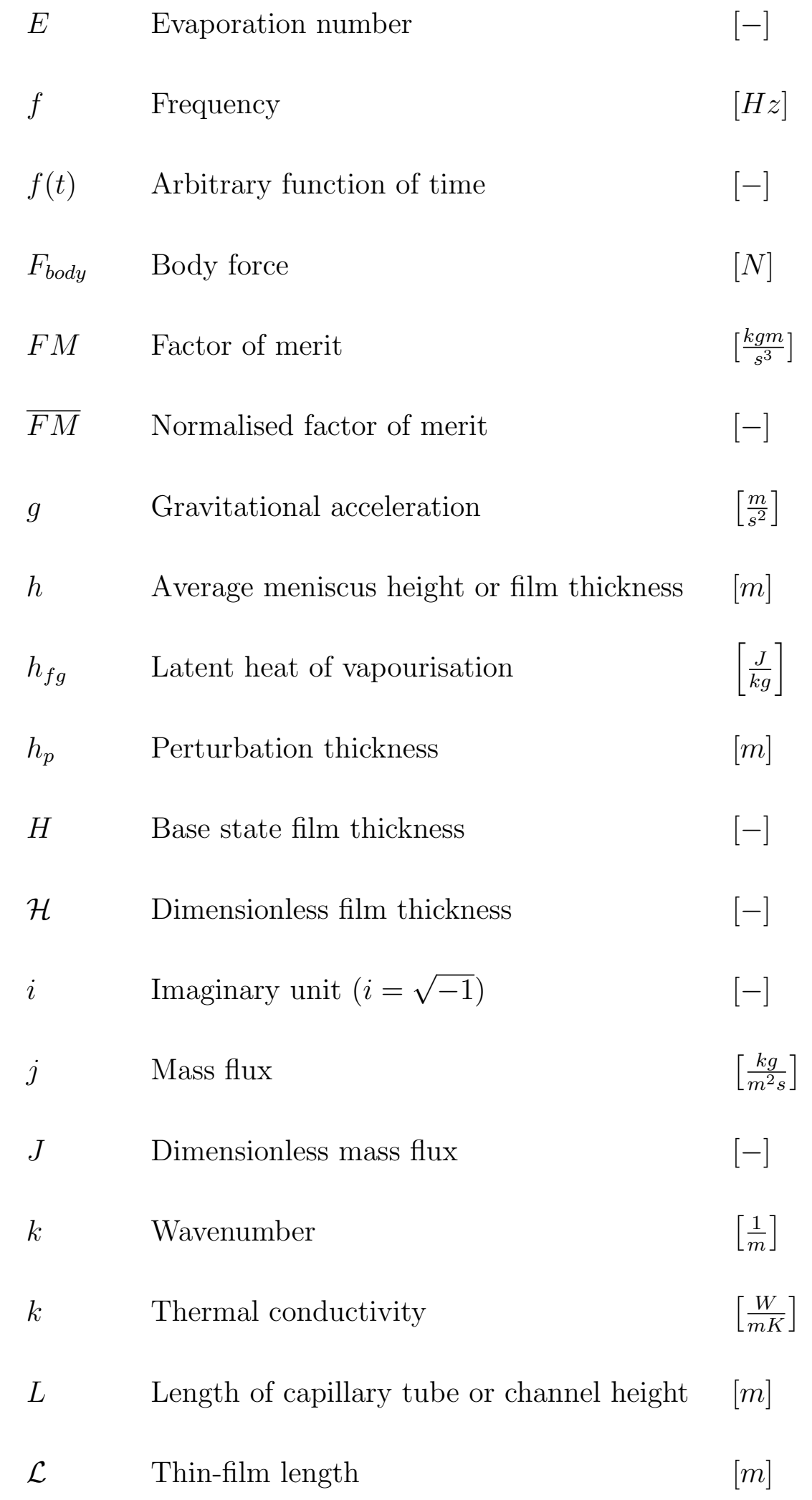




\begin{tabular}{|c|c|c|}
\hline$m$ & Mass & {$[\mathrm{kg}]$} \\
\hline$M$ & Molar mass & {$\left[\frac{\mathrm{kg}}{\mathrm{kmol}}\right]$} \\
\hline$M a$ & Marangoni number & {$[-]$} \\
\hline$\hat{\mathbf{n}}$ & Normal unit vector & {$[-]$} \\
\hline$O$ & Order of magnitude & {$[-]$} \\
\hline$p$ & Pressure & {$[P a]$} \\
\hline $\mathrm{p}$ & Pressure vector & {$[\mathrm{Pa}]$} \\
\hline$P$ & Dimensionless pressure & {$[-]$} \\
\hline$P e$ & Peclet number & {$[-]$} \\
\hline$r$ & Radial coordinate & {$[m]$} \\
\hline$R$ & Universal gas constant & {$\left[\frac{J}{m o l K}\right.$} \\
\hline$R$ & Capillary tube radius & {$[m]$} \\
\hline Re & Reynolds number & {$[-]$} \\
\hline$R e_{m}$ & Modified Reynolds number & {$[-]$} \\
\hline$t$ & Time & {$[s]$} \\
\hline$\hat{\mathrm{t}}$ & Tangential unit vector & {$[-]$} \\
\hline$T$ & Temperature & {$[K]$} \\
\hline $\mathbf{T}$ & Cauchy stress tensor & {$[P a]$} \\
\hline$u$ & Velocity in $\mathrm{x}$ or average velocity & {$\left[\frac{m}{s}\right]$} \\
\hline
\end{tabular}




\begin{tabular}{|c|c|c|}
\hline$U$ & Dimensionless velocity in $\mathrm{X}$ & {$[-]$} \\
\hline$v$ & Velocity in $y$ & {$\left[\frac{m}{s}\right]$} \\
\hline$V$ & Dimensionless velocity in $\mathrm{Y}$ & {$[-]$} \\
\hline $\mathbf{V}$ & Velocity vector & {$\left[\frac{m}{s}\right]$} \\
\hline$V_{l}$ & Molar volume & {$\left[\frac{\mathrm{m}^{3}}{\mathrm{~mol}}\right]$} \\
\hline$\forall$ & Volume & {$\left[m^{3}\right]$} \\
\hline$w$ & Velocity in $z$ & {$\left[\frac{m}{s}\right]$} \\
\hline $\bar{w}$ & Average meniscus velocity & {$\left[\frac{m}{s}\right]$} \\
\hline$W$ & Channel width & {$[m]$} \\
\hline$x$ & Coordinate in $\mathrm{x}$ & {$[m]$} \\
\hline$X$ & Dimensionless x coordinate & {$[-]$} \\
\hline$y$ & Coordinate in $y$ & {$[m]$} \\
\hline$Y$ & Dimensionless y coordinate & {$[-]$} \\
\hline
\end{tabular}

\section{Greek Characters}

\begin{tabular}{llc} 
Symbol & Description & Units \\
\hline$\alpha$ & Tangent to Cartesian x angle & {$[\mathrm{rad}]$} \\
$\gamma$ & Surface tension thermal coefficient & {$\left[\frac{N}{m K}\right]$} \\
$\Gamma$ & Dispersion number & {$[-]$}
\end{tabular}




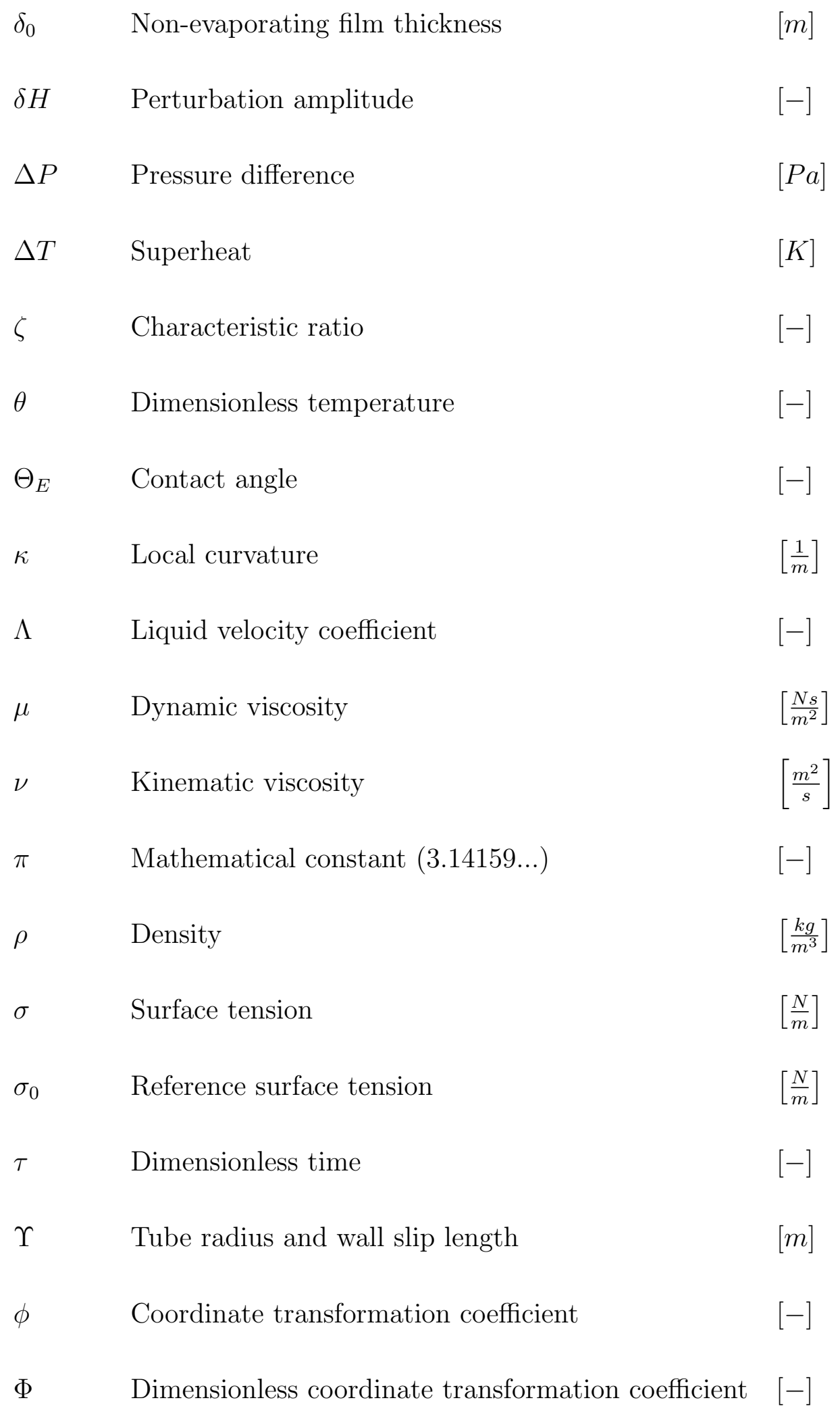


$\psi \quad$ Change of variable

\section{Subscripts}

\begin{tabular}{|c|c|c|}
\hline Symbol & Description & Units \\
\hline$a m b$ & Ambient & {$[-]$} \\
\hline$c$ & Characteristic or cutoff & {$[-]$} \\
\hline cap & Capillary & {$[-]$} \\
\hline$d$ & Disjoining & {$[-]$} \\
\hline $\exp$ & Experimental data & {$[-]$} \\
\hline$h$ & Hydrostatic & {$[-]$} \\
\hline$l$ & Liquid & {$[-]$} \\
\hline$l v$ & Liquid vapour interface & {$[-]$} \\
\hline $\max$ & Maximum & {$[-]$} \\
\hline peak & Thin-film property peak value & {$[-]$} \\
\hline ref & Reference & {$[-]$} \\
\hline$s$ & Wall slip & {$[-]$} \\
\hline$t$ & Derivative in dimensional time & {$[-]$} \\
\hline$\tau$ & Derivative in dimensionless time & {$[-]$} \\
\hline$v$ & Vapour & {$[-]$} \\
\hline
\end{tabular}




\begin{tabular}{|c|c|}
\hline$v_{e q}$ & Vapour equilibrium \\
\hline$v r$ & Vapour recoil \\
\hline vis & Viscous drag \\
\hline$w$ & Wall \\
\hline$x$ & Derivative in dimensional $\mathrm{x}$ \\
\hline$X$ & Derivative in dimensionless $\mathrm{X}$ \\
\hline$y$ & Derivative in dimensional y \\
\hline$Y$ & Derivative in dimensionless $\mathrm{Y}$ \\
\hline
\end{tabular}




\section{Chapter 1}

\section{Introduction}

Technological advances have led to increases in system power while decreasing operational size. This trajectory has resulted in ever increasing power densities and has been paralleled with a growing need to remove waste heat. The methods of removing waste heat have transitioned from singular methods of heat transfer such as convection, conduction and phase change to a combination of these methods. To aid in the removal of waste heat, phase change technologies such as heat pipes allow for the quick and efficient removal of heat from high intensity heat sources such as central processing units or power converters.

The phase change process is facilitated at the liquid vapour interface and is critical to the cooling of high power density systems. The heat is typically conducted and/or convected in the liquid phase to the liquid vapour interface where the heat is removed with the change in phase. Simplified analyses of the phase change process assume the interface to be idealistically flat and uniform; however in industrial applications the interface can take a variety of shapes the most generalised of which being curved. Curved evaporating interfaces are common in grooved evaporators, heat pipes and nucleate boiling, as illustrated in Figure 1. 


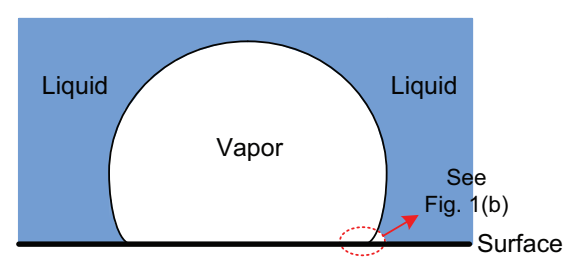

(a)

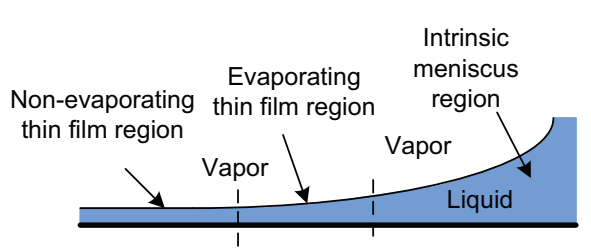

(b)

Figure 1: A nucleate bubble forming on the bottom of a large reservoir [1].

The generalisation of evaporation from interfaces extends the study of liquid vapour interfaces to include bubbles, menisci, curved thin films and flat films, all of which are used in a variety of phase change technologies. In some applications, small structures are implemented so as to facilitate liquid pumping through capillary action. To better design and utilise such cooling technologies, a solid understanding of the principles governing the statics, dynamics and stability of evaporating curved interfaces is needed.

\section{$1.1 \quad$ Research motivation}

Wherever high power density systems are in use, it is likely that phase change heat transfer devices are being used to move and distribute the heat. The physics that govern the statics, dynamics and stability of evaporating curved interfaces are not well understood and have become a growing field of interest in accordance with the growing range of phase change heat transfer applications. As industrial applications continue to push the limits of phase change heat transfer, the point of meniscus transients and instability becomes a point of concern.

The failure of a heat pipe in a laptop can often be fixed quickly and at minimal cost, however the same cannot be said for satellites or aircraft borne radars. Current and future needs are requiring a greater degree of reliability and durability for phase change heat transfer systems such as those used on the Mars Science Laboratory 


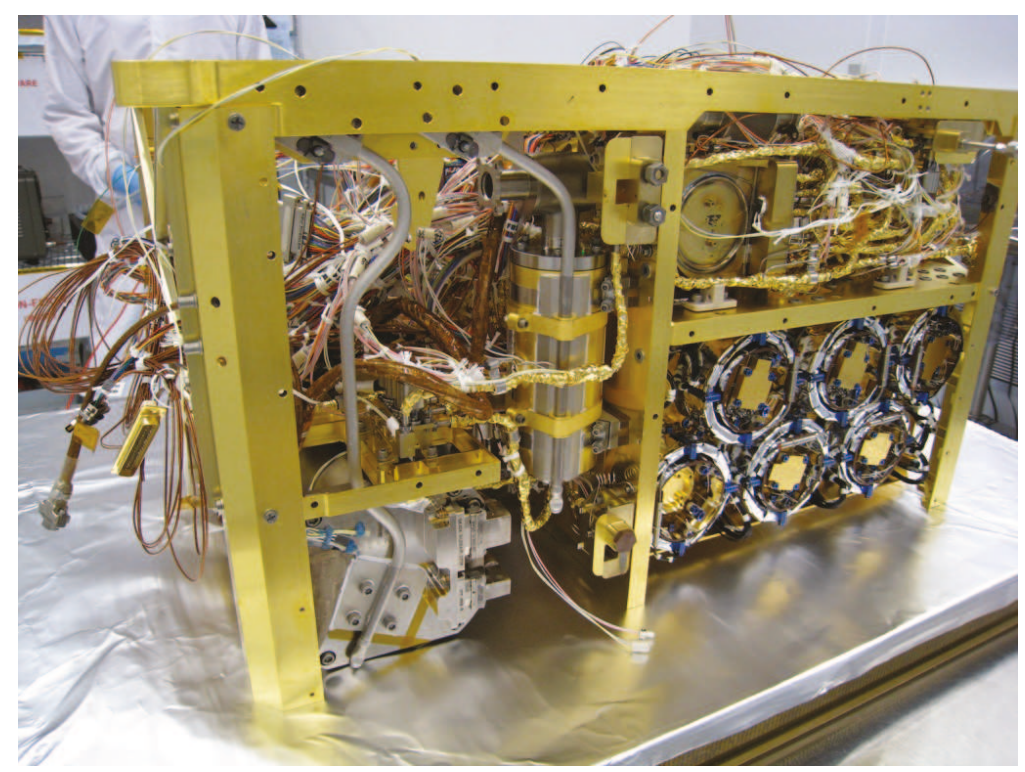

Figure 2: A set of heat pipes on the Sample Analysis at Mars (SAM) payload for the Curiosity mission [2].

spacecraft Curiosity as shown in Figure 2.

While many systems are designed around a steady state operating condition, there exists a potential for the system to undergo some degree of transients and potential system instability. The interface's response to external influences can result in a dynamic response and in certain cases, lead to a runaway condition and rupture of the interface. Instabilities leading to film rupture can cause a dynamic response of the liquid vapour interface that may constitute a failure of the heat transfer device. Thus, to appropriately design and implement two-phase heat transfer devices it is important to characterise these instabilities.

The stability of an evaporating liquid film is governed by a variety of mechanisms spanning: evaporation, van der Waals forces, surface tension, viscous forces and associated secondary effects. The combined interactions are believed to be the basis from which instabilities may arise. The geometric constraints leading to the formation of curved thin films serve to further complicate the problem. 
The potential instability of an evaporating meniscus may be driven by the rupture of the liquid vapour interface upon the substrate. To better describe this instability, let us assume a small disturbance upon the interface results in a surface depression causing a departure from equilibrium as illustrated in Figure 3.

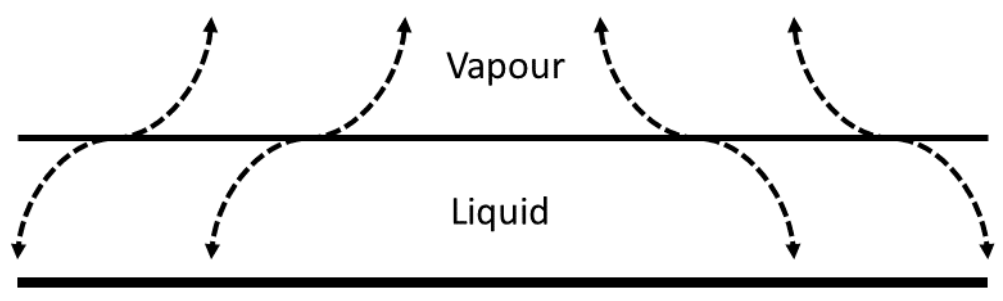

\section{Substrate}

(a) Undisturbed liquid film.

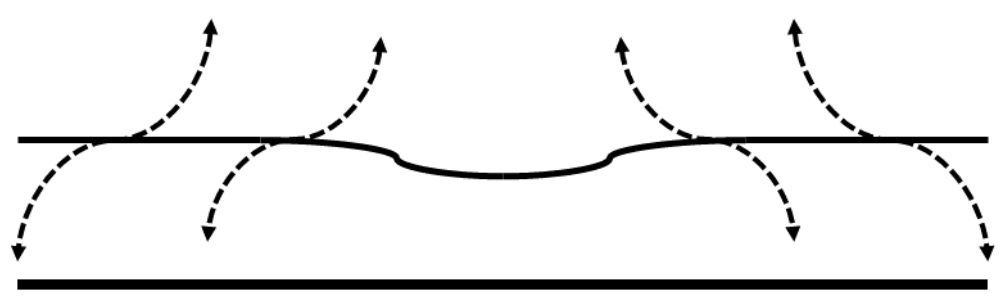

(b) Perturbed liquid film.

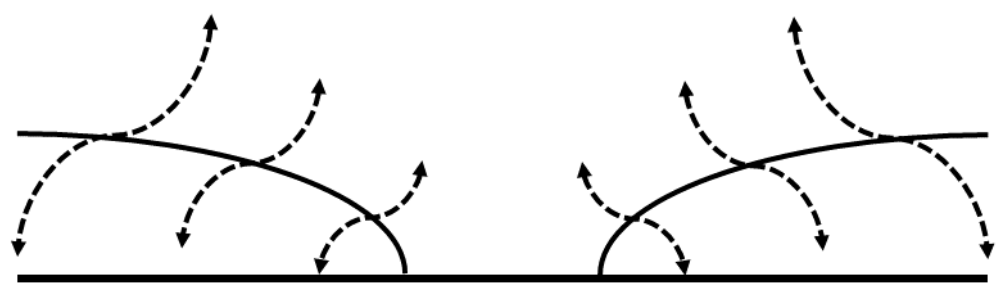

(c) Ruptured liquid film.

Figure 3: The stages of liquid film rupture.

The depressed interfacial region becomes warmer and experiences a local increase in evaporation and by extension vapour recoil. The combination then forces the 
liquid towards the wall, thereby amplifying the disturbance. The changes in interface thickness lead to spatial temperature gradients and in turn thermocapillarity by way of surface tension variation (Marangoni instability). The thermocapillarity serves to further enhance the runaway effect as warmer liquid from the depression is drawn toward the cooler regions of the interface. As the film gets thinner, the increasing disjoining pressure will also provide positive feedback.

Combating these effects is the capillary force as it increases with increasing interface deformation. It is then possible to reach a new equilibrium state by means of rolling cell structures with a wavy interface in a similar fashion observed for fluid instabilities such as Rayleigh-Bénard or Bénard-Marangoni cells. Additionally, localised cooling of the depressed region due to latent heat transfer may reduce the vapour recoil and thermocapillary effects. Such complex interplay among these various mechanisms requires an in depth study so as to accurately characterise the potential for meniscus and thin-film instability and any related interface dynamics. 


\section{Chapter 2}

\section{Literature survey}

Despite the wide array of technological applications reliant upon phase change heat transfer, a limited number of researchers have focused on meniscus based heat transfer. Of the works focused on evaporating menisci, researchers have made advances through theoretical and experimental studies that have shed light on the problem. The following outlines the current level of knowledge regarding evaporating meniscus dynamics, evaporating thin-films and film stability.

\subsection{The interface}

An interface can be described as a region where two or more distinct phases meet. For a single species system comprised of two phases separated by a single uniform interface, the interface can be characterised in two different ways:

\section{Sharp Interface}

A sharp interface is characterised by a discontinuity in the intensive properties of the system. Typically a jump in properties at a discrete point is assumed, implying a binary type of condition at the interface.

\section{Continuum Interface}

A continuum interface requires the intensive properties to be continuous across 
the interface. The intensive property profile exhibits a large gradient over a very narrow region.

In either case, the interface location is averaged in time, as the interface is in a constant state of evaporation and condensation. Typically the time averaging must be sufficiently small so as to not infringe on the transient aspects of the surface's geometry, such as in the case of gravity waves.

\subsubsection{The meniscus}

The meniscus is a specific type of interface condition where the interface is curved. The curvature may be either positive or negative depending upon the intermolecular force interaction between the rigid substrate and the liquid. This force interaction is a function of the fluid's proximity to the substrate. Thus, for large systems, the intermolecular force interaction is too weak to affect the interface geometry.

The meniscus is assumed to be a smooth and continuous interface, formed by the proximity of the fluid and the substrate(s), which produce a symmetry condition at the centre of the capillary geometry. The concavity of the interface is strictly a function of the intermolecular interactions between the substrate and the liquid. If the liquid has a repulsive interaction with the substrate, such is the case with mercury and glass, the meniscus will be convex. However, if the fluid possesses an attractive interaction with the substrate, the surface will be concave, such as the case with water and glass. For the purposes of this study, the meniscus is considered to be concave.

As the meniscus is formed by the solid-liquid interaction, it can be deconstructed into regions of dominating interactions. The principle force interactions within the meniscus can be separated into three regions: the adsorbed region (non-evaporating thin-film), the thin-film region and the intrinsic meniscus region [1,3-6]. The three regions of the meniscus are shown schematically in Figure 4. 


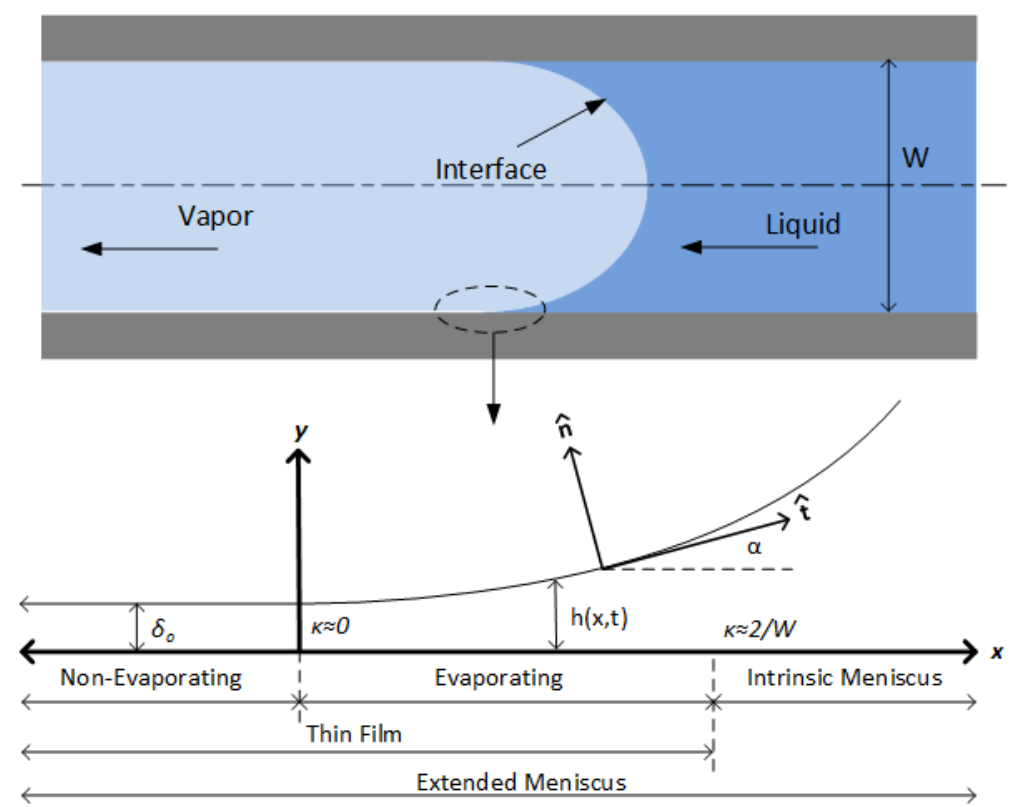

Figure 4: Channel based meniscus and thin film region.

\section{Adsorbed region}

The adsorbed region, also referred to as the non-evaporating thin-film region, is considered to be the portion of a meniscus in direct contact with the rigid substrate. The adsorbed region is dominated by the intermolecular attractive force, resulting in the physical adsorption of the liquid to the substrate [7]. This adsorption principle was applied to thin-film and meniscus studies by Derjaguin et al. [8], and referred to as disjoining pressure. In effect, the intermolecular forces acting on the adsorbed region are averaged over the affected area so as to achieve a pressure relation, Equation (1), which can be used in continuum analyses.

$$
P_{d}=\frac{A}{h^{3}}
$$

For the purposes of evaporating thin-film analysis, the disjoining pressure acting on the liquid serves to hold it in place. This retention of the liquid molecules is assumed to be of sufficient strength to suppress evaporation in the adsorbed region $[7,9,10]$. 
With a lack of evaporation, the adsorbed film is considered to be of a uniform thickness and extends for an unspecified length [6,9-13]. If the substrate is planar in nature, then by extension, the adsorbed film would also be planar.

Using the conditions of no evaporation and no curvature, researchers have extended these boundary conditions such that the capillary pressure in the film is zero $[9,14]$. Furthermore, as the disjoining pressure retains the liquid, it can be further assumed that no fluid flow occurs in the adsorbed region. This then leads to the conclusion that the liquid pressure gradient must be zero throughout the adsorbed region.

These assumptions for the adsorbed region allow for the determination of initial conditions by inspection. As the film cannot evaporate, the mass flux balance for the adsorbed region has a net mass exchange of zero, despite being subjected to an applied superheat $[7,10,12,15]$. Thus it may be inferred that the principal mode of heat transfer is conduction through the adsorbed layer $[7,12,15]$.

Additionally, as the adsorbed layer is assumed to be sufficiently thin (molecular scales), the interface temperature between the liquid and vapour may be assumed to reflect the temperature of the substrate $[7,12,15]$. However, some research has suggested that the contact resistance between the liquid-solid interface, or Kapitza resistance, may produce an interfacial temperature lower than that of the wall temperature $[15,16]$.

Using the aforementioned conditions and restrictions for the adsorbed region, it is possible to determine the thickness of the film for a given set of parameters as done in various works $[7,13,15]$. Though the adsorbed region has been well bounded and is explicit for the assumptions made, some researchers continue to assign arbitrary values of adsorbed layer thickness $[4,15,17,18]$. 


\section{Evaporating thin-film region}

The evaporating thin-film portion of a meniscus forms the transition between the adsorbed region and the intrinsic region of a meniscus. This transitioning from one region to the next, places bounds on the conditions expected for the evaporating thin-film. As such, the region adjacent to the adsorbed region is expected to have a curvature near zero, with zero being the exact location of the transition $[7,15]$. As such, the early portion of the thin-film region is subject to the same intermolecular forces which dominate the adsorbed region. The intermolecular contribution is presumed to be of sufficient strength to make a significant contribution to the interface formation in the early portions of the thin-film.

Idealistically the thin-film seamlessly transitions from the adsorbed region into the evaporating thin-film region. Though logical in its conception, current mathematical models of evaporating thin-films require a set of perturbations at the transition position to avoid a trivial solution yielding a continued adsorbed non-evaporating region [7]. The required perturbations are applied to the interface geometry which include the film's thickness, slope, and higher order spatial derivatives.

From the expected scales of the adsorbed region, the evaporating thin-film region is assumed to be of sufficient thickness so as to permit the application of continuum mechanics $[6,10,19-21]$. Though the current use of continuum models is pushing the limits of applicability for thin-films in general, it has been observed that fluids as thin as $15 \mathrm{~nm}$ still behave like continuous mediums [22]. Though the method of continuum mechanics is widely accepted, other works have probed the thin-film region with the use of molecular dynamics based simulations [1,16]. In these molecular dynamics simulations, domain properties such as negative liquid pressure have been predicted to occur in the thin-film, as is consistent with continuum model predictions $[1,15,23]$.

As the thin-film increases in thickness, the disjoining pressure effects decrease 
in strength as a reciprocal of the film thickness and allow for the film to undergo evaporation. With the presence of evaporation in the thin-film region, some works suggest the potential for local evaporative cooling [3,24-26], while other models are unable to capture this phenomena [14,23, 27-29]. Any local changes in temperature may result in changes to the substrate temperature profile, an aspect typically treated as a constant wall temperature in many models [9, 12, 13, 23, 29]. Such constant substrate temperature models limit the thermal changes in the thin-film region to that solely of conductive losses across the thickness of the thin-film [7, 23, 29]. The conductive losses observed in numerical studies suggest that the thermal losses are very small in the thin-film region [15].

As the thin-film region continues from the adsorbed region, it transitions to the intrinsic region where a constant curvature boundary condition is imposed. The constant curvature condition is dictated by the geometry of the bounding substrate and the fluid's surface tension. To satisfy the intrinsic region boundary condition, the thin-film region grows in thickness and becomes curved. This curving of the thinfilm requires the presence of a capillary pressure. Thus the thin-film will undergo a curvature transition from that of zero to a non-zero constant curvature at the threshold of the intrinsic region. Therefore, the thin-film region is subject to some significant gradients in curvature and by extension, capillary pressure. Such curvature gradients have been measured experimentally [24,30], from which researchers have observed an overshoot in the curvature, followed by a decay to an asymptotic value consistent with that of the intrinsic region.

\section{Intrinsic region}

The last and most prominent portion of a meniscus is the intrinsic region. The majority of a meniscus is comprised of this region, which is the only visible portion of the meniscus to the unaided eye. The intrinsic region is dominated by the capillary 
effects, as the disjoining pressure is considered to be negligible $[7,15,17,23,29]$. This region is considered to be of a constant curvature, $\kappa=2 / R$ for a tube, or $\kappa=1 / W$ for an infinite channel.

This constant curvature condition associated with the intrinsic meniscus was originally described by the Young-Laplace equation [31]. The constant curvature condition implies a symmetry condition at the centreline position between the substrate walls. Thus, the constant curvature condition is typically used by many researchers as the end of the thin-film, and serves as a termination condition for numerical solvers $[7,15,17,24]$.

The conditions of the intrinsic region traditionally used the Young-Laplace equation, which requires a steady state condition and thermodynamic equilibrium [31]. Although this may be valid for some special thermodynamic cases, it is unclear if this is the case in evaporating thin-film studies. These conditions have been partially investigated in some works $[23,29]$, where the numerical results suggest some evaporation occurs in the intrinsic region, though tending asymptotically to zero [6, 10, 21, 23, 29,32].

\subsection{Thin-film model development}

Thin-film models began with the use of geometric observations such as the contact angle made between the liquid and substrate as illustrated in Figure 5. As it can be seen, the thin-film approaches the substrate, curls up along it, and finally comes to a point or end. Visually, an angle can be constructed such that the end of the thin-film forms the base from which a line can be drawn tangent to the surface. 


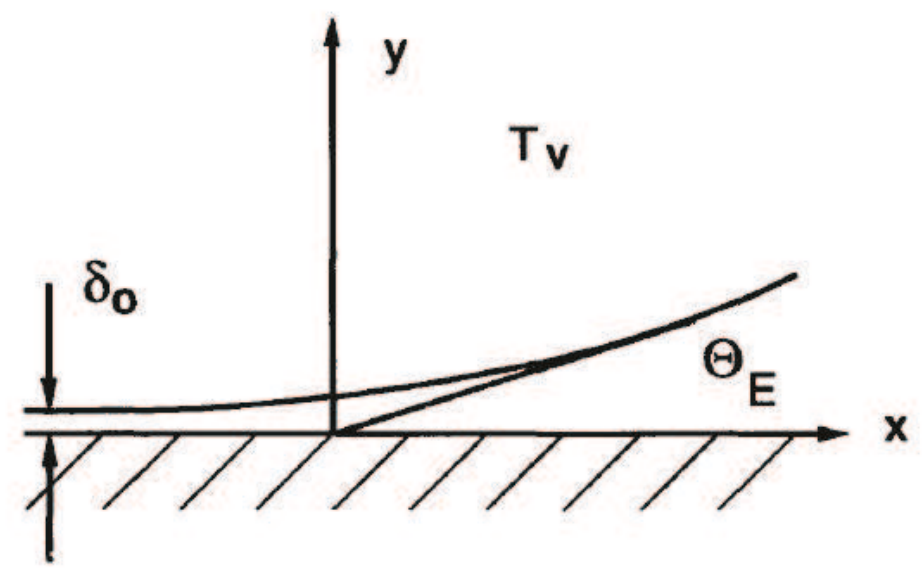

Figure 5: Schematic illustrating the contact angle for an early thin-film analysis [19].

This technique was employed in early works $[11,14,19]$, where the interface could be physically observed and quantised by the instruments of the time. Though this technique provides some qualitative and quantitative information, researchers began to consider the consequences of the method of contact angles [6]. One most notable contribution came about when the question was asked as to what happened when the resolution of the end region was sufficiently small. In short, it was understood that the contact angle would tend to zero nearest the adsorbed region leading to the subsequent failure of contact angle models.

This postulate created a need for a consistent formulation of thin-film models to reflect the physics. Thus an effort to use continuum mechanics in the study of thin-films led to the models now commonly found in literature. These new thin-film models made harmonious use of the Navier-Stokes, continuity, asymptotic methods and Young-Laplace equations to form well rounded models. A significant portion of the models employ the Young-Laplace equation as a boundary condition for defining the end point of a thin-film [7, 15, 17, 23, 24, 29, 33]. 


\subsubsection{Augmented Young-Laplace model}

It is widely accepted that the intrinsic region of a meniscus is governed by the YoungLaplace equation [31]. The Young-Laplace model provides a relation between the curvature of an interface and the associated pressure jump across it [31]. The YoungLaplace formulation as given by Equation (2), requires a thermodynamic equilibrium, no gravity and a steady state condition thus yielding [31]:

$$
P_{c}=P_{v}-P_{l}
$$

The pressure difference across a curved interface is commonly referred to as capillary pressure and can be quantified by relating the geometric conditions of the substrate and the fluid's surface tension. Thus, the capillary pressure is taken to be of the form:

$$
P_{c}=\sigma_{l v} \kappa
$$

This formulation governs the whole of a meniscus in static equilibrium. The YoungLaplace formulation assumed the system to be in thermodynamic equilibrium thereby leading to a hemispherical interface. The original formulation for capillary pressure did not address the intermolecular interactions in the region of the meniscus nearest

to the wall. As such, Wayner Jr. et al. [18] and Schonberg et al. [19] developed a modified form of Young-Laplace equation as:

$$
P_{c}=P_{v}-P_{l}-P_{d}
$$

The augmented Young-Laplace formulation, Equation (4), can accommodate both evaporation and intermolecular effects. The simplified case assumed the interfacial curvature to be hemispherical in nature, however the inclusion of the intermolecular 
forces will result in changes in the interfacial profile. Furthermore, the inclusion of evaporation will further alter the interfacial curvature taking it away from the equilibrium hemispherical shape. This augmented formulation provided a means of investigating evaporating curved interfaces and evaporating thin-films.

Using the augmented Young-Laplace model, researchers have developed a host of models describing curved evaporating thin-films. The models are typically non-linear in nature and require the use of numerical solving techniques. The initialisation of the models can be performed using experimental data as demonstrated by Panchamgam et al. [24]. Though the augmented Young-Laplace model addressed the evaporation and intermolecular contributions, a means of describing the phase change process was required.

\subsection{Evaporation}

While significant effort has been put into understanding phase change, its complete comprehension remains out of reach. One such effort to describe the phase change process is the application of kinetic theory at the liquid-vapour interface. Though kinetic theory based evaporation models are abundant in literature [6, 19, 28, 32, 34], not all results have been experimentally backed and have been challenged in some cases [35-37].

\subsubsection{The Kelvin-Clapeyron model}

The evaporation model most commonly found in the study of evaporating thin-films is the Kelvin-Clapeyron model as implemented by Wayner Jr. et al. [18]. The proposed evaporation model put forth by Schrage [38], was among the earliest to relate the interfacial mass flux to the jump conditions found at the interface, and formed the 
ground work for Wayner Jr. et al. [18].

$$
\frac{d m}{d t}=\left(\frac{2 C}{2-C}\right)\left(\frac{M}{2 \pi R}\right)^{1 / 2}\left(\frac{P_{l v}}{T_{l v}^{1 / 2}}-\frac{P_{v}}{T_{v}^{1 / 2}}\right)
$$

Extending this model further was the work of Wayner Jr. et al. [18] and Wayner Jr. [39], where the Kelvin effect of pressure jump was applied as well as the Clapeyron effect of temperature jump in addition to the following assumptions $[15,39,40]$ :

$$
\begin{gathered}
T_{l v} \approx T_{v} \\
P_{v_{e q}} \approx P_{v}
\end{gathered}
$$

Applying the assumptions of Equations (6) and (7), the Kelvin-Clapeyron model describes an interfacial mass flux as a function of the observed jumped conditions at a liquid vapour interface [39]. A rearrangement and collection of terms reduces the Kelvin-Clapyeron model to the form:

$$
j=a\left(T_{l v}-T_{v}\right)-b\left(P_{d}-P_{c}\right)
$$

Where,

$$
\begin{gathered}
a=\left(\frac{C^{2} M}{2 \pi R T_{l v}}\right)^{1 / 2}\left(\frac{P_{v} M h_{f g}}{R T_{v} T_{l v}}\right) \\
b=\left(\frac{C^{2} M}{2 \pi R T_{l v}}\right)^{1 / 2}\left(\frac{P_{v} V_{l}}{R T_{l v}}\right)
\end{gathered}
$$

The Kelvin-Clapeyron evaporation model has been widely used in literature [19,21, 32, 39, 40], though limitations exist as discussed by Wang et al. [15]. 


\section{$2.4 \quad$ Numerical studies}

The basic understanding and inferences of evaporating thin-films as outlined previously, have allowed for the numerical investigation of the various models proposed. The methods and techniques used to model and simulate evaporating thin-films are typically continuum-based mathematical models, computational fluid dynamics and molecular dynamics. Of the three methods available, the method of continuum based ordinary differential equations is favoured as it offers greater control while potentially providing valuable insights. Though the equations involved are beyond analytical methods, numerical integration techniques such as Runge-Kutta have made it possible to solve the governing equations.

\subsubsection{Continuum models}

For the studies where Ordinary Differential Equations (ODEs) are used in the development of an evaporating thin-film model, the governing equations have been traditionally solved using Runge-Kutta, finite element methods or Adams method. The Runge-Kutta method of solving the governing ODEs has been used extensively with good results [10, 26, 41-43].

Using the augmented Young-Laplace equation to develop a thin-film model, the governing equation can be obtained. Typically the governing equation(s) are classed as second, third or fourth order ODEs $[10,14,26,41,42]$. The principal difference in the order of the governing equation is based on the decision of the researcher. That is to say that the fourth order differential equations are in fact the third order equation differentiated once more with respect to the film length [29]. The rationale behind the use of this superfluous differentiation is so that the fourth order differential equation does not contain an integral term [15]. This differentiation is in some ways beneficial as the solving of the governing equation no longer requires the film thickness or surface 
temperature a priori. When solving the governing equation(s) with a Runge-Kutta integration technique, a set of perturbations are required on the geometric initial boundary conditions so as to avoid a trivial solution [7].

The perturbations required to obtain a non-trivial solution require the use of a shooting technique $[7,15,18]$. Some of the perturbations and their associated effects were discussed in $[7,10,23,29]$. In all of the cases reviewed their appeared to be no correlation between the magnitude of the perturbations and the geometry or applied superheat $[7,15,18]$. Furthermore, no research work has provided a means of consistently perturbing the initial conditions. Aside from the traditional numerical integration and shooting methods used, Panchamgam et al. [24], used experimental data to formulate a solution. Though this is a significant stride forward in the validation process, it is still insufficient in the objective of predictive modelling.

\subsubsection{Behavioural characteristics}

The main observation found in all studies indicates that the governing differential equation is sensitive to specific perturbations, which in turn are a function of the equation order. For the case of the fourth order differential equation, Wang et al. [13, 15] stated the solution was found to be sensitive to the second derivative. In the third order model, the sensitivity was found to reside in the first derivative [7, 10, 23, 29].

Subsequent sensitivity analysis performed by Ball [23] noted the required perturbation to be related to the applied superheat. Further work demonstrated a correlation between the slope perturbation and the accommodation coefficient as illustrated in Figure 6. Thus, Ball [23] was able to confirm the existence of an interdependence of the perturbation with respect to typically set parameters in thin-film models. 

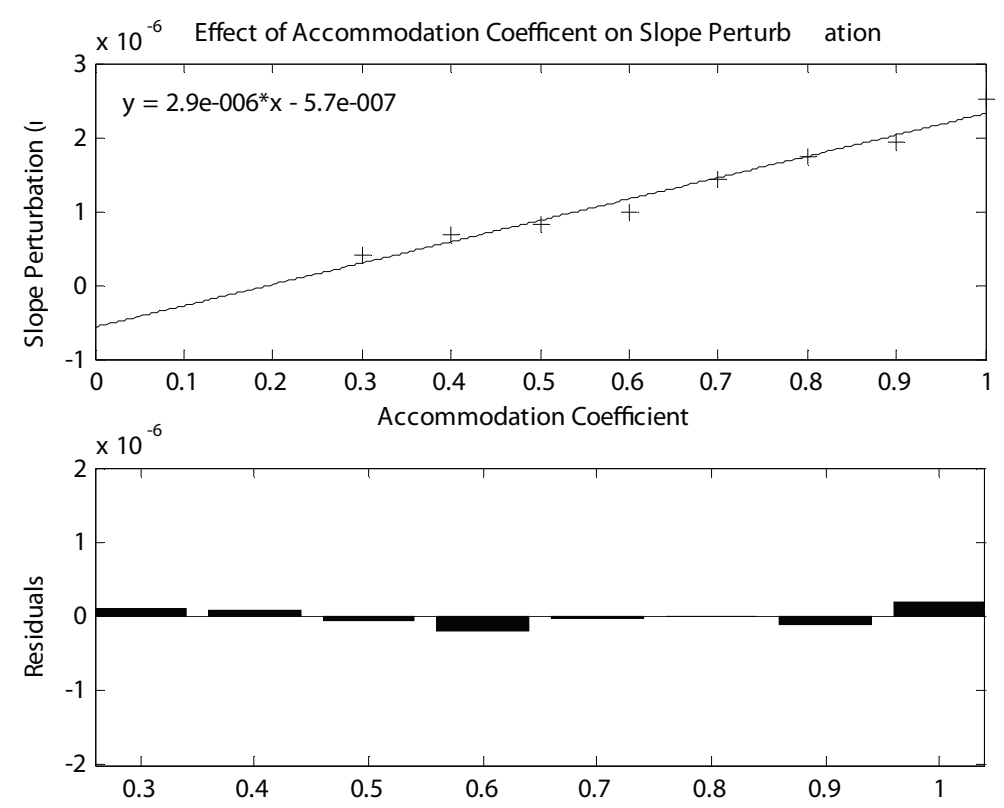

Figure 6: Perturbation and accommodation coefficient relation [23].

As to the natural tendencies of the governing equation, it has been observed in the works of Polansky [29] and Ball [23], that the governing equation tends toward an asymptotic curvature in the far field region of the solution. This is in close agreement with the assumption that the curvature in the intrinsic region is constant. In addition to the far field curvature condition, the curvature was found in many cases to exhibit an overshoot which is confirmed in the experimental observations of Panchamgam et al. [24]. Finally, uniqueness of the solution to the governing equation was investigated by Polansky [29], to which it was concluded that there exists a range of solutions to the governing equation for a given set of conditions.

\subsection{Alternate models}

Alternatively, some researchers have elected to study thin-films using molecular dynamics and computational fluid dynamics (CFD) simulations. Such approaches have 
been used to perform two and three dimensional studies. Others have opted to investigate the transience of the thin-film with varying thermal conditions.

\subsubsection{Computational fluid dynamics}

Thin-film studies using CFD have been principally oriented toward the study of the intrinsic portion of a meniscus bounded by a substrate structure [20,28,44,45]. Though limited to the intrinsic region of a meniscus, CFD models allow for the study of complex geometries such as spheres, cylinders, tubes and channels. Of the simulations conducted, insights have been gained regarding the liquid and vapour flow fields, temperature and evaporative mass flux. The results of such studies make it possible to refine mathematical models and improve their predictability.

A study of the meniscus intrinsic region by Wang et al. [20] was able to confirm that body force effects on flow are related to the diameter of the capillary tube as illustrated in Figure 7. As can be seen in Figure 7, the flow can shift from that of an axisymmetric flow, to that of a skewed recirculating flow field. 

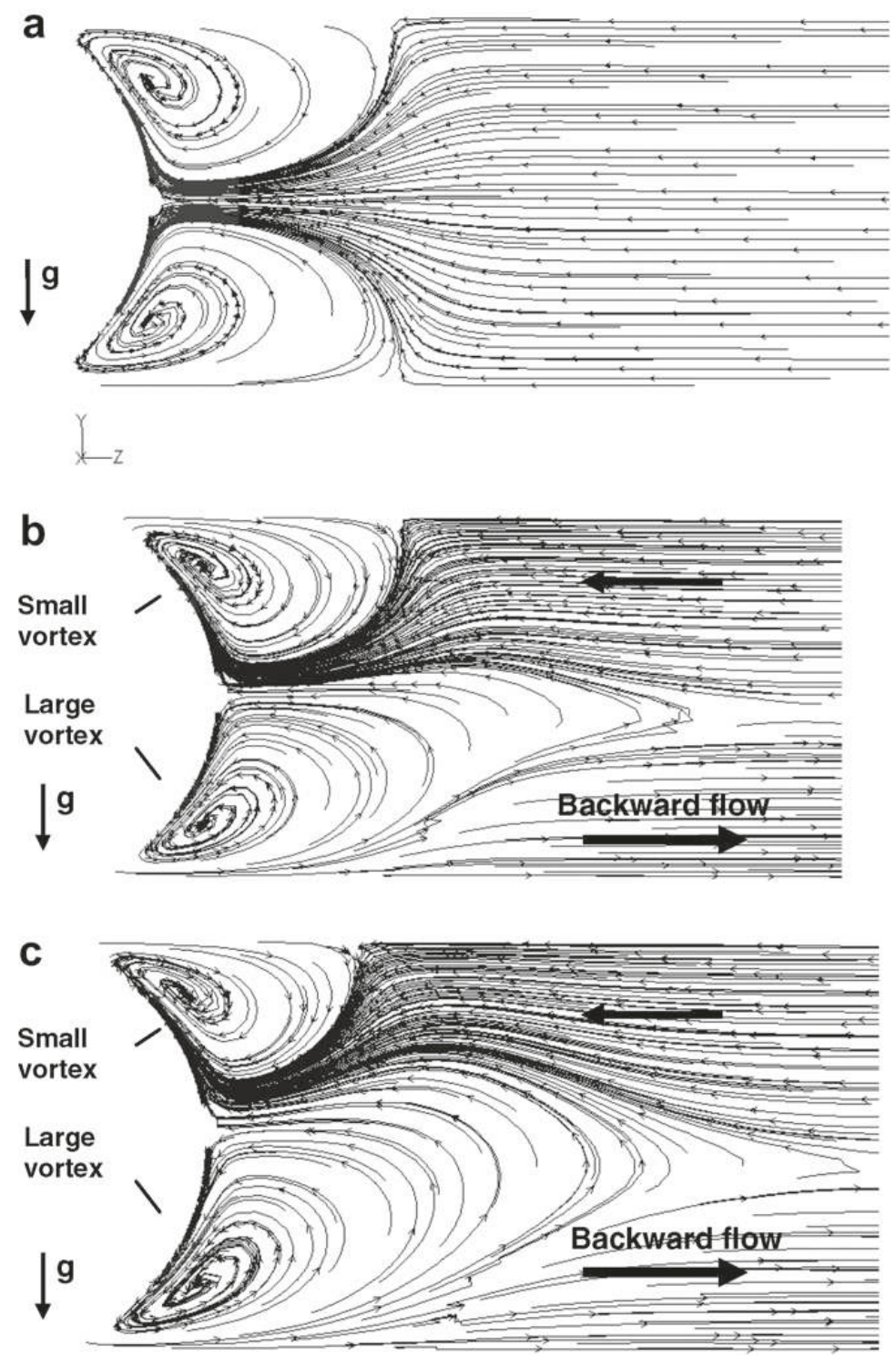

Figure 7: CFD analysis of capillary tubes in a gravitational field [20].

The existence of a recirculating condition below the intrinsic meniscus has been theoretically noted for in other works [28,44-46], and experimentally validated through qualitative analysis by Wang et al. [20]. Further work by Dhavaleswarapu et al. [46], simulated a skewed channel configuration in hopes of obtaining more information regarding the flow near the thin-film region. The domain used was only that of 
the intrinsic meniscus region as indicated by numerical models [10,14, 23, 29,32], and experimental studies $[12,24]$. Other efforts have attempted to use the Young-Laplace condition to model the interface geometry [28,45], a contact angle geometry [20,45] or experimental data [46] to obtain the interface geometry and better describe the CFD domain. Despite capturing the intrinsic region recirculation, no CFD model has been able to accurately model the thin-film region.

\subsubsection{Molecular dynamics}

As an evaporating meniscus spans the scales of macroscopic through microscopic, the modelling of such a region must also span the macroscopic through intermolecular force contributions. As such, molecular dynamics and intermolecular forces are required to analyse the evaporating thin-film. Though many works have been founded in continuum mechanics $[6,14,19,32,47]$, some researchers have opted to investigate thin-films using molecular dynamics based simulations. These works have focused on the existence of adsorbed layers, fluid velocity, accommodation coefficients, Hamaker constants and rates of evaporation.

The work of Maroo et al. [1], studied a channel based evaporating meniscus in which they confirmed the evaporation rate was highest in the thinnest portion of the thin-film and an adsorbed layer was present. Their analysis indicated the averaged surface temperature increased in a smooth manner when transitioning from the intrinsic to the thin-film region. A study of the liquid pressure in the meniscus revealed the presence of absolute negative liquid pressure [1]. This phenomenon of absolute negative liquid pressure has been experimentally confirmed in other works outside thin-film studies [48-53].

Adding to the continuum models, molecular dynamics simulations have provided information regarding the solid-liquid interaction. These simulations suggest the potential for a wall velocity or slip [26], as well as the existence of a thermal resistance 
known as the Kapitza resistance [16]. The notion of wall slip is not particularly new and has been implemented in various mathematical models, while the temperature jump at the solid-liquid surface has not.

Lastly, some researchers have used molecular dynamics simulations to model the process of evaporation and/or condensation at an interface [54-56]. These models are important as they provide insight into the evaporation and condensation coefficients used in mathematical models. The simulations performed by $\mathrm{Yu}$ et al. [55], were able to statistically determine the accommodation coefficient for Argon. This is complemented by works of Tsuruta et al. [56] and Matsumoto et al. [54], who extended the previous work and found stark differences in the evaporation and condensation coefficients for varying fluids. In the limited number of available studies on molecular evaporation dynamics, these findings have not been implemented in continuum models.

\subsection{Experimental investigations}

While the theoretical study of thin-films has a rich and diverse history, it requires validation in order to develop. Few researchers have attempted to experimentally study the evaporating meniscus as the measurement options are severely limited by the scale of the problem. Current measurement techniques have been limited to geometry, temperature and velocity. These measurements are taken by optical means, with the data being obtained from a single image and meant to be representative of steady state.

\subsubsection{Thin-film geometry}

A set of experiments were performed using a microscope and a CCD camera to capture images of an evaporating meniscus $[24,30,34,57,58]$. The images collected were then 
analysed to yield the shape and profile of an evaporating meniscus. The limiting factors in resolving the thin-film and meniscus included the resolution of the optics and the pixel size of the CCD sensor. The results of the experimental studies confirmed the existence of a curvature overshoot on the interface of an evaporating meniscus [24, 30, 34, 57, 58]. Additional experimental studies by $\mathrm{Qu}$ et al. [12] investigated how pore radius affected the heat flux from an evaporating thin-film. Their results indicated a decrease in the thin-film length with an increase in the applied heating load. Such experimental findings have served to validate the predictions made from the mathematical model developed by Polansky [29] and Ball [23].

\subsubsection{Temperature profile}

The measurement of thin-film temperature is among the most difficult tasks. Höhmann et al. [3] and Sodtke et al. [25] attempted to measure the temperature of the substrate on which the evaporating meniscus was placed. They made use of thermochromic liquid crystals (TLCs) as a means to visually obtain the temperature profile of the substrates surface as illustrated in Figure 8. Using a colour camera and microscope, they were able to obtain two dimensional temperature profiles of the substrate surface just below the liquid-solid surface. 


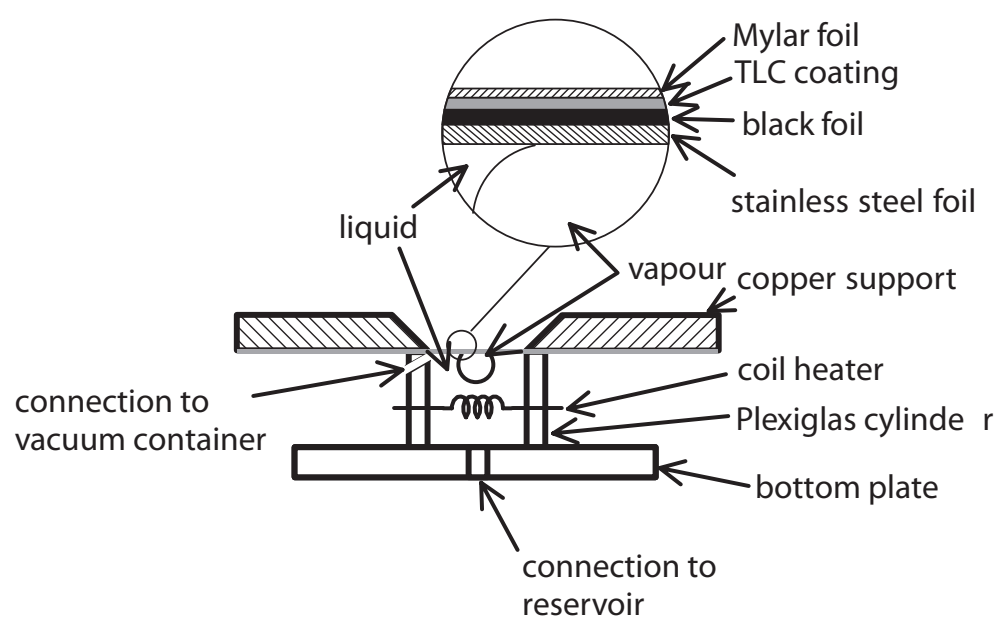

Figure 8: Thermochromic liquid crystal application in thin-film substrate temperature measurement [25].

The experimental results confirmed the existence of localised cooling in the thinfilm region for a channel configuration as well as a single vapour bubble in low gravity conditions. This finding supports the theory that the thin-film region is responsible for a significant portion of the heat flux.

\subsubsection{Velocity profile}

The last and no less complicated experimental measurements are the velocity of the fluid field. The technique of particle image velocimetry (PIV) was used by He et al. [59] and Buffone et al. [27] to map the flow fields in an evaporating meniscus. The particle size was observed by He et al. [59] to produce a diffraction and interference pattern around the particles, similar to that observed by $[24,30,34,57,58]$.

The experiments of He et al. [59] were able to measure the flow field near the contact line region and confirmed the existence of recirculation cells located along the solid substrate and flowing up around and along the free liquid-vapour interface. This recirculation in the intrinsic region of a meniscus was also experimentally confirmed by Buffone et al. [27,60,61]. Using a similar PIV technique, they mapped out the 
vortical structures in the intrinsic region with their work focusing on the conditions of heated and unheated menisci. Their findings show a change in vortex direction for the heated (clockwise) and unheated (anti-clockwise) cases [60]. The reversal of the vortex direction was attributed to a thermally driven surface tension gradient. In addition to this, asymmetric evaporation was observed from the meniscus suggesting the imposition of a symmetry condition at the centreline may not be valid in some cases [61].

This exceptional work has provided some much needed insight into the flow field structure for the intrinsic region of a meniscus. However, as thin-films are still not well studied some experimental techniques are needed to probe this region. Current thinfilm models are based on the assumption of pure substances, thus the introduction of particulates into the flow field will most certainly alter any natural flow characteristics and any data obtained would be biased.

The bias in the data would stem from the presence of the particles and will introduce anomalies such as altered Hamaker constant values or structural disjoining effects. Such structural disjoining effects are noted by Zhang and Lu [22], where the normally free and randomly distributed particles become confined and begin to form a lattice type structure in the liquid field.

\subsection{Meniscus stability}

The sum of the aforementioned research has been focused on the study of steady state thin-film evaporation. Though steady state thin-film study is very important in describing the thin-film and its capacity to transport heat, it is only one aspect of an evaporation meniscus. Instabilities leading to thin-film rupture can cause a dynamic response of the liquid-vapour interface and may constitute a failure of the heat transfer device. 


\subsubsection{Meniscus dynamics}

While much work has been done regarding the statics of a thin-film and meniscus, a supplementary set of studies on meniscus dynamics has been performed. The early works of Lucas [62], Bosanquet [63], Bell and Cameron [64] and Washburn [65] formed the theoretical basis of capillary based meniscus dynamics. The general study of meniscus dynamics has been pursued using theory, numerics and experimentation. Of the studies performed, a variety of cases have been considered such as: liquid-wall slip [65-67], surfactants [68,69], transient contact angles [70-78], gas/vapour displacement $[68,79]$, evaporation/condensation $[47,70,80]$, venacontracta or jet conditions $[78,81-$ 84] and capillary orientation with gravity [85].

The dynamics of a meniscus motion led some researchers to investigate the various regimes of meniscus motion, from which they developed criteria to predict oscillatory behaviour $[84,86,87]$. Fries and Dreyer [88] investigated how the competing forces exchanged dominance through the course of a meniscus rise. This work was succeeded by a systematic analysis of the non-dimensional governing equations describing imbibition [89]. The majority of the studies focused on tube based meniscus dynamics and Newtonian fluids. Levine et al. [67] expanded the study of meniscus dynamics to include channel based imbibition, while Kornev and Neimark [90] introduced viscoelastic fluids.

To complement the work, Siebold et al. [71] experimentally confirmed that the meniscus can exhibit changes in curvature during the dynamic portion of the rise. Lorenceau et al. [81], experimental study found that the imbibition process can produce a liquid finger during the initial stages of meniscus rise, further supporting Siebold et al. [71] observations.

During meniscus rise, the vapour contained within the capillary is displaced, which leads to a viscous drag and a retardation of the flow. The viscous pressure drop 
attributed to the displaced vapour experimentally observed by Zhmud et al. [68], and shown to have considerable effect on the dynamics of meniscus rise. The potential for slip at the solid-liquid interface in capillaries was put forward by Washburn [65] and experimental studies by Pit et al. [91] support the potential for slip. Furthermore, slip at the solid-liquid interface as reviewed by Neto et al. [92], can be extended to capillary based flows.

While not principally studying the meniscus dynamics, the experimental study performed by Kuz'mich and Novikova [93] investigated the static height of an evaporating meniscus as a function of capillary radius and capillary tube length. The study made only a qualitative mention as to the dynamic aspect of the meniscus motion. The observed oscillatory motion by Kuz'mich and Novikova [93] was noted to be dependent upon the capillary radius and attributed to changes in the wetting angle. Furthermore, the evaporation condition was a result of changes in ambient vapour pressure and not heating.

\subsubsection{Meniscus stability}

Motion of an evaporating meniscus may or may not be desirable. Depending on the application, the conditions which dictate the onset of such behaviour should be characterised and understood. It may be speculated that a rupture of the thin-film in an evaporating meniscus may cause a shift in the interface and change in the heat transfer properties. Though the dynamics of an evaporating meniscus have been investigated to some extent, the existence of any thermally driven instability mechanism leading to such dynamics remains open. Such conditions are of interest given their potential application to devices such as heat pipes.

In looking for meniscus or thin-film stability, the effects of various types of perturbations can be investigated so as to determine the potential for instability. Should there be a potential for instability, the time and location of rupture may be of some 
interest. The point of rupture is typically described as the point where the film achieves a thickness of zero and the liquid-vapour interface makes contact with the solid substrate [94-96]. As applied to heat pipes, a rupture of the thin-film may cause the meniscus to oscillate, leading to a potential dry out condition and complete failure of the device. As such, the study of evaporating meniscus/thin-film has become a priority with emphasis on searching for the conditions associated with the onset of instability.

\section{Planar film stability}

The study of liquid film stability was founded for the simplified case of planar liquid films; it benefits from a reduction in complexity while still providing insights as to any potential instability mechanisms. The stability of thin liquid planar films is based on the principal that a thin liquid layer rests atop a rigid planar substrate. This problem has been investigated through a series of theoretical studies aimed at describing the film and its propensity to destabilise. The earliest of these studies focused on how long range molecular forces in non-evaporating films could lead to instability [97-99]. The problem was first broached by Sheludko [97] who used a thermodynamic approach to determine how long range molecular forces can lead to instability and the eventual rupture of the film for a given perturbation. The potential for thin liquid film destabilisation was correlated with a characteristic film thickness. The potential for a perturbed flat film to destabilise was further supported with the findings of Vrij [98] and Ruckenstein and Jain [99] who correlated the critical film thickness with a critical perturbation wavenumber.

The study was advanced by Williams and Davis [94] who constructed a non-linear stability model. The non-linear stability model was investigated through numerical analysis using a finite perturbation. The results of the numerical simulation determined that the use of the linear stability theory was insufficient as higher order terms 
had significant effect. The non-linear model was noted to reduce the time to rupture as compared to the linear model in addition to a shift in the required perturbation wavelength. The deviation in rupture time for both linear and non-linear stability theories was found to be as large as an order of magnitude [94]. In addition to the rupture time deviation observed, limitations in the validity of linear stability theory arise as it is only valid for infinitesimal departures from a planar interface [94]. This finding was further supported by Sharma and Ruckenstein [100] who also found the nonlinear effects reduce the rupture time, with model having the addition of Marangoni effects by way of surfactants. The Marangoni effects were noted by Sharma and Ruckestein [100] to enhance the films stability.

Davis [101] continued the study of Marangoni effects on flat film stability with the introduction of thermocapillary effects. This analysis highlighted the thermal gradients as the source of energy for hydrodynamic instabilities, which manifest as two- and three-dimensional surface waves. With thermal gradients comes the potential for phase change as was the focus of Bankhoff's [102] study on evaporating and condensing thin liquid films on an inclined plane. The effects of phase change were noted as destabilising for evaporation and stabilising for condensation.

Combining the aforementioned influences, Burelbach et al. [96] developed a mathematical formulation describing a planar film using a one-sided model. The singlesided model was achieved by assuming that the vapour fluid properties were small by comparison to the liquid properties, as for example in $\rho_{v} / \rho \simeq 0$. This approach has been routinely used in steady state thin-film analyses [7, 12, 15, 18, 24], with only a limited number two sided models $[1,4,5,17]$. The resulting model then had a simplified free surface boundary condition thereby yielding a single non-linear differential equation. Using a perturbation analysis, they were able to determine the potential for thin-film instability [96]. The numerical results of the analysis demonstrated the film was liable to destabilise faster for an evaporating film than for a condensing film. 
The conditions necessary for instability were related to the nature of the perturbation by way of a critical wavenumber as is consistent with previous works [97-99].

Following this work, Shklyaev and Fried [103] developed a modified linear stability model by using a different evaporation model than that used by Burelbach et al. [96]. The model sought to investigate the assumptions of previous works with emphasis on the validity of the lubrication approximation and the evaporation model. The results of Shklyaev and Fried's [103] investigation proved the lubrication approximation as applied to wavy unstable thin-films is violated as the film approaches the point and time of rupture [103]. Furthermore, the evaporation model was determined to affect the thin-film stability via vapour recoil and total pressure, both of which were found to be stabilising in nature [103].

\section{Multi-dimensional spatial stability}

Most thin-film stability analyses have focused principally on the time and location rupture and in so doing, have failed to account for any spatial aspect(s) of thin-film rupture. The multi-dimensional aspect is of great significance to industry as some industrial processes are one-dimensional; this is the case for coatings, optical coatings and paint [104]. In such industrial settings the interaction of two or more spatial directions can result in the interference of surface waves leading to altered positions and times of rupture.

Building upon the planar thin-film stability analyses, Benselama et al. [105] developed a mathematical model describing the stability of an evaporating curved interface. The analysis included contributions from long range molecular forces, surface tension and thermocapillarity effects. The analysis was simplified through a limiting of the evaporation so as to neglect vapour recoil effects. Using a domain perturbation analysis and retaining higher order terms, the curved evaporating thin-film stability was found to be dependent upon the sign of the Marangoni number. Subsequent work by 
Avramenko et al. [106], continued the study of curved thin-film stability with thermocapillarity and long range molecular forces. The analysis demonstrated that changes in the interfacial temperature gradients can shift the location of the peak replenishing fluid velocity and introduce a spatial component to thin-film stability.

The spatial study of interfacial stability was extended to three dimensions by Witelski and Bernoff [104]. The thin-film was modelled by a fourth order partial differential equation. The model was subject to two boundary conditions at the origin and far field portions of the domain. The far field boundary condition was found to be sensitive to the initial conditions, with effects noted to change the time and location of rupture [104]. The model was solved using a shooting method, Newton's method and matched asymptotics to achieve the far field boundary condition [104].

For each set of initial conditions provided, the solution obtained was found to be one among an infinite set of solutions to the governing equation [104]. Thus it was suggested that the solutions and rupture conditions for the governing equation could be represented by Helmholtz eigenvalues [104]. In the analysis, Witelski and Bernoff [104], opted to investigate the energy of the system and use this quantity for measuring the potential onset of instability. A simplification was imposed, such that the thickness of the film was averaged for each analysis and the energy of the total system obtained [104]. The results of the simulations confirmed that for an axisymmetric domain, the film had both uniform and non-uniform solutions just prior to rupture as illustrated in Figure 9. The modes of film rupture were noted as a planar contact line, a singular point on the surface or a combination of both. 


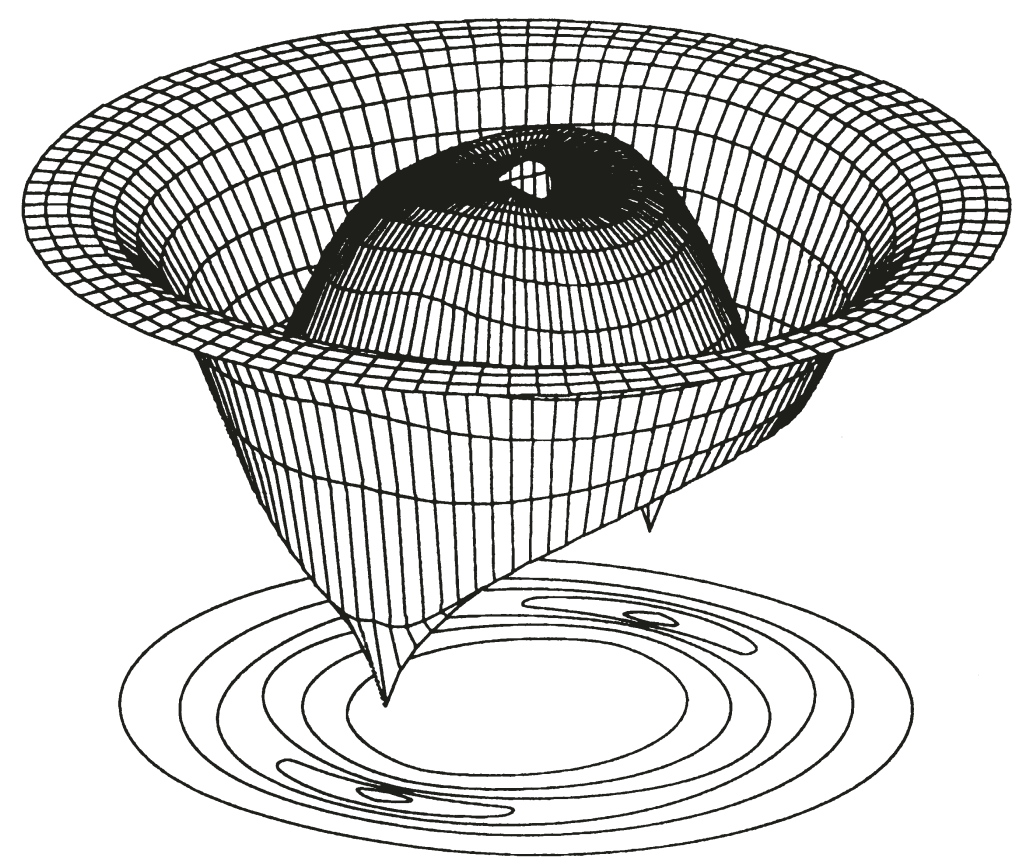

Figure 9: Coupled ring and point rupture modes [104].

\section{Experimental study of stability}

The stability of an evaporating meniscus is a complicated topic as it involves several complex phenomena together: evaporation, long-range molecular and viscous forces, surface tension, vapour recoil, and thermocapillarity. While the principal mechanisms responsible for the meniscus instability have been previously outlined through a variety of theoretical works, the successful demonstration of meniscus instability is another matter. Such experimental evidence has been in short supply as noted in Oron et al. [107] who call for more experimental thin-film research. Since their call for experimental studies, some have been put forth in the general area of thin films [108], with only a couple in the area of evaporating meniscus stability such as Pratt et al. [109] and Harmand et al. [110].

In their experimental study of evaporating menisci, Preiss and Wayner [111], were among the first to observe meniscus instability. The experimental study was principally focused on changes in curvature with increasing evaporation rates. In their 
pursuit of greater changes in meniscus geometry, they briefly reported on the observation of meniscus sputtering followed by an oscillation of the meniscus. These observations of meniscus sputtering and oscillation inspired other researchers to perform experimental studies focused on the instability itself.

Pratt et al. [109] performed two experimental studies with the intent of studying an unstable evaporating meniscus. The two different experiments were that of a heated cylindrical capillary tube within an atmospherically controlled chamber and a single pore loop heat pipe. Changes in the contact line geometry around the time of destabilisation were captured using a high speed camera in addition to wall temperature measurements above and below the meniscus. These temperature measurements were used to formulate a simplified stability criterion from which to infer the potential for meniscus instability. The stability criterion was developed upon the theoretical framework of Burelbach et al. [96]. This work was later followed up by Garcia [112], from which a modified stability criterion and experimental set were produced.

Further advancement came from the work of Buffone et al. [27], in which they focused on the hydrodynamics and stability of a meniscus subject to a temperature gradient. Using micro PIV in concert with a high speed camera and an infrared camera, they were able to investigate the interface dynamics and changes in the contact angle. Their findings indicate that upon heating, the meniscus has a change in intrinsic flow vorticity direction, contact angle and meniscus height. This work was later continued by Buffone et al. [113] with a closer look at the Marangoni-driven aspect of meniscus instability. Their linear stability model suggested the instability was attributed to a self-induced regional temperature gradient in the thin-film region.

Using a constrained vapour bubble, Plawsky et al. [114] was able to investigate an evaporating thin-film region while oscillating. The thin-film was destabilised with strategically placed heaters and coolers on the constrained vapour bubble. The destabilised film was observed to have some degree of oscillation leading to temporal 
changes in local curvature and thickness. This work was furthered by Harmand et al. [110] with their experimental investigation of a capillary channel based evaporating meniscus. The experiments used a Tantalum coated micro-channel, a programmable pump, high speed camera and infrared camera in an ambient atmospheric environment with ethanol, acetone, FC-72 and water. The unheated stable menisci were observed to have unique and complex shapes that may be attributed to the end effects of the channel. The meniscus was in effect destabilised with increased evaporation rates, with the instability having large scale motions over an extended period of time.

\subsection{Summary}

The study of an evaporating meniscus and by extension thin-films (flat or curved), is critical to the advancement of current heat pipe technology. Many works have targeted the thin-film region theoretically $[3,7,9,15,17,18,24]$, and a few experimentally $[3,20,27,30,59]$. So far, the study of evaporating menisci and thin-films has provided some insights into the processes at play. Though these advances have been illuminating, their application has been limited to static analyses and remains unused in the study of thin-film stability.

This region of transient behaviour has been investigated to some extent theoretically $[47,68,79,80,84,86]$ and to a lesser extent experimentally [69, 78, 81, 86]. Further investigations as to the stability of the meniscus and thin-films have become a more recent point of study theoretically [96-100] and with limited success experimentally $[27,94,109,111,113,114]$. Despite the advances in the study of meniscus dynamics and stability, a significant amount of questions remain. As such, it is the objective of this work to address the following two queries:

1. What aspects govern evaporating meniscus dynamics? 
2. What are the principal factors that affect thin-film stability?

In an effort to address the first query, Chapter 3 will outline a theoretical analysis of evaporating meniscus dynamics followed by an experimental validation in Chapter 4. The second query will be addressed in Chapter 5 with the formulation of a linear stability model describing an thin-film subject to phase change. The theoretical analysis is then complimented with an experimental study consistent with the theoretical formulation in Chapter 6. Finally, a summary of the principal findings is put forth in Chapter 7. 


\section{Chapter 3}

\section{Meniscus dynamics - model}

When a thermal load is applied to a liquid filled capillary the meniscus interface will facilitate the phase change process. This change of phase can be either evaporation or condensation depending on the thermal load. The objective of this work is to develop a theoretical basis describing the flow dynamics and the potential for instability for an evaporating meniscus.

\subsection{The flow domain}

The capillary structure is based on a cylindrical capillary tube of a constant diameter $(D=2 R)$, idealistically straight and of immiscible walls. The tube is assumed to be oriented with the capillary tube's long axis normal to the reservoir interface and gravity acting in the negative $z$ direction. When the capillary tube makes contact with the reservoir's interface, the tube begins to fill and a meniscus is formed as illustrated in Figure 10. 


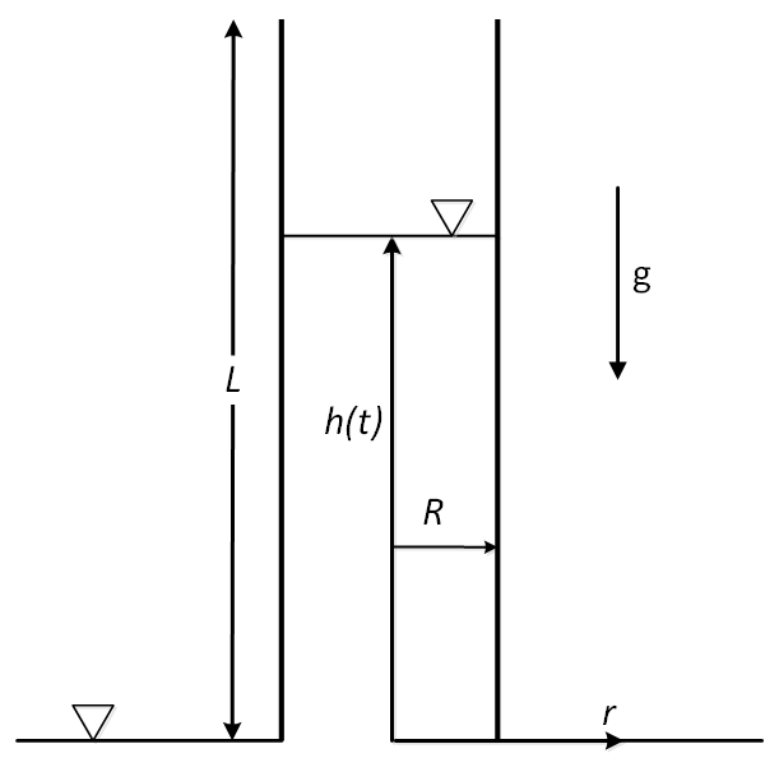

Liquid Reservoir

Figure 10: A cylindrical capillary tube with the associated liquid reservoir and meniscus.

The capillary tube is placed such that the entrance of the tube is at or below the reservoir's free interface. Given the cylindrical nature of the problem, a cylindrical coordinate system is employed with the origin situated at the tube's symmetry axis coincident with the z-axis and the reservoir free interface bisecting the z-axis at $z=0$.

Depending on the flow properties within the capillary tube, there exists a potential for the fluid flow to become turbulent. Under such conditions the flow would undoubtedly become two or three dimensional. Using a Reynolds number analysis, it is assumed that turbulence is expected to occur at or above $R e=2300=O(3)$. Assigning the appropriate orders of magnitude to the fluid properties and geometry, the range of flow velocities required to achieve turbulent flow are in excess of $1 \mathrm{~m} / \mathrm{s}$. Therefore the flow is assumed to be laminar along the long axis of the capillary tube. 


\subsection{Generalised capillary flow}

For the meniscus to move, the liquid column contained within the capillary must move. The liquid flow field and by extension the interface motion can be obtained from Navier-Stokes:

$$
\rho \frac{D \mathbf{V}}{D t}=-\nabla \mathbf{p}+\mu \nabla^{2} \mathbf{V}+F_{b o d y}
$$

The following assumptions are then applied to Equation (11):

1. The flow is unidirectional along the z-axis (laminar)

2. The liquid is incompressible

3. The velocity profile is fully developed

the flow in the capillary is then driven by the pressure gradient as:

$$
\frac{\partial P}{\partial z}=\frac{\mu}{r} \frac{\partial}{\partial r}\left(r \frac{\partial w}{\partial r}\right)
$$

To obtain the flow field in the tube, Equation (12) can be integrated as,

$$
\int r \frac{d P}{d z} d r=\int \mu \frac{d}{d r}\left(r \frac{d w}{d r}\right) d r
$$

which yields,

$$
w=\frac{1}{\mu} \frac{d P}{d z} \frac{r^{2}}{4}+\frac{1}{\mu} C_{1} \ln (r)+C_{2}
$$

The velocity profile within the capillary tube is solved using the following boundary conditions:

$$
\begin{gathered}
r=0, \quad w=\text { finite } \\
r=R+\Delta R, \quad w=0
\end{gathered}
$$

Applying Boundary Condition (15) to Equation (14), the natural logarithm tends 
to negative infinity. As the fluid flow at the centre of the tube is finite the limit is taken as,

$$
\lim _{r \rightarrow 0} \frac{1}{\mu} \frac{d P}{d z} \frac{r^{2}}{4}+\frac{1}{\mu} C_{1} \ln (r)+C_{2}
$$

For the velocity to be finite at $r=0$, the first constant of integration must be zero $\left(C_{1}=0\right)$. The velocity equation is then reduced to:

$$
w=\frac{1}{\mu} \frac{d P}{d z} \frac{r^{2}}{4}+C_{2}
$$

The second boundary condition represents a continuation of the bulk velocity profile beyond the geometric constraints of the capillary tube. The differences of the flow boundary conditions are illustrated in Figure 11.

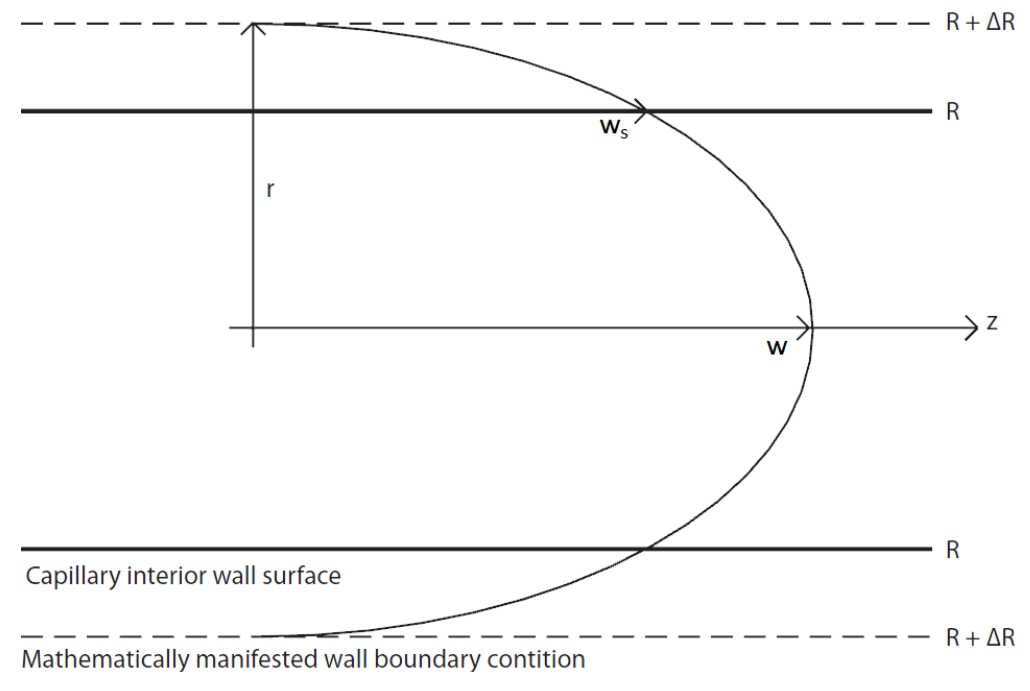

Figure 11: A graphical representation of the potential wall slip.

The introduced term $\Delta R$, represents a physical shift in the tube radius to accommodate the increased flow potential over that of a no slip condition. If this adjustable radius term $(\Delta R)$ is set to zero, the velocity equation reduces to that of a no slip case. Applying Boundary Condition (16) to Equation (18) and manipulating, the second 
constant of integration is obtained as:

$$
C_{2}=-\frac{1}{4 \mu} \frac{d P}{d z}(R+\Delta R)^{2}
$$

The velocity profile in the tube is then:

$$
w=\frac{1}{4 \mu} \frac{d P}{d z}\left(r^{2}-\Upsilon^{2}\right)
$$

where,

$$
\Upsilon=R+\Delta R
$$

\subsubsection{Averaged capillary flow}

Though the velocity of the flow in the capillary is well described with respect to the radial position, it does little to help in predicting the complete motion of the liquidvapour interface in the capillary. To better describe the motion of the interface, the liquid flow velocity is averaged with respect to the radial direction.

To do this, the velocity profile $(w)$ is integrated over the area of the tube so as to achieve an average liquid capillary velocity $(\bar{w})$. The average velocity in the tube is obtained by equating the volumetric flow rates in the tube. Starting with the volumetric flow rate for the velocity profile of Equation (20):

$$
\frac{d \forall}{d t}=\iint_{A} w d A
$$

Substituting Equation (20) into Equation (22) and integrating, the volumetric flow rate is then:

$$
\frac{d \forall}{d t}=\frac{-\pi}{8 \mu} \frac{d P}{d z} \Upsilon^{4}
$$

Next, the volumetric flow rate is obtained for the average capillary velocity through 
integration:

$$
\frac{d \forall}{d t}=\iint_{A} \bar{w} r d A
$$

As the averaged velocity in the capillary is constant with respect to the radial direction, the following volumetric flow rate is utilised to obtain the average velocity.

$$
\frac{d \forall}{d t}=\pi \bar{w} \Upsilon^{2}
$$

Therefore, the average flow velocity $(\bar{w})$ in the capillary of radius $R$, is obtained by equating Equations (23) and (25). Thus, the average velocity in tube is described by:

$$
\bar{w}=\frac{-1}{8 \mu} \frac{d P}{d z} \Upsilon^{2}
$$

If the meniscus were assumed to take a planar form normal to the z-axis, it can be inferred that the liquid-vapour interface of the capillary will move at the same rate as described by Equation (26). To identify the interface as a separate entity from the flow field, the interface velocity will take the following form:

$$
\bar{w}=\frac{d h}{d t}=\frac{-1}{8 \mu} \frac{d P}{d z} \Upsilon^{2}
$$

\subsubsection{Pressures within a capillary}

The forces acting on a fluid within a capillary tube can be correlated as a pressure balance. The principal pressures acting within a heated capillary tube may include, but not limited to: viscous pressure drop, liquid pressure, capillary pressure, hydrostatic pressure and vapour recoil. The resulting pressure balance can then be described as,

$$
\begin{aligned}
& \Sigma P=0 \\
& P_{l}+P_{c}+P_{h}+P_{v r}+P_{v i s}=0
\end{aligned}
$$




\section{Viscous pressure drop}

When the liquid is moving within the capillary tube, a viscous pressure drop will be present. The viscous pressure drop is not only present when the interface is moving but when the liquid within the capillary moves. For a meniscus that is steadily evaporating, for the interface to remain stationary, a replenishing supply of liquid is required, which incurs a viscous pressure drop.

By rearranging Equation (26), we can obtain the viscous pressure drop along the capillary tube as a function of the average fluid flow velocity. Integrating Equation $(26)$,

$$
\int_{0}^{z} \bar{w} d z=\frac{-1}{8 \mu} \Upsilon^{2} \int_{P}^{P+\Delta P} d P
$$

The viscous pressure drop for the liquid is found as:

$$
\Delta P=P_{v i s}=\frac{-8 \mu}{\Upsilon^{2}} \bar{w} z
$$

\section{Liquid pressure}

The liquid pressure stems from the momentum of the fluid moving in the capillary. This can be obtained through the application of Newton's second law. The mass of the fluid element within the capillary is:

$$
m=\rho A z
$$

and the mass flow rate is:

$$
\frac{d m}{d t}=\rho A \bar{w}
$$


Substituting Equation (31) and (32) into Newton's second law, applying the definition of pressure and simplifying, one obtains the liquid pressure as:

$$
P_{l}=-\rho \frac{d}{d t}(z \bar{w})
$$

\section{Capillary pressure}

The capillary pressure is taken to be a function of surface tension and curvature as:

$$
P_{c}=\sigma\left(\frac{1}{r_{1}}+\frac{1}{r_{2}}\right)
$$

As the capillary geometry is that of a cylinder, the two principal radii are equal $\left(r_{1}=r_{2}=R\right)$. Thus the capillary pressure is then:

$$
P_{c}=\frac{2 \sigma}{R}
$$

\section{Hydrostatic pressure}

The hydrostatic pressure comes simply as,

$$
P_{h}=-\rho g z
$$

where the negative sign is a result of the gravitational acceleration acting in the negative z-direction.

\section{Vapour recoil}

The vapour recoil is the result of the momentum exchange across the interface due to phase change. If the phase change process across the interface of the capillary is 
considered, the conservation of momentum can be applied across the interface:

$$
j=(m \mathbf{V})_{l}=(m \mathbf{V})_{v}
$$

The mass of the fluid state is represented as a function of the density and volume of the capillary as, $m=\rho A h$. The velocity of the fluid state $(\mathbf{V})$ is one that is relative to the position of the interface. To address the motion of the fluid with respect to the relative motion of the liquid-vapour interface the following relation can be implemented as:

$$
V_{l}=w_{l}-\frac{d h}{d t}
$$

and,

$$
V_{v}=w_{v}-\frac{d h}{d t}
$$

Combining the relative velocity relation and the momentum balance across the interface the kinematic boundary condition for the mass flux is obtained as:

$$
j=\rho_{l}\left(w_{l}-\frac{d h}{d t}\right)=\rho_{v}\left(w_{v}-\frac{d h}{d t}\right)
$$

The mass flux relations must then be multiplied by their respective velocities to obtain the momentum flux, commonly referred to as vapour recoil. Thus, the vapour recoil is described as:

$$
P_{v r}=j\left(\mathbf{V}_{l}-\mathbf{V}_{v}\right)=\rho_{l} w_{l}\left(w_{l}-\frac{d h}{d t}\right)-\rho_{v} w_{v}\left(w_{v}-\frac{d h}{d t}\right)
$$

Finally, the vapour recoil can be further reduced by the substitution of Equation (40), and simplified to,

$$
P_{v r}=-j^{2}\left(\frac{\rho-\rho_{v}}{\rho \rho_{v}}\right)
$$


The vapour recoil acting upon the liquid-vapour interface as described by Equation (42) is a macroscopic representation of the molecular effects acting upon the interface. Thus, the vapour-recoil effect is described effectively as a macroscopic pressure. It is also important to note that the vapour recoil always acts against the liquid interface for either evaporation or condensation.

\subsection{The interface}

The motion of the interface is governed by Equation (28), and (26). If the respective pressures are replaced with those derived previously, the following is obtained:

$$
\rho \frac{d}{d t}(h \bar{w})=\frac{2 \sigma}{R}-\rho g h-\bar{w} \frac{8 h \mu}{\Upsilon^{2}}-\frac{\rho-\rho_{v}}{\rho \rho_{v}} j^{2}
$$

Though this form of the governing equation is accurate, it does little to explain the dynamics of the interface with respect to the evaporation. To this end, the average velocity can be substituted with the kinematic boundary condition of Equation (40),

$$
\rho \frac{d}{d t}\left(h \frac{d h}{d t}+h \frac{j}{\rho}\right)=\frac{2 \sigma}{R}-\rho g h-\frac{\rho-\rho_{v}}{\rho \rho_{v}} j^{2}-\frac{8 \mu}{\Upsilon^{2}}\left(h \frac{d h}{d t}+h \frac{j}{\rho}\right)
$$

The motion of a meniscus subject to phase change is governed by the following second order, ordinary differential equation:

$$
\frac{d^{2} h}{d t^{2}}=\frac{2 \sigma}{\rho h R}-g-\left(\frac{\rho-\rho_{v}}{\rho \rho_{v}}\right) \frac{j^{2}}{\rho h}-\frac{j}{\rho h} \frac{d h}{d t}-\frac{1}{h}\left(\frac{d h}{d t}\right)^{2}-\frac{8 \mu}{\Upsilon^{2} \rho}\left(\frac{j}{\rho}+\frac{d h}{d t}\right)-\frac{d j}{d t} \frac{1}{\rho}
$$




\subsubsection{Phase change model}

The conditions which dictate the phase change have been generalised and require further development. As environmental conditions may lead to changes in the evaporation or condensation a phase change model must be used.

Though the interface of the meniscus is expected to be of a curved nature, the model derivation assumes the interface to be flat. As such, the Clapeyron phase change model is used herein, as it is consistent with the study by Ramon and Oron [47] with the assumption that $T_{l} \approx T_{l v}$.

$$
j=a\left(T_{l v}-T_{v}\right)=a \Delta T
$$

where,

$$
a=\left(\frac{C^{2} M}{2 \pi R T_{l v}}\right)^{1 / 2}\left(\frac{P_{v} M h_{f g}}{R T_{v} T_{l v}}\right)
$$

Implementation of the Clapeyron phase change model allows for the defining of the evaporation mass flux as a function of fluid properties and superheat.

\subsection{Steady state meniscus}

The principle aspects governing meniscus height and motion are captured in Equation (45). To begin the analysis of the model, we first consider the steady state conditions.

Under steady state conditions, the meniscus will reside at some equilibrium height consistent with Equation (45), where the height derivatives and evaporative transients are assigned a value of zero, giving:

$$
H_{e q}=\frac{\frac{2 \sigma}{R}-\left(\frac{\rho-\rho_{v}}{\rho \rho_{v}}\right) j^{2}}{\rho g+\frac{8 \mu j}{\Upsilon^{2} \rho}}
$$


The resulting equation for the meniscus equilibrium height is consistent with Ramon and Oron [47], with the exception that the ratio of liquid and vapour densities is retained. Thus, the equilibrium height is a function of the fluid properties and the rate of evaporation.

While much focus has been placed on the meniscus dynamics and how the system will respond to different perturbations, their remains the superheat associated with the phase change model. A rearrangement of Equation (48) allows for the expression of the superheat as a function of the steady state meniscus height:

$$
a^{2}\left(\frac{\rho-\rho_{v}}{\rho \rho_{v}}\right) \Delta T^{2}+\left(\frac{8 a \mu h}{\rho \Upsilon^{2}}\right) \Delta T+\rho g h-\frac{2 \sigma}{R}=0
$$

This relation provides a critical link between theory and any potential experiment. Using Equation (49), it is possible to correlate a steady state meniscus height with superheat.

\subsection{Numerical solution}

The non-linear nature of Equation (45) is beyond analytical methods and requires a numerical integration scheme. Equation (45) is reduced to a set of first order differential equations and solved using an implicit Runge-Kutta method. The governing equation is reduced using the following transformations:

$$
\begin{gathered}
h=\psi_{0} \\
\frac{d h}{d t}=\psi_{1}
\end{gathered}
$$


Thus, the two first order equations are:

$$
\begin{gathered}
\frac{d \psi_{0}}{d t}=\psi_{1} \\
\frac{d \psi_{1}}{d t}=\frac{2 \sigma}{\rho \psi_{0} R}-g-\left(\frac{\rho-\rho_{v}}{\rho \rho_{v}}\right) \frac{j^{2}}{\rho \psi_{0}}-\frac{j \psi_{1}}{\rho \psi_{0}}-\frac{\left(\psi_{1}\right)^{2}}{\psi_{0}}-\frac{8 \mu}{\Upsilon^{2}}\left(\frac{j}{\rho^{2}}-\frac{\psi_{1}}{\rho}\right)=0
\end{gathered}
$$

Verification of the model, Equation (53), was performed through a comparison with the model developed by Ramon and Oron [47]. To complete the verification all of the fluid properties and perturbations were required. Though Ramon and Oron [47] published most of the fluid properties, the vapour density and height perturbation values used were omitted. To address this shortcoming, the equilibrium height equation was used in concert with a graphical plot from Ramon and Oron [47] to obtain the vapour density. The results of this investigation produced vapour density values of $\rho_{v_{w}}=0.902$ and $\rho_{v_{D E}}=2.443$. Thus, the fluid properties used for the purposes of verification are provided in Table 1.

Table 1: Fluid properties consistent with Ramon and Oron [47]

\begin{tabular}{l|llllll}
\hline Fluid & $\rho$ & $\rho_{v}$ & $\mu$ & $\sigma$ & $h_{f g}$ & $M$ \\
& $\left(\mathrm{~kg} / \mathrm{m}^{3}\right)$ & $\left(\mathrm{kg} / \mathrm{m}^{3}\right)$ & $(\mathrm{kg} / \mathrm{m} \cdot \mathrm{s})$ & $(\mathrm{N} / \mathrm{m})$ & $(\mathrm{kJ} / \mathrm{kg})$ & $(\mathrm{kg} / \mathrm{kmol})$ \\
\hline Water & 997 & 0.902 & $9.11 \times 10^{-4}$ & 0.072 & 2360 & 18.02 \\
Diethyl Ether & 708 & 2.443 & $2.23 \times 10^{-4}$ & 0.016 & 377 & 74.0 \\
\hline
\end{tabular}

While the vapour density was obtained using provided data, the height perturbation used by Ramon and Oron [47] was not published. The perturbation applied to the height was visually approximated by comparing the numerical results and the published data and figures of Ramon and Oron [47]. An applied height perturbation of $1 \times 10^{-3} \mathrm{~m}$ was found to best fit the published data. 


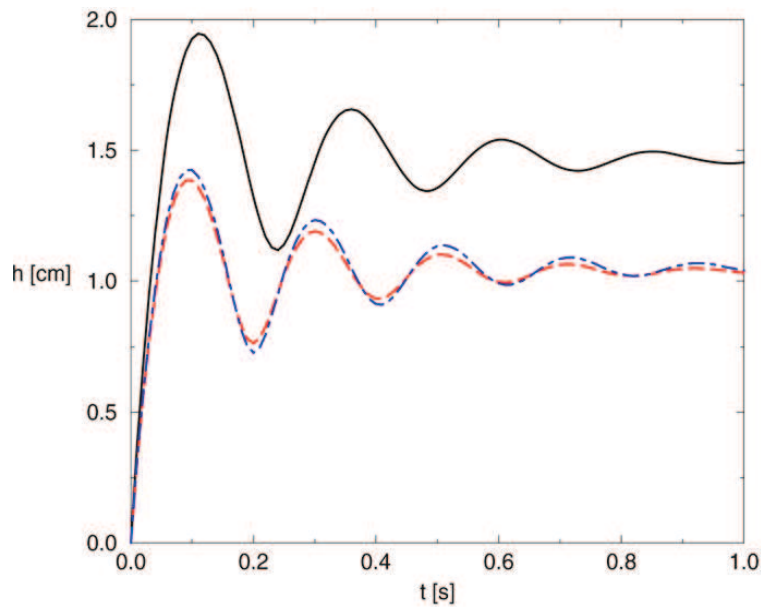

(a) Ramon and Oron [47].

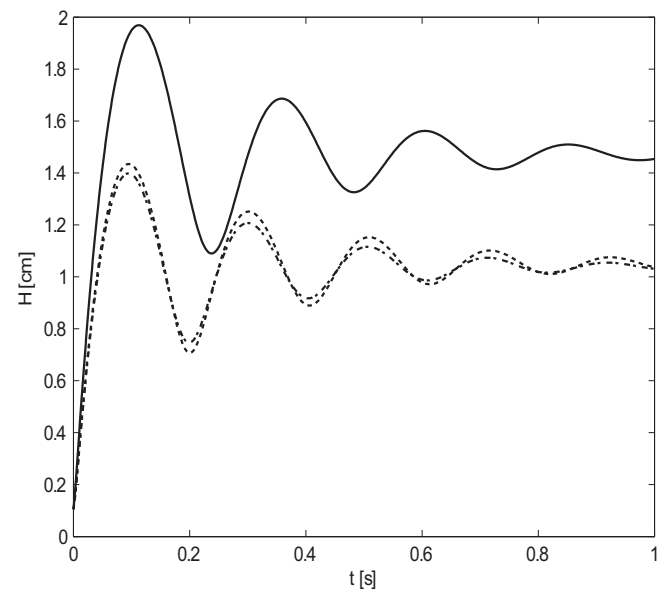

(b) Current model.

Figure 12: Model verification for water.

To confirm the consistency of the MATLAB solution with the work of Ramon and Oron [47], the motion of the capillary interface was plotted for all cases presented. Applying the assumptions of Ramon and Oron [47], the MATLAB implementation comparable results as presented in Figure 12.

The solid lines indicate the rise of a non-evaporating meniscus, while the dashed and dotted lines represent the dynamics of an evaporating and condensing meniscus respectively. Though the results are consistent, the vapour density fluid properties used by Ramon and Oron [47] are not consistent with published values [115]. Thus, in the following flow analyses the fluid properties are consistent with those published in the CRC Handbook [115].

\subsection{Summary}

A mathematical model describing the dynamics of a meniscus was developed and was able to account for how phase change affects meniscus position and dynamics. 
The model was that of a non-linear differential equation, from which it was possible to determine the equilibrium height for a set of conditions. Investigating beyond the simple meniscus dynamics, it was discovered in this work that the model could be used to determine the superheat from a measurement of meniscus height. This fundamental finding provides a means by which to perform non-invasive experimental measurements and correlate them to a superheat value consistent with current theoretical models. 


\section{Chapter 4}

\section{Meniscus dynamics - experiments}

Having developed a theoretical basis of describing the motion of a meniscus constrained within a capillary tube, the next logical step is to perform a validation

study. To this end, a series of experiments were performed for a cylindrical capillary tube subject to a heating load. The experiments were designed to study the meniscus dynamics for the conditions of thermal equilibrium and evaporation. The theoretical formulation and experimental design were carefully developed so as to maintain the greatest degree of consistency between the theory and experiments.

\subsection{Experimental setup}

The single species assumption required an experimental setup that isolated the experiments from the ambient. To achieve this, an experimental setup was devised where a bell jar vacuum chamber was used as shown in Figure 13. 


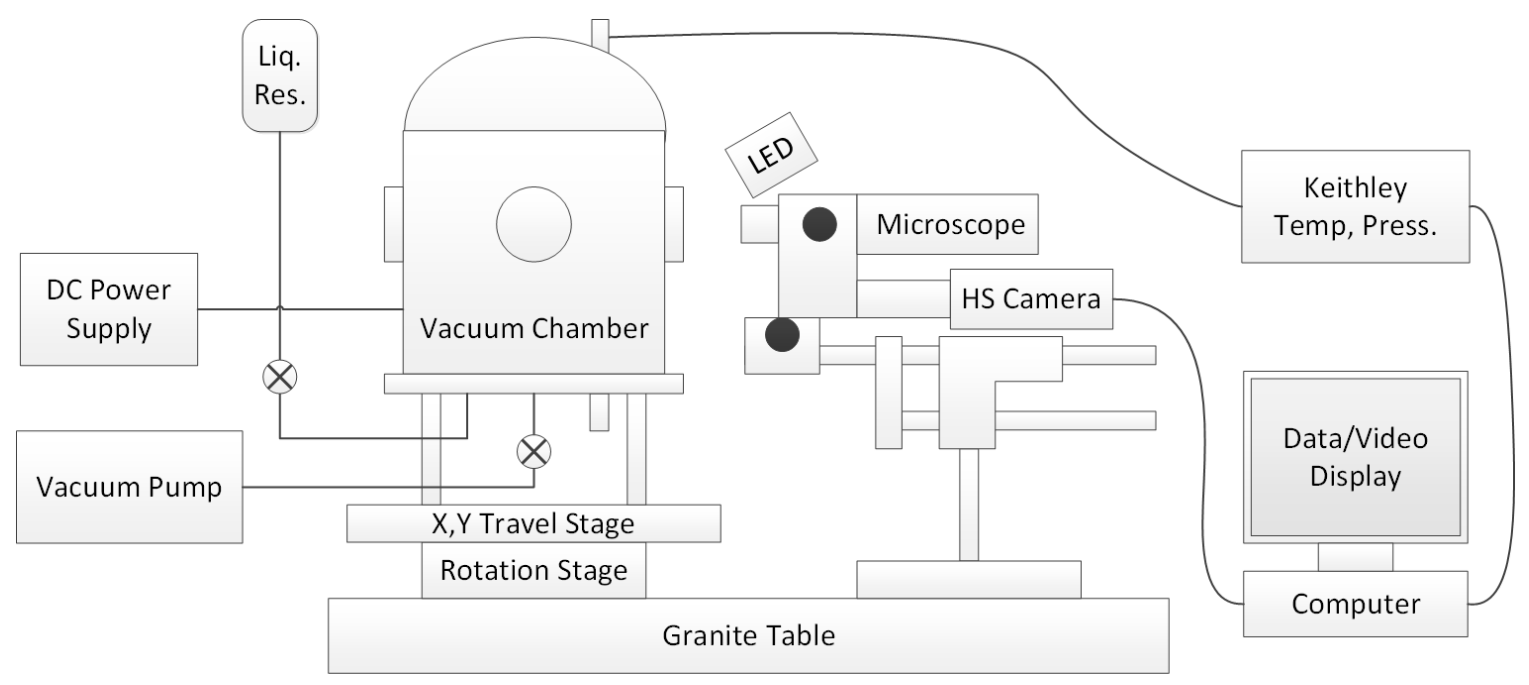

Figure 13: Experimental setup.

A stainless steel bell jar vacuum chamber and a roughing pump (Alcatel 2005 SD) were used to maintain a saturated single species environment. The vacuum chamber was instrumented with a pressure transducer (Omega PX429-015A5V) with an accuracy of $\pm 0.1 \%$ and a thermocouple (Omega 5SRTC-GC-T-30-36) with an accuracy of $\pm 0.5 \mathrm{~K}$. The thermocouple and pressure transducer were connected to a Keithley 2700 DAQ 22 bit analogue-to-digital converter with an accuracy of $\pm 0.87 \%$. The vacuum chamber assembly was installed atop a levelled granite table. The granite table was installed upon a sand bed that was in turn placed on airbags and rubber vibration dampers. The airbags and rubber vibration dampers were necessary to mitigate ambient vibrations from reaching the experiments.

The vacuum chamber had three observation view ports and liquid, mechanical, electrical and thermocouple feed-throughs. Visual observation of the experiments inside the vacuum chamber was performed using an Olympus SZH-10 optical stereomicroscope. The optical setup of the stereo-microscope was dependent upon the rise height of the liquid and by extension the diameter of the capillary tubes. The lenses used for the experiments are listed in Table 2. 
Table 2: Capillary tube and lens pairings.

\begin{tabular}{l|l} 
Capillary Tube Diameter & Optical Lens \\
\hline $0.5 \mathrm{~mm}$ & Olympus DFPL $0.5 \times 4$ \\
$1.0 \mathrm{~mm}$ & Olympus DF Planapo $1 \times$ \\
$2.0 \mathrm{~mm}$ & Olympus DF Planapo $1 \times$
\end{tabular}

The stereo-microscope was equipped with a MotionScope M1 high speed camera capable of 1000 frames per second (fps). As the frame rate increases the exposure time is reduced, thereby leading to dark images. Using the meniscus dynamics (imbibition) model developed previously in Chapter 3, a frame rate of $250 \mathrm{fps}$ was selected. To aid in the observation of the capillary tubes, an Edmund NT66-847 high intensity LED was used for illumination. The high speed camera was fitted with a Fujinon CF25HA-1 1:1.4/25mm TV lens and installed so as to have the same view as seen through the microscope eye-piece.

To study the meniscus dynamics inside the vacuum chamber, a motion platform was installed to allow for the dipping of capillary tubes into the liquid reservoir as illustrated in Figure 14.

To expedite the testing, five capillary tubes of the same diameter were arranged to form a linear array. Three different capillary tube arrays were used in the experiments with nominal internal diameters of: $0.5 \mathrm{~mm}, 1.0 \mathrm{~mm}$ and $2.0 \mathrm{~mm}$. Each capillary tube array was assembled with the tube ends flush and epoxied together using a chemically resistant epoxy (Devcon HP 250). The capillary tubes were cleaned using an ultrasonic acetone bath and dried on a hot plate for a 30 minute period.

The motion platform inside the vacuum chamber was rigged with a Katpon heater (Omega KHLV-102/10) with a resistance of $37.8 \Omega$. The face of the heater was 


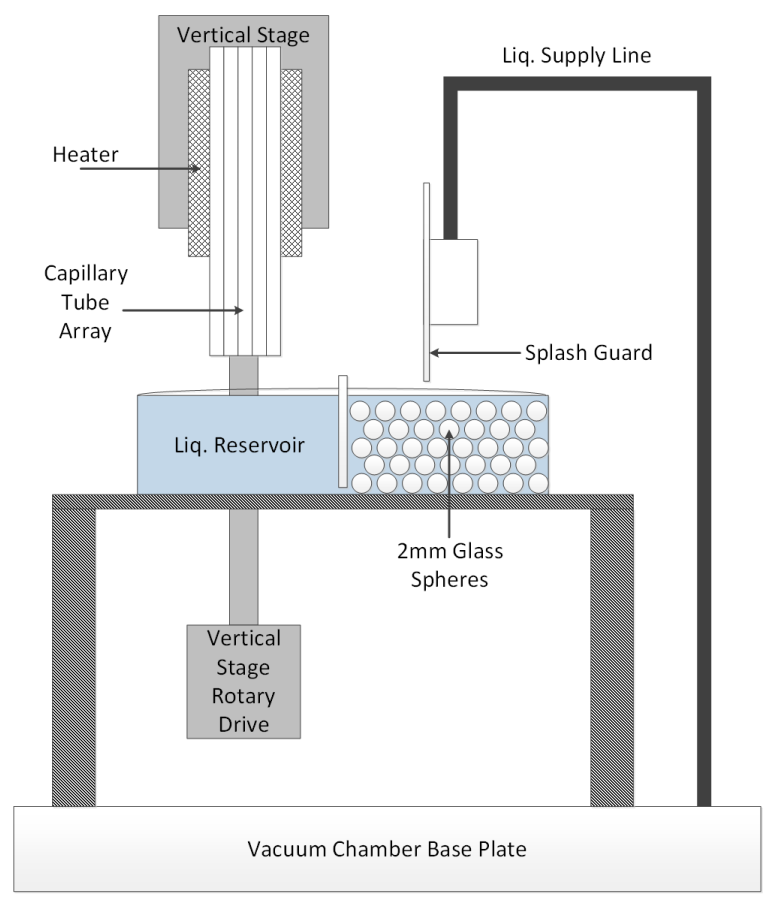

Figure 14: Internal experimental setup.

placed on the lowering arm where it would be in direct contact with the back side of the capillary tube array. The motion platform was actuated manually through the mechanical rotary feed-through on the base of the vacuum chamber.

The liquid reservoir was positioned directly below the capillary tube array. The reservoir was divided into two sections with one half filled with $2 \mathrm{~mm}$ glass spheres. The glass spheres served to dampen the fluid motion in the reservoir during filling. The divider was installed to prohibit the propagation of surface waves from the filling side to the testing side of the reservoir.

All components inside the vacuum chamber were cleaned using acetone and dried using a heat gun and blown down with high purity dry Nitrogen. The experiments were performed using three different working fluids (n-pentane, iso-octane and acetone) as supplied without modification. The fluids were selected based on their thermophysical properties and volatility at ambient temperatures. The experimental test plan is given in Table 3 . 
Table 3: Experimental parameters.

\begin{tabular}{l|l} 
Fluids & n-pentane, iso-octane and acetone \\
\hline Tube Diameter $(\mathrm{mm})$ & $0.5,1.0$ and 2.0 \\
\hline Heater Power $(\mathrm{W})$ & $0,0.7,2.7,6.0,10.6$
\end{tabular}

\subsection{Experimental procedure}

The whole vacuum chamber setup was cleaned and assembled for each and every test performed. In accordance with the experimental test plan, a capillary tube array was installed on the lowering arm inside the chamber with the tubes long axis aligned with gravity using a digital level with an accuracy of \pm 0.1 deg. A thermocouple was then placed on the surface of the capillary tubes, opposite the heater. The reservoir was then installed with the divider and glass beads and the mechanical feed-through hooked up.

The vacuum chamber was sealed and a vacuum of at least 50 mTorr was drawn. The chamber was then sealed off using a line valve from the outside environment. The external working fluid supply was filled and the chamber charged and reservoir filled with working fluid. The system pressure and temperature were monitored until steady state was achieved. Any losses from the liquid reservoir were replenished prior to the experiment.

The capillary tube array was then observed using the stereo-microscope for any signs of splash from the reservoir. If the tubes were not dry, they were heated using the Kapton heater and the fluid evaporated off. Once dry, the capillary tube array was then permitted to cool to the ambient chamber temperature and reach a steady state condition. The tubes were then mechanically lowered near the free surface of 
the reservoir.

The Keithley 2700 DAQ and MotionScope M1 high speed camera were initialised and the capillary tube array lowered into the reservoir. The liquid was observed to rise in the capillary tubes and permitted for a period of time to achieve steady state. The data was saved and prepared for post analysis. The capillary tube array was then raised from the reservoir and heated using the Kapton heater to evaporate any remaining working fluid. The tubes were permitted a period of cooling until achieving steady state.

Each subsequent test for the capillary tube array was subject to a prescribed heater load as outlined in Table 3. Upon the start of the heated tests, the Kapton heater was turned on and the temperature monitored for steady state. Once the capillary tube array achieved steady state, the DAQ and high speed camera were initialised and the tubes lowered into the liquid reservoir. The aforementioned procedure was performed for all five heater power settings in succession.

Once a capillary tube array experimental set had been completed, the tubes were dried in the chamber and the heater powered off. The system was permitted a cooling period prior to opening to the outside environment so as to reduce the risk of fire. The procedure was repeated for each capillary tube array set, working fluid and heater power setting with cleaning between each test.

\subsection{Post processing techniques}

The data collected using the high speed camera was stored in a raw video format. Using the proprietary MotionScope M1 software, the desired frames from each experimental test were extracted and converted into bitmap format. The image sequences were then re-sized and digitally enhanced using ImageJ. As the capillary tubes were vertically aligned in the frame, each tube's axis was confined to a single column of 
pixels. The raw image data was then run through a custom MATLAB code to extract the location of the bottom of the meniscus. The data was then filtered using a custom speckle filter.

The spatial resolution of each pixel was correlated using the capillary tube outer diameter. The meniscus position information was found to have a tolerance of \pm 1 pixel which corresponds to positional errors of $\pm 0.07 \mathrm{~mm}$ for $0.5 \mathrm{~mm}$ capillaries and $\pm 0.05 \mathrm{~mm}$ for $1.0 \mathrm{~mm}$ and $2.0 \mathrm{~mm}$ capillaries. The meniscus position was then shifted up by $R / 3$ to conform with the average height used in the model developed in Chapter 3.

\subsection{Experimental results}

As the capillary array made contact with the liquid reservoir, the working fluid wet the capillary tube wall and proceeded to climb up the capillary tube. The motion of the interface was captured with the high speed camera and the height values were extracted using the custom MATLAB code as outlined previously.

\subsubsection{Differences attributed to heating}

Given the information provided in Chapter 3, the meniscus rise profiles varied depending upon the size of the capillary, the working fluid and the heat load applied. The typical rise profiles for heated iso-octane are shown in Figures 15 - 17, for capillary tubes with diameters $0.5,1.0$ and $2.0 \mathrm{~mm}$ respectively. 


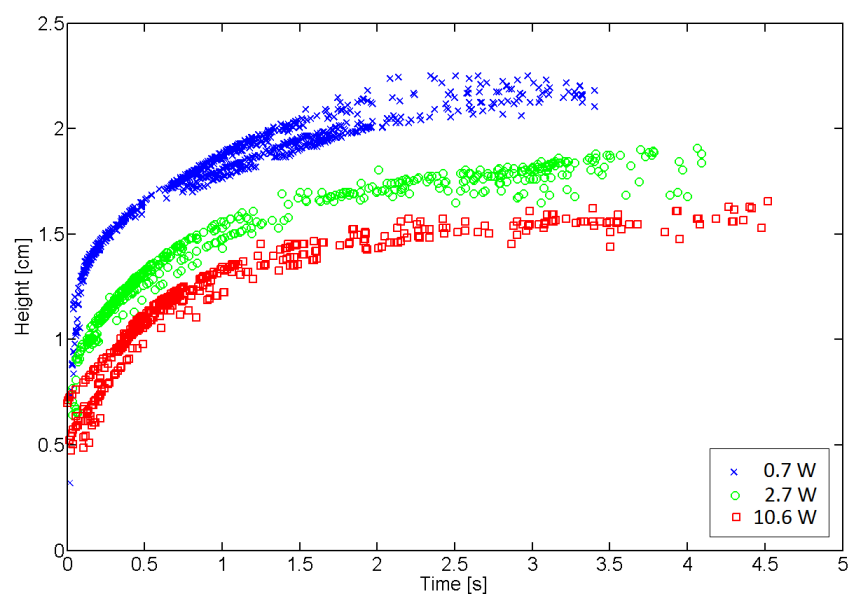

Figure 15: $0.5 \mathrm{~mm}$ capillary tube with heated iso-octane.

As the $0.5 \mathrm{~mm}$ capillary is brought into contact with the interface, the liquid wets the capillary walls and begins to rise rapidly. The rise of the fluid in the capillary is consistent between the three heating loads illustrated. As the liquid rises up the capillary it begins to decelerate and tend toward an equilibrium height, akin to an overdamped system. The point at which the meniscus begins to decelerate and the equilibrium height are clearly seen to be a function of the applied heating load as shown in Figure 15. The equilibrium height is shown to decrease with an increased heater load and is consistent with the theoretical analysis presented in Chapter 3.

When the capillary tube diameter is doubled to that of $1.0 \mathrm{~mm}$, the meniscus dynamics are notably changed. As can be seen in Figure 16, the meniscus begins its rise rapidly as before, but then proceeds to overshoot what is to be the equilibrium height. The meniscus undergoes a single oscillation of decreasing amplitude before it rests at the equilibrium height. 


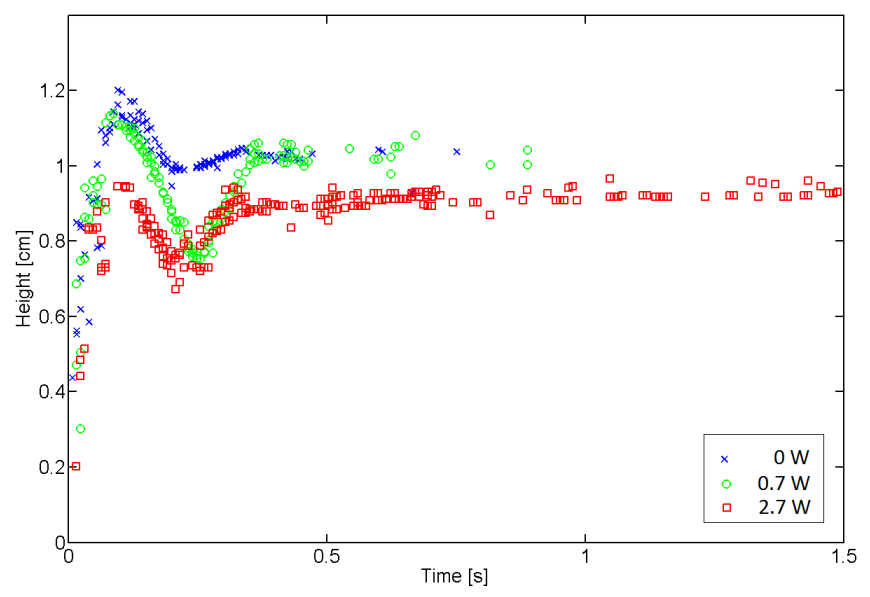

Figure 16: $1.0 \mathrm{~mm}$ capillary tube with iso-octane.

The differences in the meniscus dynamics attributed to the heater load are small and appear most prominent in the early portions of the motion. As is shown in Figure 16, the unheated case has the largest amplitude of overshoot. The magnitude of the overshoot is reduced for the $0.7 \mathrm{~W}$ case and is further reduced for the $2.7 \mathrm{~W}$ case. This reduction in oscillation amplitude is observed throughout the meniscus motion trajectories as each heater condition is observed to reach a different equilibrium height.

Doubling the capillary tube diameter again to $2.0 \mathrm{~mm}$, the nature of the meniscus dynamics differs slightly from that of the $1.0 \mathrm{~mm}$ tube. The meniscus rises and overshoots as before, with the oscillation persisting for four cycles before settling at the equilibrium height. 


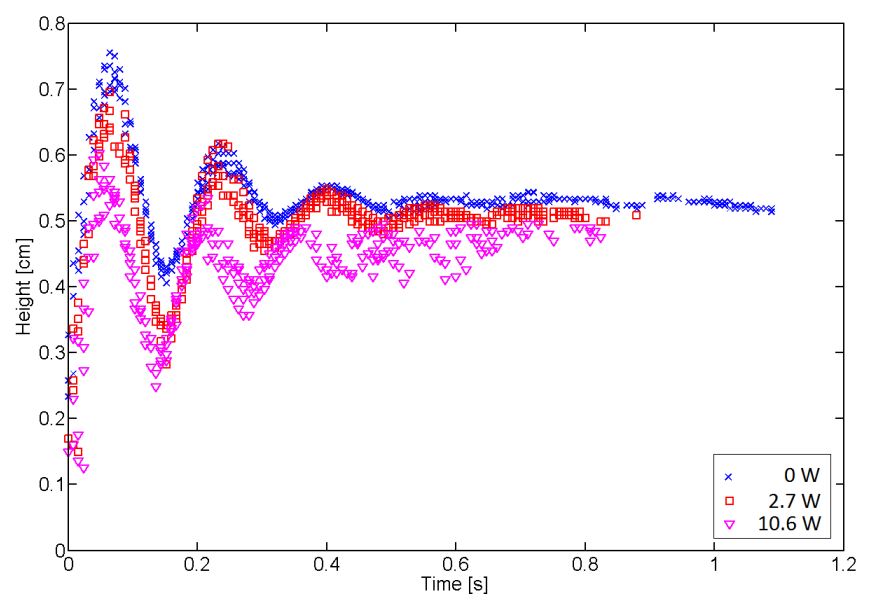

Figure 17: $2.0 \mathrm{~mm}$ capillary tube with iso-octane.

When heat is applied to the $2.0 \mathrm{~mm}$ capillary tube, the meniscus is observed to demonstrate a similar behaviour to that of the $1.0 \mathrm{~mm}$ tube as seen in Figure 17. The unheated case has the largest overshoot and the $10.6 \mathrm{~W}$ case has the smallest overshoot. With the increase in heater power, a non-linear shift in the oscillating phase becomes apparent, as seen in the $10.6 \mathrm{~W}$ heating case. The differences in oscillation amplitudes and phase continue through the meniscus dynamics to the final equilibrium height.

\subsubsection{Differences attributed to the fluid}

The experiments utilised acetone, n-pentane and iso-octane as the working fluids. The different fluids provided some differences in the fluid properties, with acetone introducing polarity. Using the $2.0 \mathrm{~mm}$ capillary tubes as a basis for comparison of the three fluids, Figures 18 and 19 illustrate the differences for both unheated and heated cases respectively. 


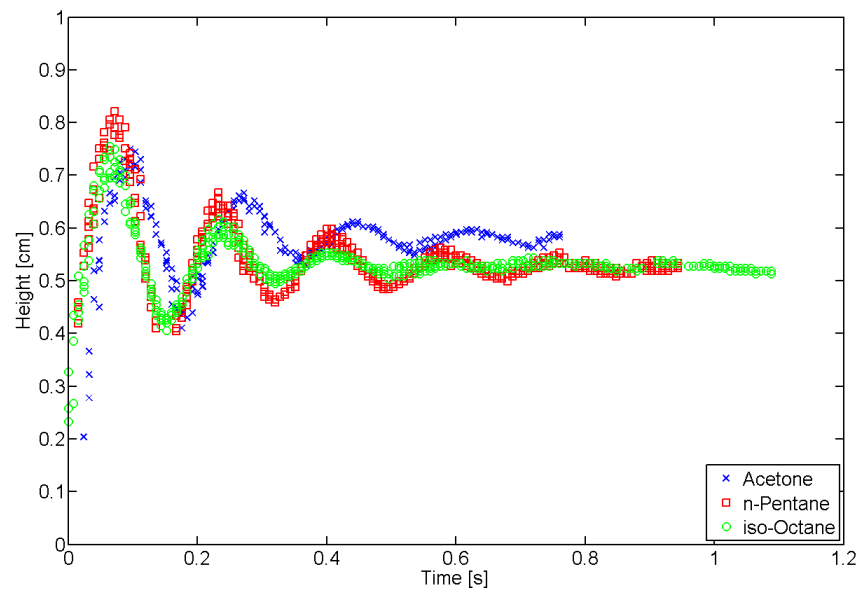

Figure 18: Unheated $2.0 \mathrm{~mm}$ capillary tube meniscus heights.

When the capillary tube is unheated, Figure 18, the meniscus dynamics for the n-pentane and iso-octane are comparable in oscillation amplitude, phase and final equilibrium height. The amplitude of the iso-octane is noted to be consistently less than that of the n-pentane. The acetone dynamics exhibit a similar oscillatory nature but differ in the amplitude, phase and equilibrium height as compared to the npentane and iso-octane. The difference in equilibrium height is expected due to the differences in surface tension.

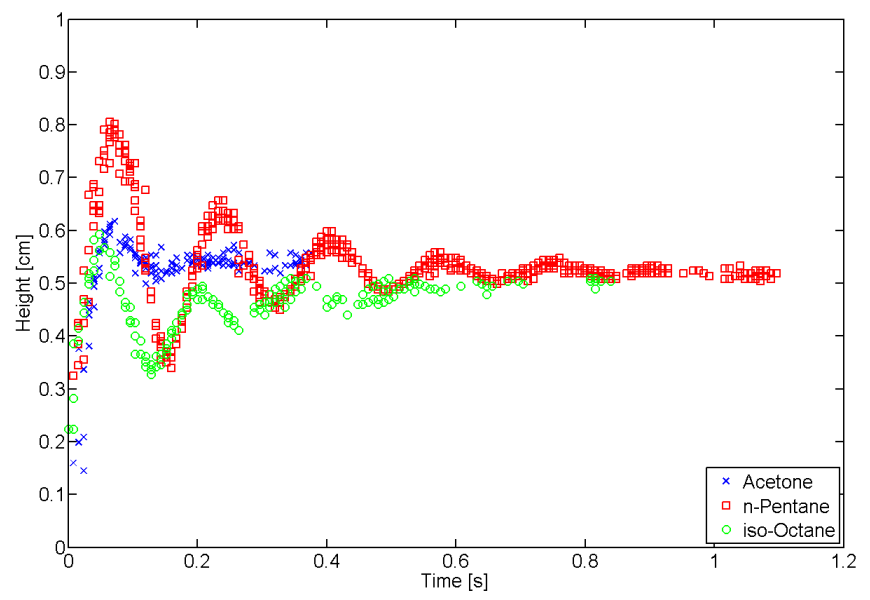

Figure 19: Heated (6 W) $2.0 \mathrm{~mm}$ capillary tube meniscus heights. 
When the capillary tubes are heated with an applied load of $6 \mathrm{~W}$, the meniscus dynamics become unique to each fluid. As can be observed in Figure 19, when the heat is applied the n-pentane maintains a similar oscillation profile as it had for the unheated case. The iso-octane however experiences a significant change in the oscillation amplitude and phase, with little change to the final equilibrium height.

The change in the heated meniscus oscillation is significantly more pronounced for the acetone case. The acetone appears to rise as before and achieves an overshoot less than that achieved in the unheated case, approximately $20 \%$ less in this example. The overshoot is then observed to quickly return to the final equilibrium height with very little oscillation. From this comparison it is clear that changes in fluid properties can lead to significant differences in the way the meniscus behaves in unheated and heated conditions.

\subsubsection{Factor of merit}

The n-pentane and iso-octane are both alkanes and share the same principal structure $\left(\mathrm{C}_{5} \mathrm{H}_{10}\right)$, with iso-octane having three methyl groups $\left(\mathrm{CH}_{3}\right)$. As such, it is not surprising that they had similar behavioural characteristics as previously presented. However, differences in their behaviour for the heated case are of some interest and extend to the significant differences observed for acetone.

To draw some basis of comparison, a factor of merit $(F M)$ is proposed. Given the contributing factors in the analysis, the principal aspects are thought to be surface tension, latent heat and kinematic viscosity. Larger values of surface tension and latent heat serve to increase the height, while the viscous effects are resisting the motion and act against the rise of fluid. As such the factor of merit is formulated as:

$$
F M=\frac{\sigma h_{f g}}{\nu}
$$


To better compare the factor of merit between the three fluids, it can be normalised. The normalised factor of merit, $(\overline{F M})$, is obtained using the acetone as the principal divisor for the three fluids tested. The factor of merit and it's normalised form are presented in Table 4.

Table 4: Factor of merit for each fluid tested.

\begin{tabular}{l|l|l} 
Fluid & $F M$ & $\overline{F M}$ \\
\hline n-pentane & $1.64 \times 10^{7}$ & 0.51 \\
iso-octane & $6.81 \times 10^{6}$ & 0.21 \\
acetone & $3.25 \times 10^{7}$ & 1
\end{tabular}

As it can be seen, the three fluids are sufficiently different with respect to the normalised factor of merit. It can also be noted that n-pentane and iso-octane are comparative at 0.51 and 0.21 respectively, while acetone is 1 . This finding is generally consistent with the experimental results presented earlier in Figure 19.

\subsection{Model validation}

With the development of the imbibition model in Chapter 3 and the collection of experimental test data, a validation analysis is possible. The following outlines a comparison of the experimental data to the imbibition model with a few considerations. Firstly, the slip condition preserved in the model may be addressed based on the scales of the experiment.

The potential for a wall slip condition has been found to occur in nano and micro scale flows [65-67]. The experimental conditions which lead to such a condition differ from those observed in this experimental work. For the experiments performed, the 
slip condition is taken to be zero thereby enforcing a no-slip condition at the wall. This decision is based on the observed peak experimental flow rates where the Reynolds number was less than fifty $(R e \leq 50)$. Furthermore, as the capillaries were of sufficient size and did not have any surface treatments, a no-slip condition is assumed $(\Delta R=0)$ as is consistent with the findings of Pit et al. [91].

The experiments used a constant heater setting and a preheated capillary tube array to facilitate evaporation. Though the heater setting and steady state temperature were enforced, both occur at the outset of the experiment. As the liquid rose within the capillary tube, the heat was transfered from the glass capillary tube to the liquid and caused evaporation. Depending on the rate of the liquid rise and the heat transfer rate, two potential options become apparent: constant and/or transient evaporation. The constant evaporation rate is desirable as it is easily implemented in the model; however the transients introduce a greater deal of complexity.

As the liquid rises in the capillary tube for the first motion, the meniscus will be subject to a near constant wall temperature. However, once the liquid occupies the capillary, a convective heat transfer condition will occur. The capillary tube wall temperature will be reduced as the meniscus falls. Thus, the evaporation rate will be reduced on the meniscus descent. This transient transfer of heat from the capillary to the liquid will inevitably lead to a transient evaporative condition. This transient heat transfer interaction is beyond the scope of this work, therefore the evaporation transients are assumed to be of the form:

$$
f(t)=1-\exp \left(-\frac{t-t_{0}}{\tau}\right)
$$

and is implemented in the Clapeyron evaporation model as:

$$
j=f(t) a \Delta T
$$




\subsubsection{Superheat determination}

While the Clapeyron evaporation model is readily available, the superheat value is of a form that cannot be readily determined through traditional experimental methods. To formulate a reasonable comparison between the experimental results and theory we must apply an appropriate superheat value. As the imbibition model utilises the same superheat definition, it can be used to determine the superheat based on a simple measurement of the meniscus height with respect to the reservoir. While this can be done throughout the experiment, the height at which the meniscus settles can be used as a baseline value as discussed by Polansky and Kaya [116].

If we rearrange the governing equation from Chapter 3 and include a viscous pressure drop for the vapour phase, the superheat to meniscus height relation is obtained as:

$$
\left(\frac{\rho-\rho_{v}}{\rho \rho_{v}}\right) a^{2} \Delta T^{2}+\left(\frac{\mu h}{\rho v}+\frac{\mu_{v}(L-h)}{\rho_{v} v_{v}}\right) a \Delta T+\left(\rho g h-\frac{2 \sigma}{R}\right)=0
$$

Using Equation (57) and the experimental data, the superheat can be appropriately determined. A comparison of the rise in a $0.5 \mathrm{~mm}$ capillary for $\mathrm{n}$-pentane for the unheated (blue) and heated (red) cases are shown in Figure 20. As it can be seen, the imbibition model is able to accurately follow the rise trajectory for the unheated case. It is noted that the unheated and heated cases tend to the same final equilibrium height and hence they have the same baseline superheat.

As the final equilibrium height was used to determine the baseline superheat, the model trajectory is the same for both the heated and unheated cases. Furthermore, when the transient evaporation is applied (dashed line), the motion overshoots the constant evaporative case (solid line). Thus, it is noted that the model does not always correlate well with the dynamic motion of the meniscus as it rises. 


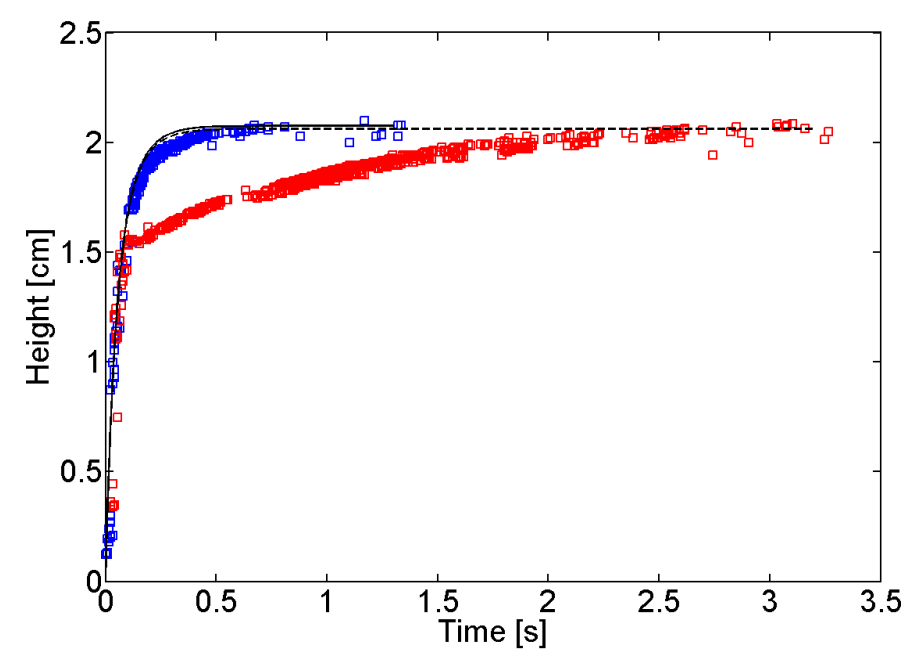

Figure 20: Unheated (blue) and heated at $6 \mathrm{~W}$ (red) capillary rise profiles for $\mathrm{n}$-pentane in a $0.5 \mathrm{~mm}$ capillary tube.

As the heated cases are the focus of this work, the following outlines a series of comparisons for the three fluids, three capillary tube arrays and a range of heating conditions. Continuing with the n-pentane comparison, Figures 21 and 22 are for the $1.0 \mathrm{~mm}$ and $2.0 \mathrm{~mm}$ capillaries respectively. In the case of the $1.0 \mathrm{~mm}$ tubes and a heater power of $6.0 \mathrm{~W}$, the motion is able to capture both the phase and amplitude of the oscillation.

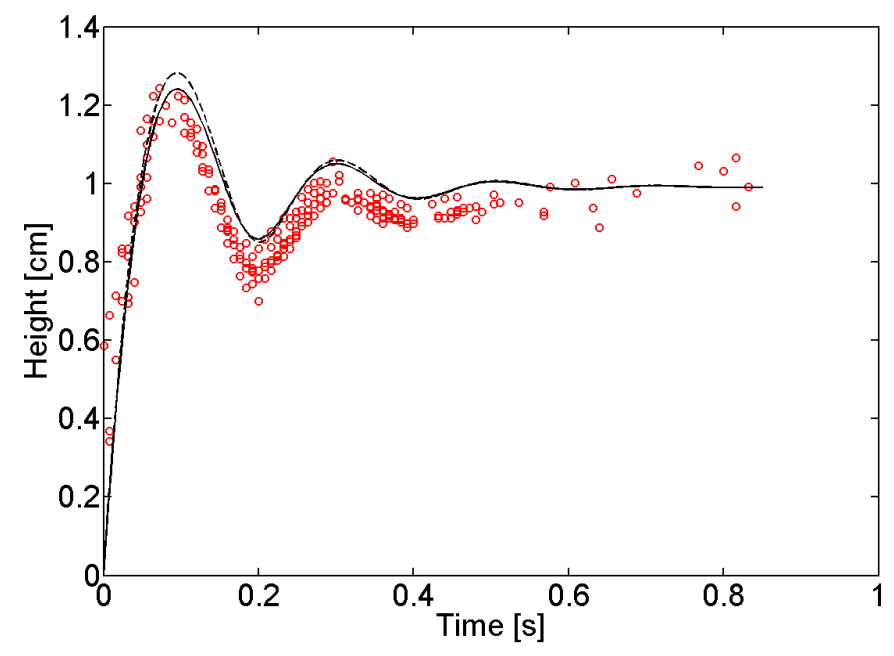

Figure 21: Heated $1.0 \mathrm{~mm}$ capillary rise profiles for n-pentane at $6.0 \mathrm{~W}$. 
For the $2.0 \mathrm{~mm}$ and $6.0 \mathrm{~W}$ heater cases, the alignment of the oscillation phase degrades with time due to non-linearities in the flow. This non-linear change in the oscillation is speculated to be a result of the one dimensional flow assumption imposed on the model. With the increase in the capillary size and the potential for flow reversal, the oscillation may lead to the development of a more complex flow field. If the oscillation and flow reversal are the cause of two/three dimensional flow structures, then it can be assumed that extended oscillation periods can be associated with an extension of the model beyond its principal assumptions.

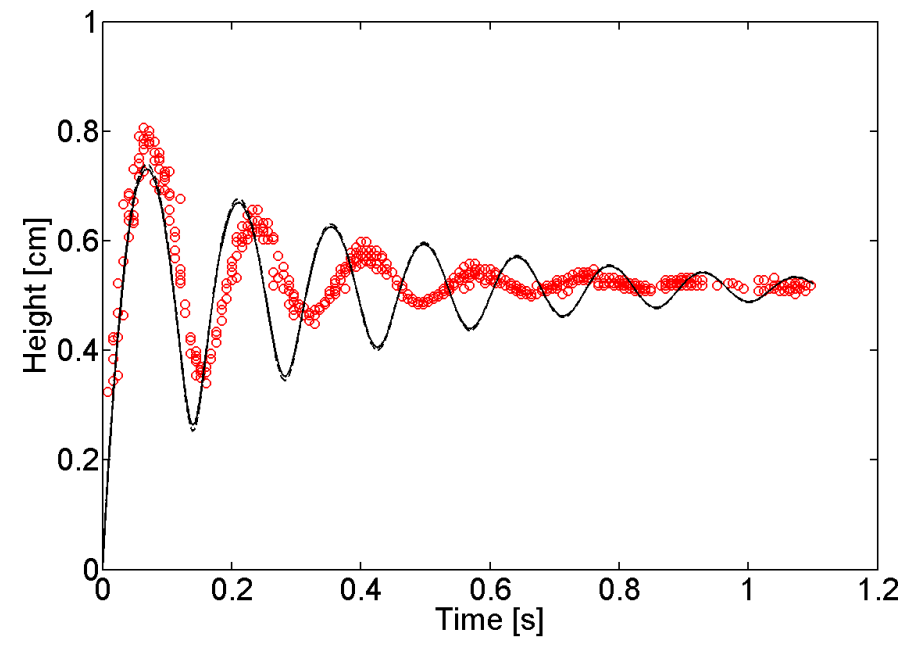

Figure 22: Heated $2.0 \mathrm{~mm}$ capillary rise profiles for n-pentane at $6.0 \mathrm{~W}$.

As the iso-octane is of the same principal structure as n-pentane and is non-polar, a comparison with the model is well suited and should confirm similar trends to those observed for n-pentane. Like the n-pentane cases, the iso-octane profile for the 0.5 mm capillary is observed to follow the early portion and then overpredict the motion due to the assumption that the final height superheat is the same as that in the early stages. 


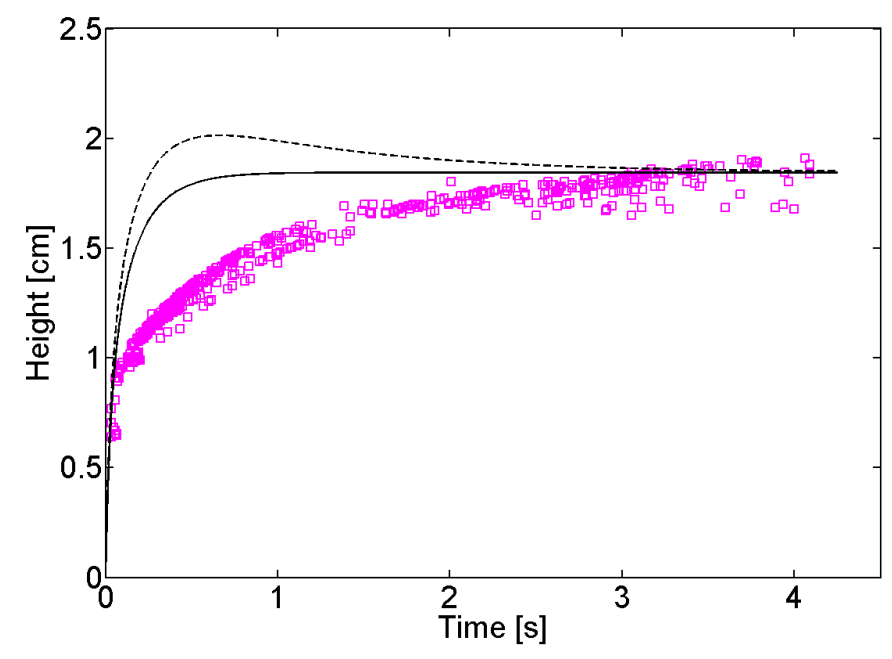

Figure 23: Heated $0.5 \mathrm{~mm}$ capillary rise profiles for iso-octane at $2.7 \mathrm{~W}$.

For the $1.0 \mathrm{~mm}$ and $2.7 \mathrm{~W}$ heated iso-octane case, the oscillations are comparable to the experimental data in both amplitude and phase as illustrated in Figure 24. This trend is consistent with that of the n-pentane case presented previously.

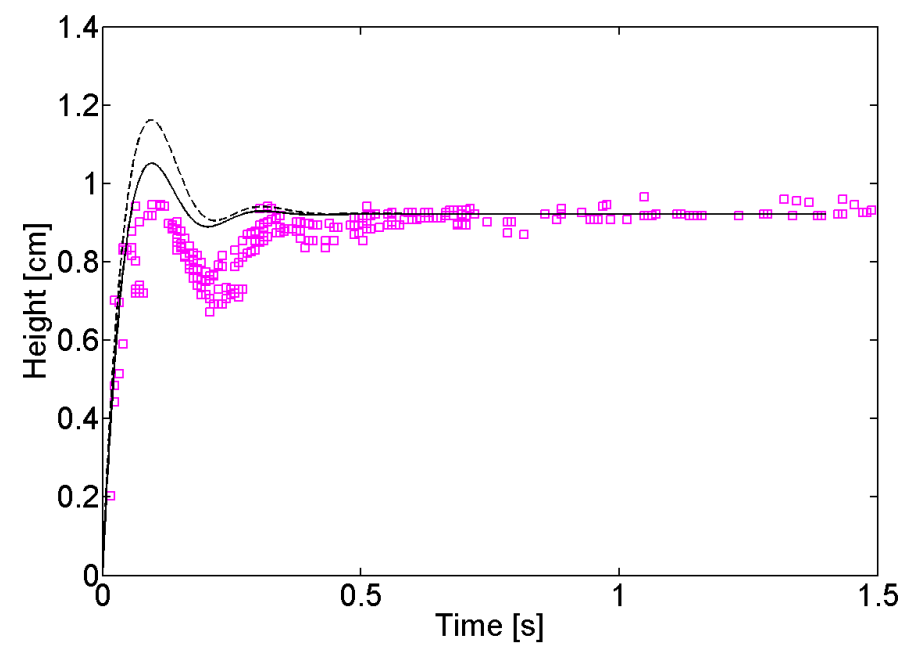

Figure 24: Heated $1.0 \mathrm{~mm}$ capillary rise profiles for iso-octane at $2.7 \mathrm{~W}$.

For the $2.0 \mathrm{~mm}$ and $2.7 \mathrm{~W}$ heated iso-octane cases, the amplitude of the oscillation is close for the range of motion tracked, however the non-linear change in the oscillatory phase becomes apparent with increasing time. This is again consistent with the 
results of n-pentane further supporting the hypothesis that the successive oscillations lead to a divergence between the model assumptions and experimental observations.

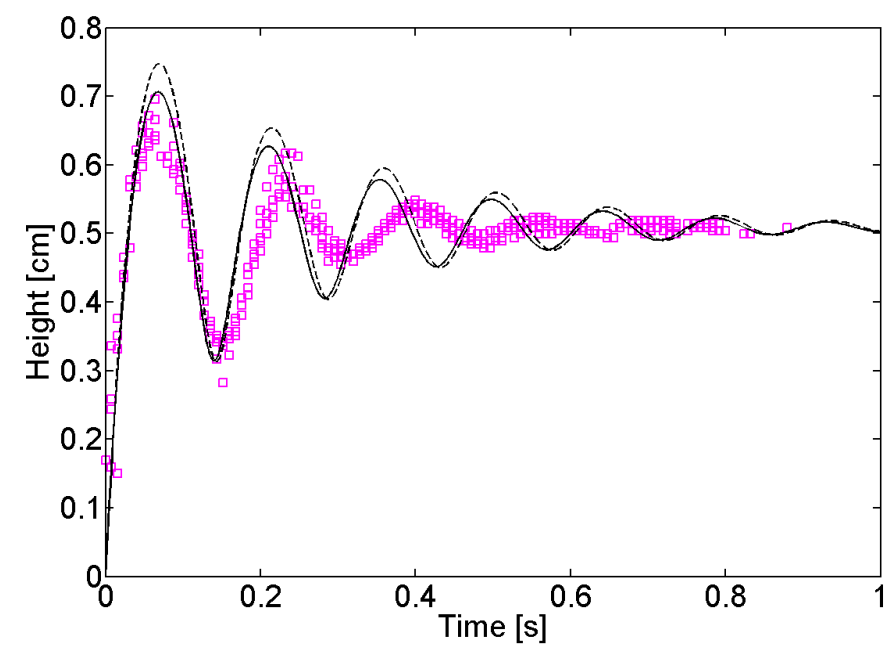

Figure 25: Heated $2.0 \mathrm{~mm}$ capillary rise profiles for iso-octane at $2.7 \mathrm{~W}$.

As the model was developed, there is no immediate cause to assume that the introduction of a mild polarity in the fluid would affect the results. Despite this, the comparison of the three fluids for both unheated and heated cases suggests that there may be some underlying physical mechanisms which are different.

For the acetone experiments, typical cases for a heater setting of $0.7 \mathrm{~W}$ are presented. Once again the $0.5 \mathrm{~mm}$ flow profile differs in the same fashion as for those of n-pentane and iso-octane. This deviation may be once again attributed to the assumption that the equilibrium height and associated superheat are constant throughout the meniscus rise. 


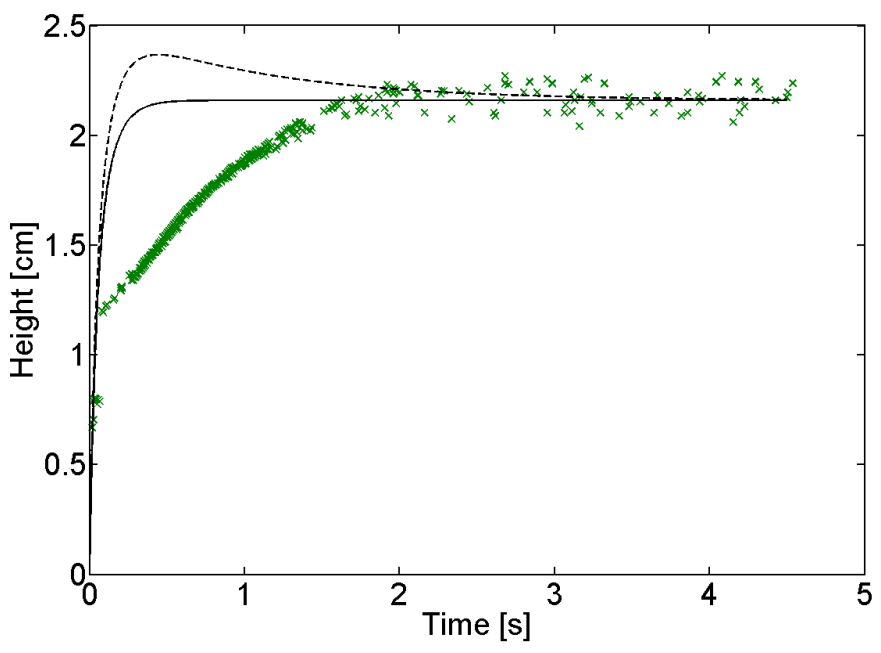

Figure 26: Heated $0.5 \mathrm{~mm}$ capillary rise profiles for acetone at $0.7 \mathrm{~W}$.

When the capillary tube size is increased to $1.0 \mathrm{~mm}$, the oscillation appears to be close in amplitude with a slightly larger amplitude than that of the data. Furthermore, the phase of the oscillation appears similar in the early stages of the motion, but degrades with time.

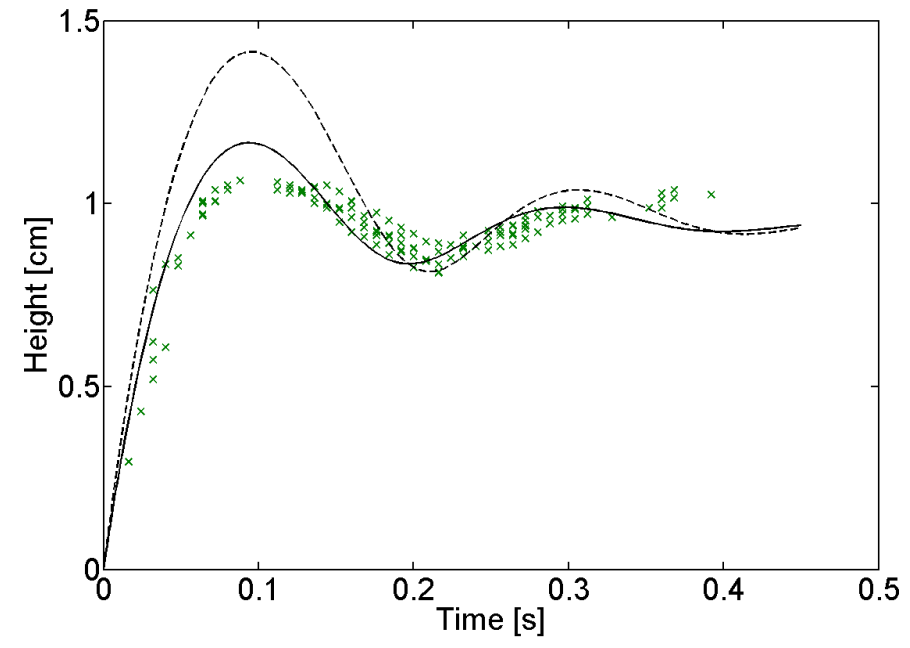

Figure 27: Heated $1.0 \mathrm{~mm}$ capillary rise profiles for acetone at $0.7 \mathrm{~W}$.

The amplitude and phase observed for the $1.0 \mathrm{~mm}$ capillary tubes become amplified in the $2.0 \mathrm{~mm}$ capillary tubes as can be seen when comparing Figures 27 and 28. 
This deviation in the oscillation's phase is consistent with the findings put forth by Zhmud et al. [68] and Lorenceau et al. [81].

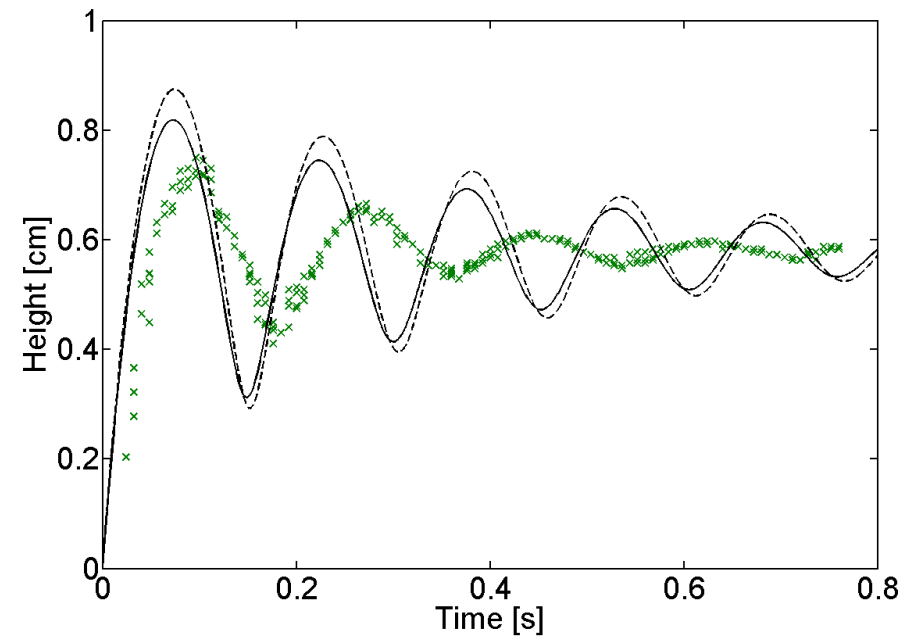

Figure 28: Heated $2.0 \mathrm{~mm}$ capillary rise profiles for acetone at $0.7 \mathrm{~W}$.

From the above comparisons, it is apparent that the model has predictive capabilities, especially in the unheated small capillaries and the non-polar heated 1.0 $\mathrm{mm}$ capillaries. The predictability is noted to degrade as the capillary tube diameter increases and the number of oscillations increases. While the imbibition model addresses many of the flow properties, the non-linear contributions affecting the later stages of the motion are insufficient as evidenced by the differences in the evolution of the oscillation's phase for the $2.0 \mathrm{~mm}$ capillary tube cases.

With regards to the transient evaporation model, its trajectory was always above the constant evaporation rate. This is a consequence of the model as it was proposed on the grounds that the evaporation would asymptotically rise to the rate in the final equilibrium height location. The comparison suggests that the opposite was likely occurring, with the evaporation rate starting high and decreasing toward a constant value as the system comes to rest. 


\subsection{Summary}

A series of experiments were performed for three different working fluids, three capillary tube diameters and five heater settings. The experiments were focused on the meniscus rise dynamics (imbibition) for unheated and heated menisci. The experiments were performed in a controlled environment with the rise dynamics captured using high speed digital photography. The experiments revealed the rise dynamics were dependent upon the fluid, capillary tube size and the applied heat load. The relation between the meniscus height and superheat was verified through the determination of the baseline superheat used to initialise the model. The experimental data was found to be consistent with the trends predicted by the imbibition model, thereby validating the model's application to evaporating meniscus dynamics.

The experimental findings indicated the initial rise of the meniscus was generally consistent with the model for all cases tested. This finding suggests that during the initial rise of the motion the flow can be accurately described as two-dimensional and laminar. When the meniscus is subject to evaporation, the interim dynamics can be approximated to varying degrees; however the model is unable to accurately predict the motion for all cases tested. The experimental results confirmed that evaporation (and any potential transients) can have a measurable effect on the meniscus dynamics and equilibrium height. The transient considerations to the evaporation rate require further refinement. 


\section{Chapter 5}

\section{Meniscus instability - model}

The circumstances surrounding the stability of an evaporating meniscus are of interest to many phase change heat transfer applications. The fundamental physics which govern an evaporating meniscus as discussed previously in Chapter 2 have seen a significant amount of work in describing a stationary evaporating thin-film. The fundamental film stability work of Burelbach et al. [96], laid a solid foundation from which we might expand to the stability of an evaporating meniscus. To this end, it is the objective of this chapter to investigate the potential stability of an evaporating meniscus within the confines of a channel. Let us consider a meniscus contained within a channel with immiscible idealistically smooth parallel walls spaced by a width $W$ as illustrated in Figure 29. 


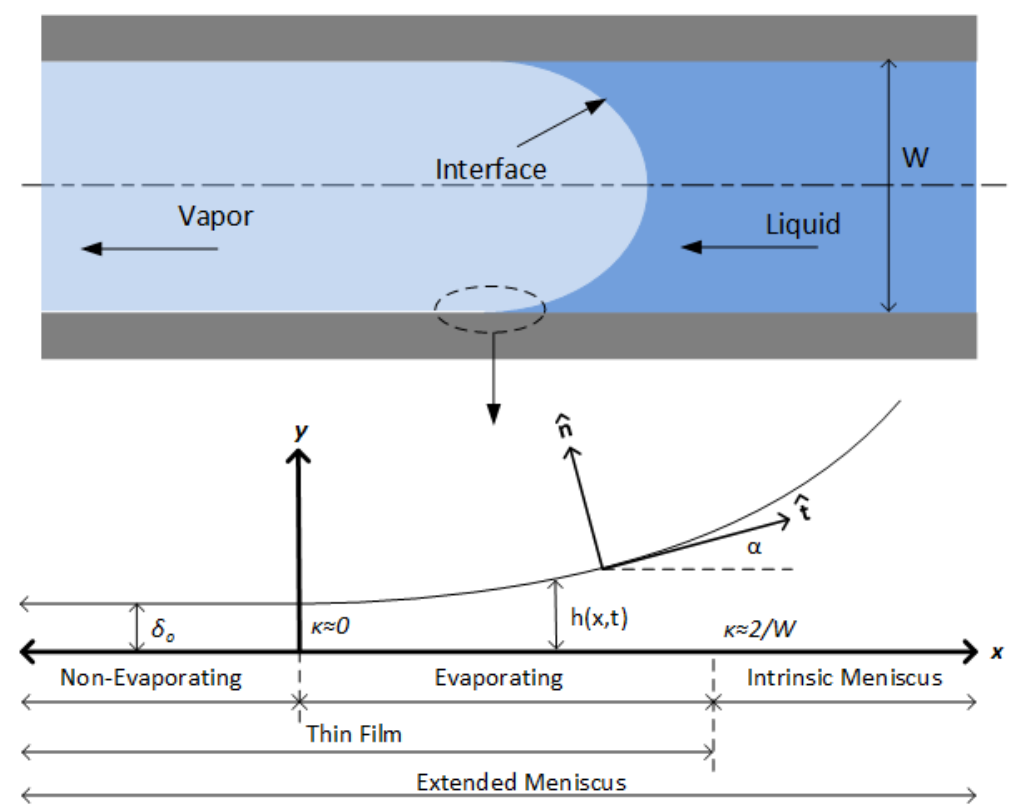

Figure 29: Channel based meniscus and thin-film region.

The channel is assumed to be of infinite length so that end effects can be neglected thereby reducing the problem to that of two-dimensions. The symmetry condition at the centre of the channel is then exploited to reduce the meniscus to that of a monotonically increasing film. To facilitate phase change the channel walls are assumed to be heated and of a constant and uniform wall temperature $T_{w}$. Furthermore, the channel width is limited such that the Bond number is sufficiently small so as to ignore gravitational effects.

While a non-evaporating meniscus can be described by the Young-Laplace equation, the introduction of phase change will lead to changes in film geometry. These changes in geometry lead to the formation of a curved evaporating thin-film asymptotically bounded by a flat and circular sections. Thus, an evaporating meniscus can be constructed into the non-evaporating thin-film (adsorbed region), evaporating thin-film and intrinsic meniscus regions as depicted in Figure 29.

The channel geometry lends itself well to the application of a Cartesian coordinate system, with the origin positioned at the interface of the channel wall and liquid 
and at the transition of the non-evaporating adsorbed region and the evaporating thin-film region. As the liquid-vapour interface is of a curved nature, it is more appropriately described by a normal and tangential coordinate system. Thus, the governing equations will be derived for a Cartesian coordinate system with the normal and tangential system applied when appropriate for the liquid-vapour interface. The normal and tangential unit vectors are related to the Cartesian coordinate system as:

$$
\begin{gathered}
\hat{\mathbf{n}}=\frac{1}{\phi}\left[\begin{array}{c}
-h_{x} \\
1
\end{array}\right] \\
\hat{\mathbf{t}}=\frac{1}{\phi}\left[\begin{array}{l}
1 \\
h_{x}
\end{array}\right]
\end{gathered}
$$

where,

$$
\phi=\sqrt{1+h_{x}^{2}}
$$

With the basis of the problem set, the following sections will provide an in-depth derivation of a linear stability model of an evaporating thin-film. The model is derived in a general sense so that it may be applied to any desired film geometry consistent with the assumptions of the model.

\section{$5.1 \quad$ Governing equations}

The liquid meniscus is assumed to be an incompressible Newtonian fluid and of a sufficient thickness that continuum mechanics hold. The liquid flow is further assumed to be of a laminar nature and of small velocity such that viscous dissipation effects can be ignored. With these assumptions, the two-dimensional continuity, Navier-Stokes 
and the energy equations can be written as:

$$
\begin{gathered}
\nabla \cdot \mathbf{V}=0 \\
\rho\left(\mathbf{V}_{t}+\mathbf{V} \cdot \nabla \mathbf{V}\right)=-\nabla \mathbf{p}+\mu \nabla^{2} \mathbf{V} \\
\rho c_{p}\left(T_{t}+\mathbf{V} \cdot \nabla T\right)=k \nabla^{2} T
\end{gathered}
$$

Two sets of boundary conditions are required for the governing equations. The boundary conditions imposed at the wall $(y=0)$ are:

No slip,

$$
u=0
$$

No penetration,

$$
v=0
$$

Wall temperature,

$$
T=T_{w}
$$

A second set of boundary conditions is needed for the liquid-vapour interface, $y=h(x, t)$. These boundary conditions address the mass and energy jump conditions as well as the surface stresses at the interface. The phase change process at the interface is assumed to occur normal to the interface. Thus, we can write the mass jump balance across the interface as:

$$
j=\rho\left(\mathbf{V}-\mathbf{V}_{l v}\right) \cdot \hat{\mathbf{n}}=\rho_{v}\left(\mathbf{V}_{v}-\mathbf{V}_{l v}\right) \cdot \hat{\mathbf{n}}
$$

The interfacial jump condition must then be transformed such that it is consistent with the Cartesian coordinate system. The liquid and interface velocities can be 
written as:

$$
\begin{gathered}
\mathbf{V}=\left[\begin{array}{ll}
u & v
\end{array}\right] \\
\mathbf{V}_{l v}=\left[\begin{array}{ll}
u_{l v} & v_{l v}
\end{array}\right]
\end{gathered}
$$

The component interface velocities can be related to the Cartesian based local interface velocity and slope as shown in Figure 29. Thus we find the interfacial velocity components are related to the normal and tangential coordinate as:

$$
\begin{gathered}
u_{l v}=-\sin (\alpha) h_{t} \\
v_{l v}=\cos (\alpha) h_{t}
\end{gathered}
$$

where,

$$
\alpha=\arctan \left(h_{x}\right)
$$

Applying the trigonometric and inverse trigonometric relations we find:

$$
\begin{gathered}
u_{l v}=-\frac{h_{x} h_{t}}{\phi} \\
v_{l v}=\frac{h_{t}}{\phi}
\end{gathered}
$$

Substituting Equations (73) and (74) into Equation (67) and solving, we find the evaporative mass flux jump condition for a curved interface as:

$$
j=\frac{\rho\left(v-u h_{x}-\phi h_{t}\right)}{\phi}
$$


The interfacial stress boundary conditions are broken into the normal and tangential stress components. The normal stress boundary condition is then:

$$
j\left(\mathbf{V}-\mathbf{V}_{v}\right) \cdot \hat{\mathbf{n}}+\left(\left(\mathbf{T}-\mathbf{T}_{v}\right) \cdot \hat{\mathbf{n}}\right)^{T} \cdot \hat{\mathbf{n}}-\left(p-p_{v}\right)=p_{c a p}+p_{d}
$$

The capillary pressure is taken to be:

$$
p_{c a p}=\sigma \kappa=\frac{\sigma h_{x x}}{\phi^{3}}
$$

with the surface tension assumed to vary linearly with temperature as:

$$
\sigma=\sigma_{0}-\gamma\left(T_{l v}-T_{r e f}\right)
$$

The disjoining pressure is taken to be for non-polar fluids and of the form:

$$
p_{d}=\frac{A}{h^{3}}
$$

The shear stress at the interface is assumed to have a no slip condition with the Marangoni stress balancing the liquid and vapour stresses and can be written as:

$$
\left(\left(\mathbf{T}-\mathbf{T}_{v}\right) \cdot \hat{\mathbf{n}}\right)^{T} \cdot \hat{\mathbf{t}}=-\nabla \sigma \cdot \hat{\mathbf{t}}
$$

The conservation of energy across the interface is simplified by neglecting the kinetic energy and viscous dissipation effects. Thus we have the simplified energy jump condition as:

$$
j h_{f g}+\left(k \nabla T-k_{v} \nabla T_{v}\right) \cdot \hat{\mathbf{n}}=0
$$

Finally an evaporation model is needed to relate the interfacial conditions with 
the evaporative mass flux. Given the curved nature of the thin-film, the KelvinClapeyron model is most suitable as it addresses the superheat and pressure jump conditions. However, the retention of the Kelvin (curvature) condition makes the governing equations prohibitively complex. Thus, we elect to employ the Clapeyron model for evaporation as:

$$
j=a \Delta T
$$

where,

$$
a=\left(\frac{C^{2} M}{2 \pi R T_{l v}}\right)^{1 / 2}\left(\frac{P_{v} M h_{f g}}{R T_{v} T_{l v}}\right)
$$

The interfacial boundary conditions can be further simplified by a comparative analysis of the phase specific fluid properties. Comparing the vapour and liquid properties of density, viscosity and thermal conductivity, we note:

$$
\begin{aligned}
\frac{\rho_{v}}{\rho} & \ll 1 \\
\frac{\mu_{v}}{\mu} & \ll 1 \\
\frac{k_{v}}{k} & \ll 1
\end{aligned}
$$

Applying the comparative ratios of Equation (84), the two sided model can be reduced to a one sided model. Thus the normal and shear stresses and energy jump boundary conditions simply to:

$$
\begin{gathered}
\frac{j^{2}}{\rho_{v}}+(\mathbf{T} \cdot \hat{\mathbf{n}})^{T} \cdot \hat{\mathbf{n}}-\left(p-p_{v}\right)=\frac{\sigma h_{x x}}{\phi^{3}}+\frac{A}{h^{3}} \\
(\mathbf{T} \cdot \hat{\mathbf{n}}) \cdot \hat{\mathbf{t}}=-\nabla \sigma \cdot \hat{\mathbf{t}} \\
j h_{f g}+k \nabla T \cdot \hat{\mathbf{n}}=0
\end{gathered}
$$




\subsection{Scaling the equations}

The governing equations can be non-dimensionalised using scales pertinent to the problem to achieve a more general form. The scales selected for the nondimensionalisation are in part consistent with those of Benselama et al. [105] and Burelbach et al. [96]. The scaling in the $y$ direction is taken to be the film thickness in the non-evaporating thin-film region (adsorbed film) $y_{c}=\delta_{0}$. The adsorbed thickness is obtained from the Kelvin-Clapeyron evaporation model as: $\delta_{0}=(A b \Delta T / a)^{1 / 3}$. Given the nature of the thin-film, the scales in the $x$ direction are not immediately evident and are therefore left to be determined from other scalings. Thus, we leave the characteristic scale in $x$ as $x_{c}$ which will be defined later. The dimensionless coordinates and film height are taken to be of the forms: $X=x / x_{c}, Y=y / \delta_{0}$ and $\mathcal{H}=h / \delta_{0}$.

The characteristic velocity in the $y$ direction is taken to be the ratio of the evaporative mass flux to liquid density as:

$$
v_{c}=a \Delta T / \rho
$$

Here we elect to assign the characteristic evaporative mass flux as the Clapeyron model for evaporation as given by:

$$
j_{c}=a \Delta T
$$

The characteristic pressure is taken to be the disjoining pressure in the nonevaporating thin-film region $p_{c}=A / \delta_{0}^{3}$ and can be related to the lubrication form of Equation (62) in the $y$ direction yielding:

$$
p_{c}=\frac{\mu u_{c} A}{\delta_{0}^{3}}
$$


The characteristic velocity in the $x$ direction is obtained from continuity, Equation (61). Rearranging, we find the characteristic velocity in $x$ to be:

$$
u_{c}=\frac{A}{\mu \delta_{0}^{2} x_{c}}
$$

Using the characteristic pressure and $\mathrm{x}$-wise velocity the characteristic scale in $x$ is then found as:

$$
x_{c}=\left(\frac{A}{a \Delta T \nu}\right)^{1 / 2}
$$

The time aspect of the governing equations is non-dimensionalised using the viscous time scale as:

$$
t_{c}=\frac{x_{c} \delta_{0}}{\nu}
$$

The non-dimensional time is then taken to be of the form: $\tau=t / t_{c}$.

Finally we non-dimensionalise the temperature using the ratio of temperature differences as:

$$
\theta=\frac{T_{w}-T}{T_{w}-T_{v}}=\frac{T_{w}-T}{\Delta T}
$$

Applying the characteristic scales to the continuity, momentum and energy equations, Equation (61)-(63), we obtain their non-dimensional forms as:

$$
\begin{gathered}
U_{X}+V_{Y}=0 \\
\operatorname{Re} \zeta\left(U U_{X}+V U_{Y}\right)=-P_{X}+\zeta^{2} U_{X X}+U_{Y Y} \\
R e \zeta^{3}\left(U V_{X}+V V_{Y}\right)=-P_{Y}+\zeta^{2}\left(\zeta^{2} V_{X X}+V_{Y Y}\right) \\
P e \zeta^{2}\left(U \theta_{X}+V \theta_{Y}\right)=\zeta^{2} \theta_{X X}+\theta_{Y Y}
\end{gathered}
$$

where $\zeta=y_{c} / x_{c}$ is the characteristic ratio of the $x$ and $y$ scalings. Given the scales selected, the characteristic ratio is a small quantity such that $\zeta \ll 1$. 
Here we define the Reynolds $(R e)$ and Peclet $(P e)$ numbers as:

$$
\begin{gathered}
R e=\frac{\rho u_{c} \delta_{0}}{\mu}=\frac{a \Delta T x_{c}}{\mu} \\
P e=\frac{\rho c_{p} u_{c} x_{c}}{k}=\frac{c_{p} a \Delta T x_{c}^{2}}{\delta_{0} k}
\end{gathered}
$$

Next the boundary conditions of the wall $Y=0$ are non-dimensionalised giving:

$$
\begin{aligned}
& U=0 \\
& V=0 \\
& \theta=0
\end{aligned}
$$

To scale the boundary conditions at the interface the non-dimensional $Y$ coordinate is assigned as: $Y=\mathcal{H}(X, \tau)$. The scaled interfacial evaporative, normal and shear stresses and energy jump conditions, Equations (75) and (85)-(87), at the liquid-vapour interface are then:

$$
\begin{gathered}
J=\frac{R e\left(V-\mathcal{H}_{X} U\right)-\Phi \mathcal{H}_{\tau}}{R e \Phi} \\
J^{2} R e_{m} \zeta^{2}-\frac{2 \zeta^{2}\left(\zeta^{2} \mathcal{H}_{X}\left(V_{X}-\mathcal{H}_{X} U_{X}\right)+\mathcal{H}_{X} U_{Y}+U_{X}\right)}{\Phi^{2}} \\
-\left(P-P_{v}\right)=\frac{\zeta^{4} \mathcal{H}_{X X}}{C a \Phi^{3}}+\frac{\Gamma \zeta}{\operatorname{Re} \mathcal{H}^{3}} \\
P e U_{Y}\left(\Phi^{2}-2\right)+P e \zeta^{2}\left(4 \mathcal{H}_{X} U_{X}+V_{X}\left(\Phi^{2}-2\right)\right)=M a \zeta \Phi\left(\mathcal{H}_{X} \theta_{Y}+\theta_{X}\right) \\
J E \Phi-\theta_{Y}+\zeta^{2} \mathcal{H}_{X} \theta_{X}=0
\end{gathered}
$$


Where the dimensionless numbers are given as:

$$
\begin{gathered}
\Phi=\sqrt{1+\left(\zeta \mathcal{H}_{X}\right)^{2}} \\
R e_{m}=\frac{\delta_{0} v_{c} \rho}{\nu \rho_{v}} \\
C a=\frac{\mu a \Delta T}{\rho \sigma_{0}} \\
\Gamma=\frac{\rho A}{\mu^{2} \delta_{0}} \\
M a=\frac{\gamma \Delta T x_{c} \rho c_{p}}{\mu k} \\
E=\frac{\delta_{0} a h_{f g}}{k}
\end{gathered}
$$

Finally the Clapeyron evaporation model, Equation (82), is scaled yielding:

$$
J=1-\theta
$$

\subsubsection{Reduction of the characteristic ratio}

The characteristic ratio $(\zeta)$ appears throughout the governing equations and associated boundary conditions. As noted previously, this characteristic ratio is found to be significantly less than unity $(\zeta \ll 1)$. Therefore we can apply this condition to the governing equations to obtain the leading order governing equations as:

$$
\begin{gathered}
U_{X}+V_{Y}=0 \\
P_{X}=U_{Y Y} \\
P_{Y}=0
\end{gathered}
$$




$$
\theta_{Y Y}=0
$$

While the same simplification can be extended to the jump conditions at the interface, it's application can lead to an oversimplification of the normal stress, shear stress and energy boundary conditions. Given the purpose of this study is to investigate how the vapour recoil, surface tension, long-range molecular forces and thermocapilliarity contribute to thin-film stability, these terms are actively retained. The leading-order interfacial boundary conditions are then:

$$
\begin{gathered}
J^{2} R e_{m} \zeta^{2}-\left(P-P_{v}\right)=\frac{\zeta^{4} \mathcal{H}_{X X}}{C a \Phi^{3}}+\frac{\Gamma \zeta}{R e \mathcal{H}^{3}} \\
P e U_{Y}\left(\Phi^{2}-2\right)=\operatorname{Ma} \zeta \Phi\left(\mathcal{H}_{X} \theta_{Y}+\theta_{X}\right) \\
J E \Phi-\theta_{Y}=0
\end{gathered}
$$

The leading-order forms of the mass jump, Equation (104), and Clapeyron evaporation model, Equation (114) remain unchanged.

\subsection{Evolution equation}

The system of leading-order governing equations along with the associated boundary conditions are solved to obtain a single evolution equation. The result will describe the evolution of an evaporating thin-film. To begin, we integrate the energy equation, Equation (118), twice and apply the wall and interface boundary conditions, Equations (103) and (121) respectively. The resulting dimensionless temperature is:

$$
\theta=E J \Phi Y
$$

We can then substitute the non-dimensional mass flux relation from Equation 
(114) into Equation (122) and evaluate at the interface $Y=\mathcal{H}(X, \tau)$. Thus we find the dimensionless mass flux to be:

$$
J=\frac{1}{E \Phi \mathcal{H}+1}
$$

Back substituting Equation (123) into Equation (122) we obtain the dimensionless temperature:

$$
\theta=\frac{E \Phi Y}{E \Phi \mathcal{H}+1}
$$

Next, we integrate Equation (117) and apply the interfacial jump boundary condition, Equation (119). Substituting Equation (123) for the dimensionless mass flux, we obtain:

$$
P=P_{v}+\frac{R e_{m} \zeta^{2}}{(E \Phi \mathcal{H}+1)^{2}}-\frac{\zeta^{4} \mathcal{H}_{X X}}{C a \Phi^{3}}-\frac{\Gamma \zeta}{R e \mathcal{H}^{3}}
$$

Having obtained the liquid pressure in the film we can differentiate Equation (125) in the $\mathrm{X}$ direction and substitute the result into Equation (116). The resulting differential equation is that of a second order differential for the velocity in the Ydirection. Collecting the terms on the right hand side, we can redefine the right hand side as:

$$
\Lambda=\frac{\zeta^{4}\left(3 \Phi_{X} \mathcal{H}_{X X}-\Phi \mathcal{H}_{X X X}\right)}{C a \Phi^{4}}-\frac{2 R e_{m} \zeta^{2}\left(E \Phi \mathcal{H}_{X}+E \Phi_{X} \mathcal{H}\right)}{(E \Phi \mathcal{H}+1)^{3}}+\frac{3 \Gamma \zeta \mathcal{H}_{X}}{R e \mathcal{H}^{4}}
$$

Thus, we find Navier-Stokes in $Y$ to be:

$$
U_{Y Y}=\Lambda
$$

Integrating Equation (127) twice in $Y$, subject to the no slip, Equation (101), and 
the shear stress jump condition, Equation (120), the liquid velocity is:

$$
U=\frac{\left(2 E M a \zeta \Phi \Phi_{X}\left(1-E \Phi^{2} \mathcal{H}_{X}\right)-\Lambda\right) Y^{2}+E M a \zeta \Phi^{2} \mathcal{H}_{X}(E \Phi \mathcal{H}+1) Y}{\operatorname{Pe}\left(\Phi^{2}-2\right)(E \Phi \mathcal{H}+1)^{2}}
$$

We can now solve continuity, Equation (95), by differentiating Equation (128) in $X$ and substituting the result into Equation (95). Integrating the result once with respect to $Y$ we obtain the dimensionless liquid velocity $\mathrm{V}$ as:

$$
V=V_{1} Y^{2}+V_{2} Y^{3}
$$

with the $V_{1}$ and $V_{2}$ coefficients defined in Appendix A.

Having solved the velocity normal to the wall, we can now solve the mass jump boundary condition at the interface, Equation (104). Substituting Equations (123), (126) - (129) into Equation (104), we obtain the leading-order evolution equation as given in Appendix B.

The evolution equation contains $\Phi(X, \tau)$ which is a function of space and time as denoted in Equation (108). As both the interfacial slope and characteristic ratio are assumed to be small, we can make the following approximation:

$$
\Phi(X, \tau) \approx 1
$$

Substituting Equation (130) into Equation (151), we get a simplified form of the leading-order evolution equation as given in Appendix C. The resulting non-linear differential equation describes the evolution of an evaporating liquid interface.

\subsubsection{Linear stability model}

To investigate the stability of an evaporating film we must apply a perturbation to the simplified evolution equation. Thus we perturb the base state film thickness $H$ 
by a small perturbation $h_{p}$ as:

$$
\mathcal{H}(X, \tau)=H(X, \tau)+h_{p}(X, \tau)
$$

where the perturbation is assumed to be of the form:

$$
h_{p}(X, \tau)=\delta H(\tau) \times \exp (i k X)
$$

Substituting Equation (131) into Equation (152) we obtain the perturbed form of the simplified evolution equation. The base state of the result can be removed using a pair of binomial series:

$$
\begin{gathered}
\left(1+\frac{E h_{p}}{E H+1}\right)^{-m}=1-\left(1+\frac{E h_{p}}{E H+1}\right)^{-m}+O(2) \\
\left(1+\frac{h_{p}}{H}\right)^{-n}=1-\left(1+\frac{h_{p}}{H}\right)^{-n}+O(2)
\end{gathered}
$$

Truncating the higher order terms for the binomial series, we can remove the base state, Equation (152), from the perturbed evolution equation. As we are concerned with physical manifestation of the perturbation we simplify the result by dropping the imaginary terms. Thus, we obtain a leading-order perturbation evolution equation of the form:

$$
\frac{h_{p_{\tau}}}{h_{p}}=\mathcal{A} k^{4}+\mathcal{B} k^{2}+\mathcal{C}
$$

with the full form provided in Appendix D. Equation (135) describes the growth rate of a surface perturbation as a function of a disturbance wavenumber $k$. From Equation (135) it can be deduced that for a given disturbance wavenumber, fluid properties and liquid film geometry, the perturbation may grow (instability) or decay (stability). 


\section{$5.4 \quad$ Film stability analysis}

To investigate the stability of an evaporating liquid film we must first make a few assumptions. As it was previously assumed, the working fluid is a single pure species, and for the purposes of this analysis selected to be n-pentane with temperature dependent fluid properties.

In addition to the fluid properties, a base state for the evaporating liquid film is needed. While the transient aspect of Equation (152) can be removed, no closed form solution is readily available. To address this issue we look at the composition of a meniscus. As depicted previously in Figure 29, an evaporating thin liquid film can be broken into three distinct regions. Thus, we begin the analysis with the nonevaporating thin-film region as is similar to that done previously by Burelbach et al. [96].

\subsubsection{Flat film}

The thin-film base state is herein taken to be flat and parallel to the wall. This quasiparallel interfacial stability theory has been used in previous works such as Unsal [117] and Pratt et al. [109]. For an evaporating flat thin-film to remain at steady state, a resupply of fluid into the thin-film is needed to compensate that which has been lost to evaporation. Thus, the flat base state is of the following form:

$$
\begin{gathered}
H=1 \\
H_{X}=H_{X X}=H_{X X X}=H_{X X X X}=0
\end{gathered}
$$

Substituting the base state conditions, Equations (136)-(137), into Equation (135) we obtain the coefficients as:

$$
\mathcal{A}=\frac{\zeta^{4} R e}{6 C a}
$$




$$
\begin{gathered}
\mathcal{B}=\frac{\zeta E^{2}(-3 P e \Gamma(E+3)-\operatorname{MaRe}(E+4))}{6 P e(E+1)^{3}} \\
+\frac{\zeta E\left(2 P e R e R e_{m} \zeta-9 P e \Gamma-3 M a R e\right)-3 P e \Gamma \zeta}{6 P e(E+1)^{3}} \\
\mathcal{C}=\frac{E R e}{(E+1)^{2}}
\end{gathered}
$$

As the closed form base case is simply set to unity with the derivatives being zero, the perturbation evolution equation coefficients are independent of space. Thus, the stability of a flat film is strictly a function of the perturbation (disturbance) wavenumber $(k)$ and the applied superheat $(\Delta T)$.

The superheat affects on film stability are contained within the adsorbed film thickness residing within the non-dimensional numbers. This quantity originates from the Kelvin-Clapeyron evaporation model, where the disjoining pressure and capillary pressure are clearly defined, yielding a film thickness as a function of the applied superheat as previously demonstrated by Polansky [29].

Looking at the film stability based on the wavenumber it is noted that the perturbation grows for wavenumbers of zero as given by $\mathcal{C}$. This suggests that an evaporating flat liquid film is naturally unstable. This type of instability has been noted in previous works such as Burelbach et al. [96] and Sheludko [97]. This result is clearly seen in Figure 30, where the growth rate is plotted against the wavenumber for a range of superheats. 


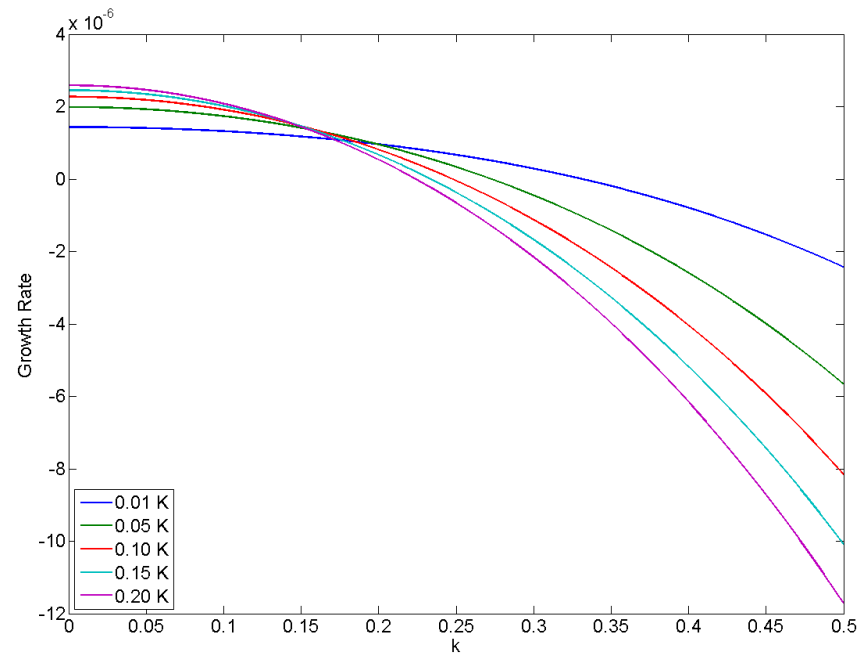

Figure 30: Perturbation growth rates for an evaporating flat film.

The perturbation growth rate is observed to start positive and decrease toward negative values with increasing perturbation wavenumbers. Thus, for a given superheat there exists a critical wavenumber $\left(k_{c}\right)$, where the growth rate is zero. For wavenumbers greater than $k_{c}$, the perturbation decays and the film is stable.

As the superheat is increased, the film thickness decreases and the evaporative mass flux increases. These changes lead to increases in the magnitudes of growth rate in the unstable and stable wavenumber regions. It is also noted that the critical wavenumber is found to decrease with increasing superheat values.

\subsubsection{Modified Young-Laplace}

While previous works have utilised the flat film analysis as a basis for evaporating meniscus stability, it is only valid for a narrow region of the meniscus. The majority of an evaporating meniscus is comprised of the intrinsic meniscus. For a meniscus in equilibrium, the interface is naturally stable. Thus it can be speculated that the intrinsic region of an evaporating meniscus would exhibit similar tendencies. To investigate this, a modified form of the Young-Laplace geometry can be used as a 
base state for the perturbation growth rate equation. To make the analysis closer to that of an evaporating case, the Young-Laplace circular section is shifted to account for the adsorbed film thickness, which as mentioned before, is a function of superheat.

The modified Young-Laplace (MYL) base state is taken to be the segment of a circle with the centre positioned at the symmetry plane of the channel. The radius of the circular section is taken to be half of the channel width less the adsorbed thickness. Thus the closed form of the MYL base state is then:

$$
H=\frac{W / 2+\delta_{0}-\sqrt{(W / 2)^{2}-\left(X x_{c}\right)^{2}}}{\delta_{0}}
$$

As Equation (141) is a function of space and is continuously differentiable over the region of interest, the resulting stability analysis becomes a function of space, wavenumber and superheat. Once again, the superheat is contained within the adsorbed film thickness. Drawing on the results of the flat film base case's variance in superheat, the results here are expected to be principally driven by the geometry and wavenumber.

Substituting Equation (141) into Equation (135) we obtain the perturbation growth rate equation for a MYL base state. The stability of a MYL geometry interface is first studied by first looking at a simple case for a given channel width $(W=10$ $\mu \mathrm{m})$, superheat $(\Delta T=0.05 \mathrm{~K})$ and perturbation wavenumber $(k=0.5)$ as shown in Figure 31. 


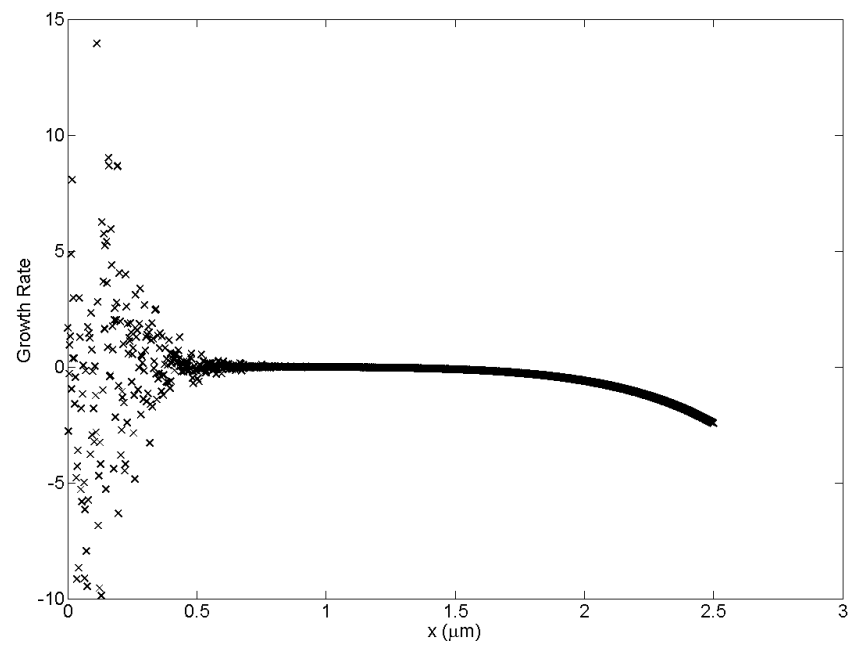

Figure 31: Perturbation growth rates for an evaporating MYL film.

The extension of the leading-order perturbation growth rate equation remains subject to the geometric assumption previously placed as $\Phi \approx 1$. This assumption was based on the assumption that $\zeta H_{X} \ll 1$. For the MYL case, this assumption becomes invalid as $x \rightarrow W / 2$, where $H_{X} \rightarrow \infty$. However for the unique case of circle, the slope is slow to rise such that the assumption holds out to approximately $90 \%$ of the channel width where $\zeta H_{X}=O(-2)$.

Thus, the results shown in Figure 31 are consistent with the assumptions previously made. From the results of Figure 31, it can be seen that the intrinsic region of the meniscus is inherently stable as one might expect. This result is favourable as the evaporation rates in this region are believed to taper off thereby satisfying the Young-Laplace condition imposed. Moving closer to the evaporating thin-film region the perturbation growth rate begins to show signs of instability. The growth rate begins to switch between positive and negative values in a seemingly chaotic fashion. Given the MYL base case asymptotically tends to a slope of zero near the non-evaporating thin-film region, it may be expected that the perturbation growth rate should behave similar to that of the flat film case. 
While a positive growth rate in this region is sufficient basis to determine if the film can be destabilised, it is worth looking at how the growth rate is related to the perturbation wavenumber. Thus, we can plot the growth rate three dimensionally as shown in Figure 32.

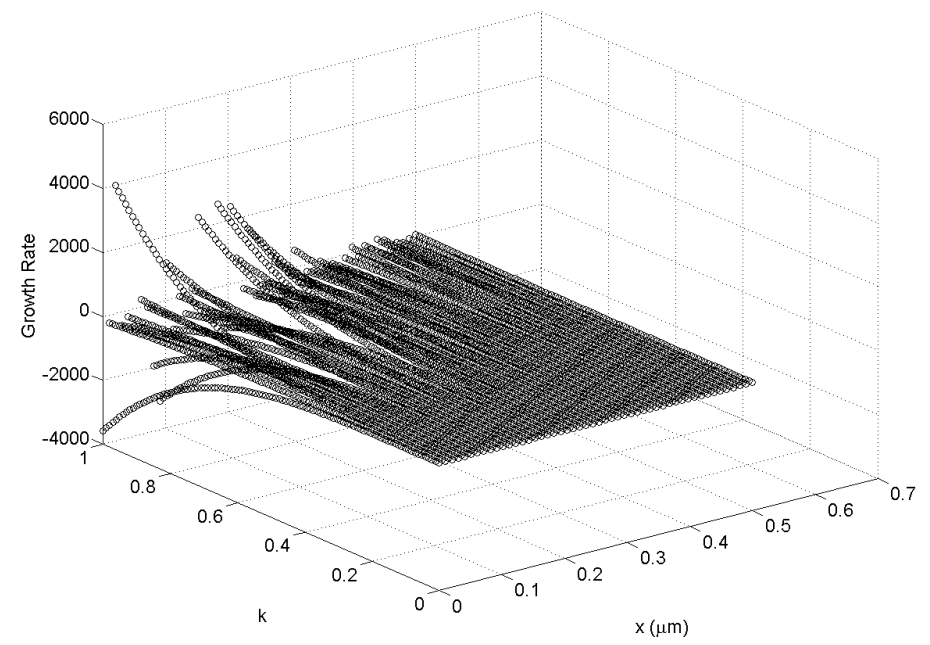

Figure 32: 3-D Perturbation growth rates for the first 10\% of an evaporating MYL film.

From Figure 32, it is clear that the dispersion of the perturbation growth rate is a function of the perturbation wavenumber. The sign of the growth rate as observed in Figure 31 demonstrates the unpredictability of the growth rate. However, it is apparent that the simple cross-section is only one small piece. The chaotic nature is still present, but it is seen that each point corresponds to its own unique trajectory.

Figure 33 shows the side view of the three dimensional plot. The sporadic solutions observed in Figure 31 is a bisection of the data and fails to capture the solutions dependence on the perturbation wavenumber. Here it can be clearly seen that positional dependence still generates a sporadic set of solutions. 


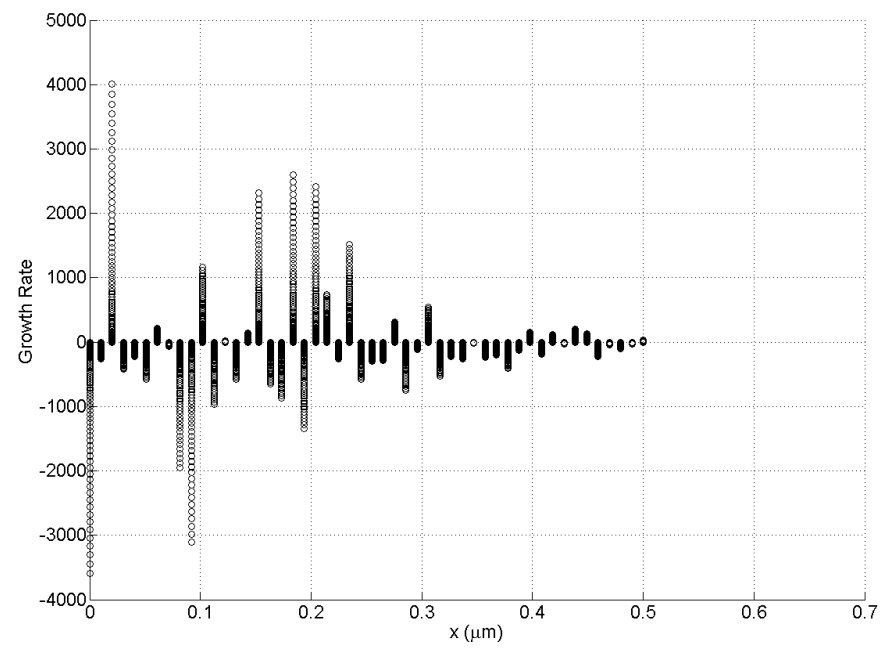

Figure 33: Side view of the 3-D perturbation illustrating the growth rates sporadic tendency.

Looking at the wavenumber and growth rates, the trend becomes clear. Each positional solution, that deviates from the main line follows a diverging trajectory for both stable and unstable growth rates.

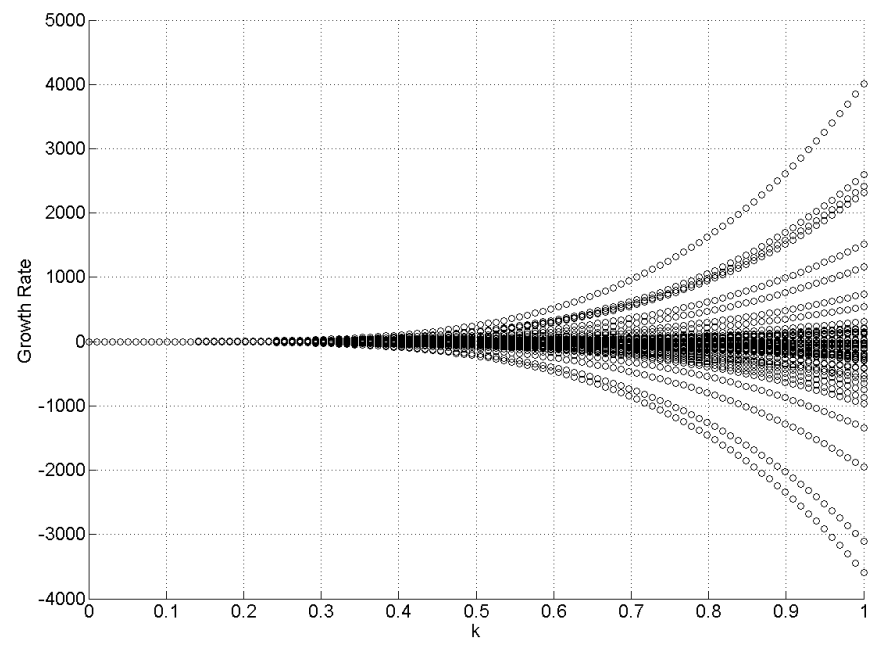

Figure 34: Side view of the 3-D perturbation illustrating the trajectories of the seemingly sporadic points. 
The introduction of a curved interface is the first introduction of a spatial component to the study of evaporating thin-film stability. The application of a circular base state provides a clean mathematical representation of the interface and allows for a detailed investigation of the solutions.

As can be deduced from the above MYL analysis, the intrinsic region of an evaporating meniscus is expected to be inherently stable. As we shift along the meniscus, the perturbation growth rate tends toward a zero growth rate condition and eventually becomes sporadic in nature. The sporadic perturbation growth rate values in the evaporating thin-film region can be stabilising or destabilising. This confirms that the evaporating thin-film region is likely the source of instability and requires further investigation.

\subsubsection{Curved thin-film base state}

The theoretical study of an evaporating meniscus has been pursued extensively as outlined in Chapter 2, with many works focusing on the properties of the evaporating thin-film $[3,7,9,15,17,18,24]$. Of the many models describing the evaporating thinfilm, much of the work has converged toward a third order non-linear differential equation describing a steady state evaporating thin-film. The curved thin-film model developed by Polansky [29] is used as a baseline for obtaining the thin-film properties.

The development of the model and its method of solving are outlined in $[23,29,33]$. Briefly, the thin-film model used herein has no closed form solution. Therefore, the thin-film model must be solved using a Runge-Kutta numerical method. The model is initialised using a set of perturbations. A shooting method is employed on the first derivative and the initial conditions sought to achieve the far field curvature condition of $2 / W$ within a tolerance of $\pm 0.02 / W$. The solutions generated are of a dimensional nature and are non-dimensionalised using the characteristic scales outlined previously.

The nature of the thin-film model is such that it is dependent upon the superheat 
and geometry. Thus, the thin-film geometry differs for each superheat and must be solved for a range of superheats for a given channel geometry. Furthermore, the sensitivity of the model leads to limitations in the solutions, such that as the superheat increases, the model requires greater computational precision. Thus, the thin-film model can only be solved for a limited range of superheats. A sample set of thin-film solutions are presented in Figures 35 - 38.

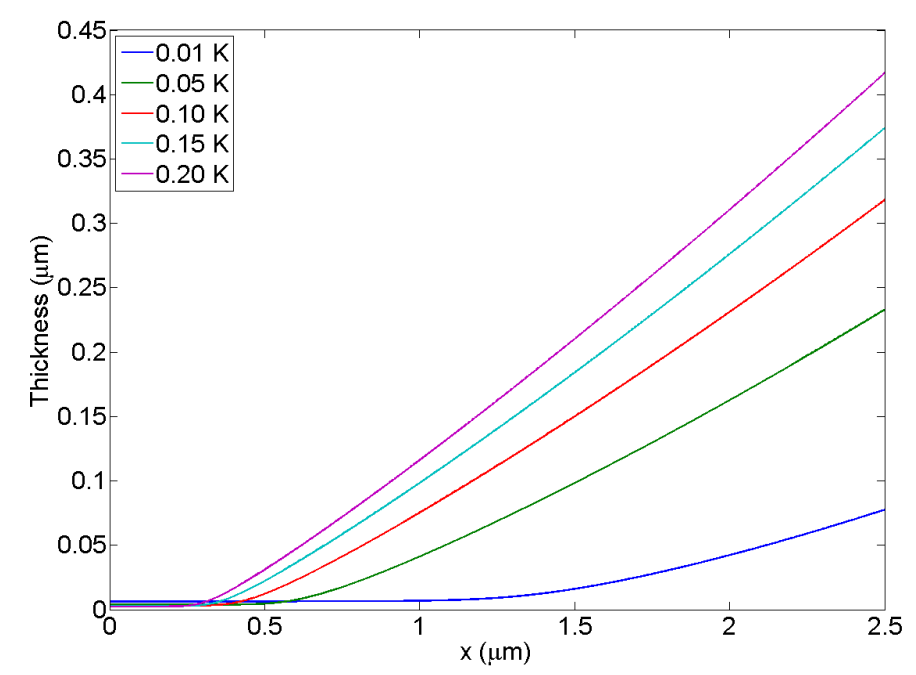

Figure 35: Thin-film thickness for a range of superheats.

As illustrated in Figure 35, the thin-film is notably sensitive to the applied superheat. With increasing superheat, the thin-film is noted to steepen and transition sooner with the thin-film region narrowing. To ensure the stability model is valid for the thin-film solutions, the slope is checked against the assumption $\zeta H_{X} \ll 1$, and found to hold for all cases tested. As the thin-film thickness changes with the applied superheat, the higher order derivatives change in accordance with the thin-film model. 


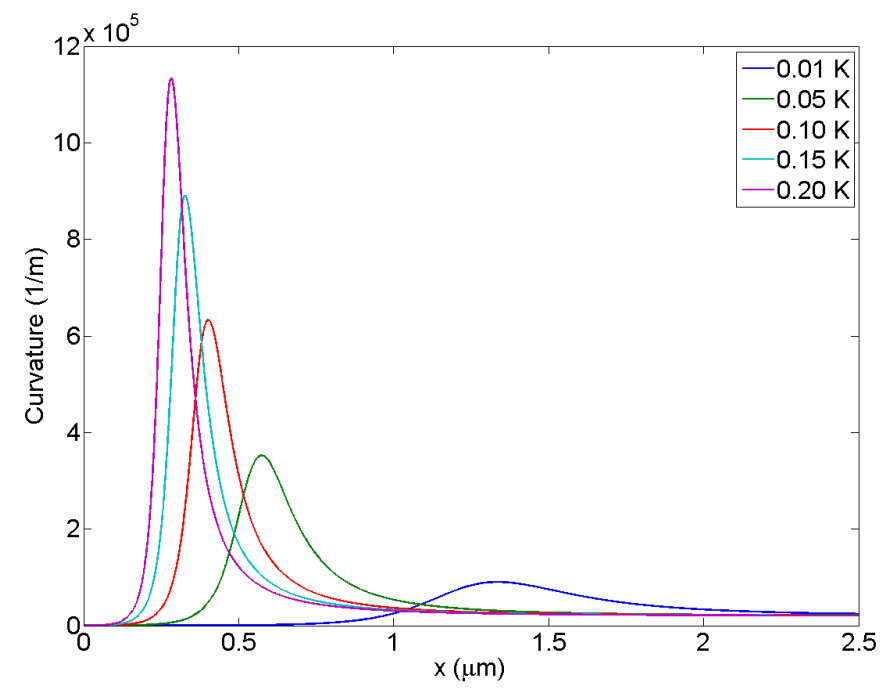

Figure 36: Thin-film curvature for a range of superheats.

With exceptionally small superheats, the local curvature is noted to increase asymptotically toward the Young-Laplace far field curvature condition as noted by Ball et al. [33]. As the superheat is increased, the thin-film model predicts an overshoot of the local curvature. This overshoot condition was experimentally observed in the work of Panchamgam et al. [24]. It may also be noted that with increasing superheat the local curvature overshoot increases in magnitude and shifts spatially as illustrated in Figure 36. 


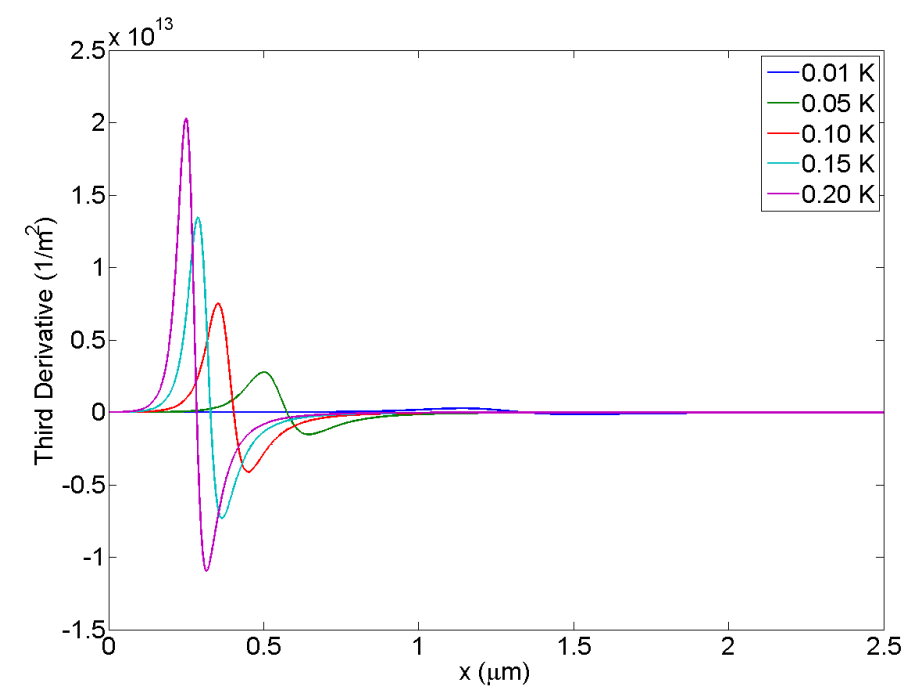

Figure 37: Thin-film third derivative for a range of superheats.

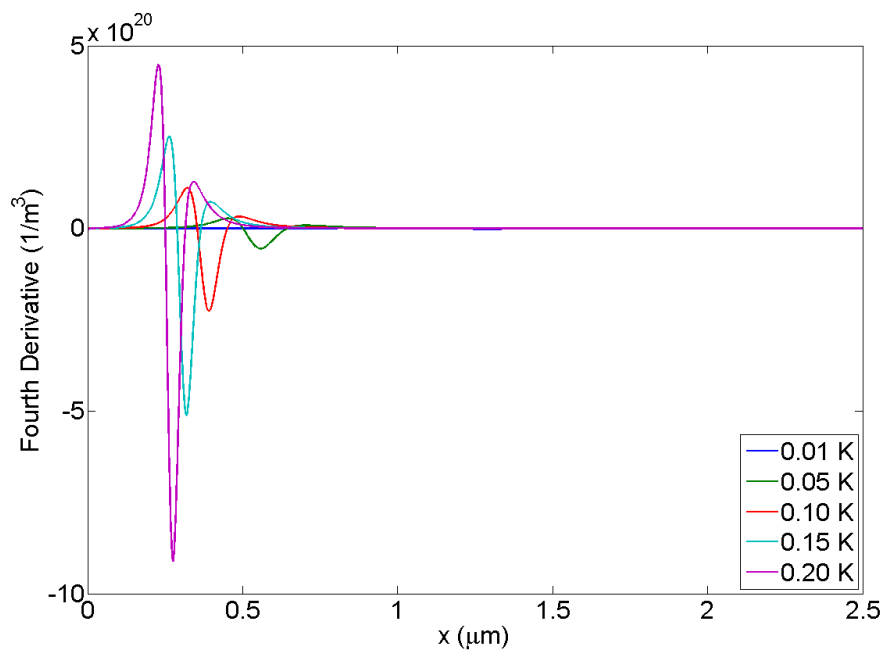

Figure 38: Thin-film fourth derivative for a range of superheats.

Accompanying the curvature overshoot is a change in the higher order derivatives ( $H_{X X X}$ and $\left.H_{X X X X}\right)$ as is consistent with the thin-film model. Such changes in curvature lead to more complex higher order derivatives than those of the MYL model. The spatial changes in sign of the higher order derivatives will inevitably result in differences in the stability of the thin-film as they are carried in the perturbation growth rate coefficient $\mathcal{C}$ of Equation (135). Given the consistency in the numerical 
results with that of the flat base case MYL base case and the experimental study of evaporating thin-films, the thin-film model utilised in this study is expected to result in a representative study of thin-film stability.

\subsubsection{Thin-film stability}

As the thin-film model can be solved for a range of channels, fluids and superheats it is possible to use the geometry information to seed the stability model as described by Equation (135). As the thin-film model can only be solved numerically, the geometric information of the thin-film is limited to the spatial resolution of the step size $(1 \mathrm{~nm})$ used in solving the thin-film model.

For example, let's determine the stability characteristics for n-pentane over a range of superheats in a $100 \mu \mathrm{m}$ wide channel. Given the form of Equation (135), it is possible to map out the cutoff wavenumbers and determine the transitions in thin-film stability as illustrated in Figure 39.

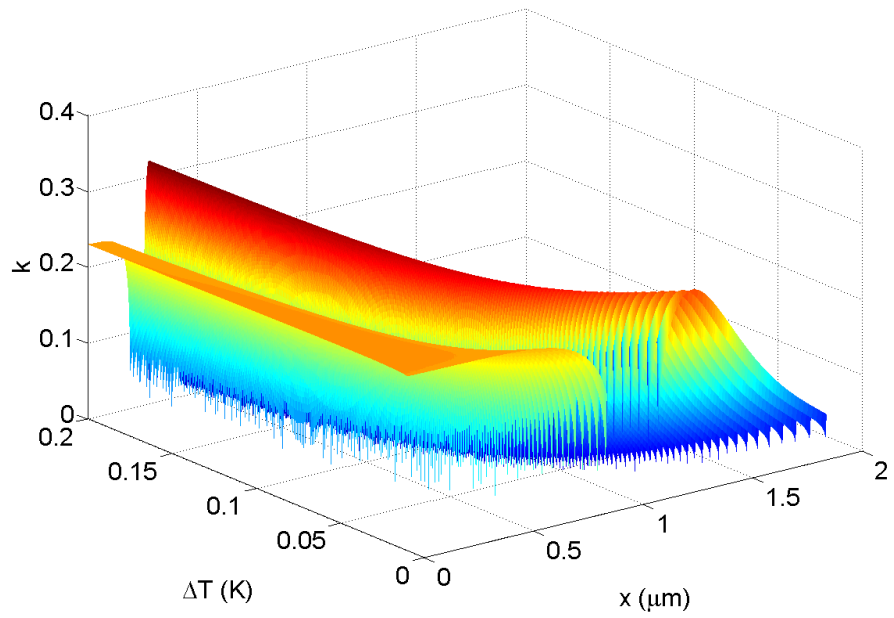

Figure 39: Cutoff wavenumbers for a $100 \mu \mathrm{m}$ channel and n-pentane for a range of superheats.

Figure 39 shows the cutoff wavenumber surface, for which any points above the 
surface indicate stability, while points below indicate instability. The broken nature of the surface as $k_{c} \rightarrow 0$ is a consequence of the spatial resolution of the thin-film model solutions and the cutoff wavenumber gradients. Given the gradients and broken nature, alternate perspectives of the surface are presented in Figures 40 - 42.

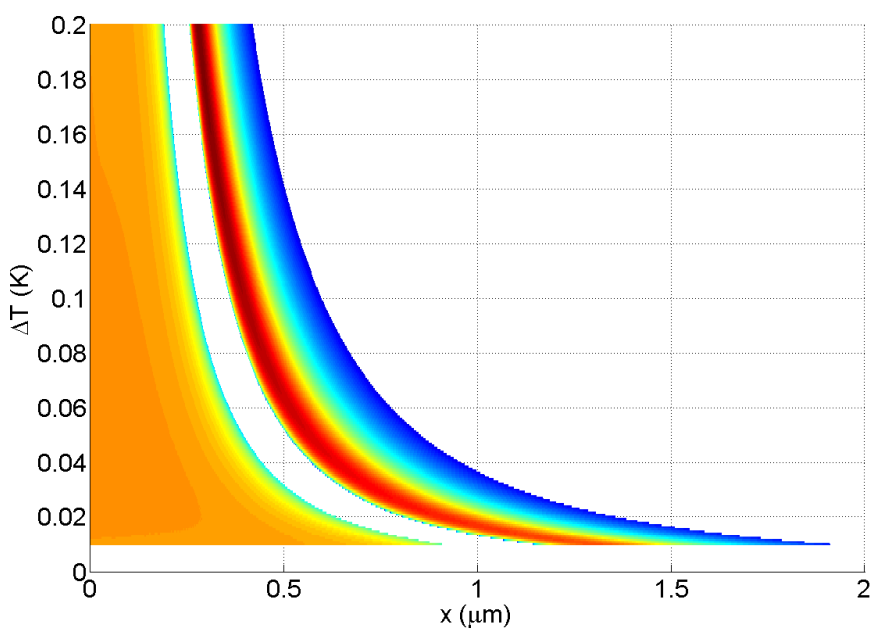

Figure 40: Cutoff wavenumbers based on $\Delta T$ and $x$.

As can be seen from Figure 40, the thin-film cutoff wavenumbers are confined to two spatial regions. The separation of the two spatially dependent stability regions is noted to shift spatially with superheat. As the superheat increases, the stability region shifts toward the non-evaporating thin-film region. This shift is accompanied by a spatial reduction of the unstable regions. 


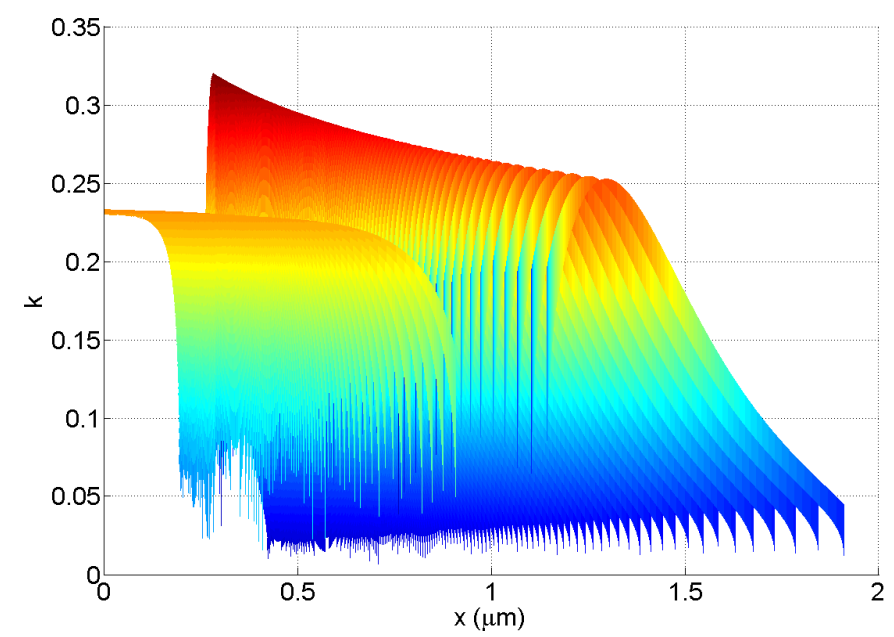

Figure 41: Cutoff wavenumbers based on $k$ and $x$.

The spatial shift is also accompanied by a change in the range of wavenumbers able to destabilise the thin-film as observed in Figure 41. The unstable region nearest the non-evaporating thin-film region shows little change in the wavenumbers and superheat, however the second instability region shows a dependence upon superheat, as can be better seen in Figure 42.

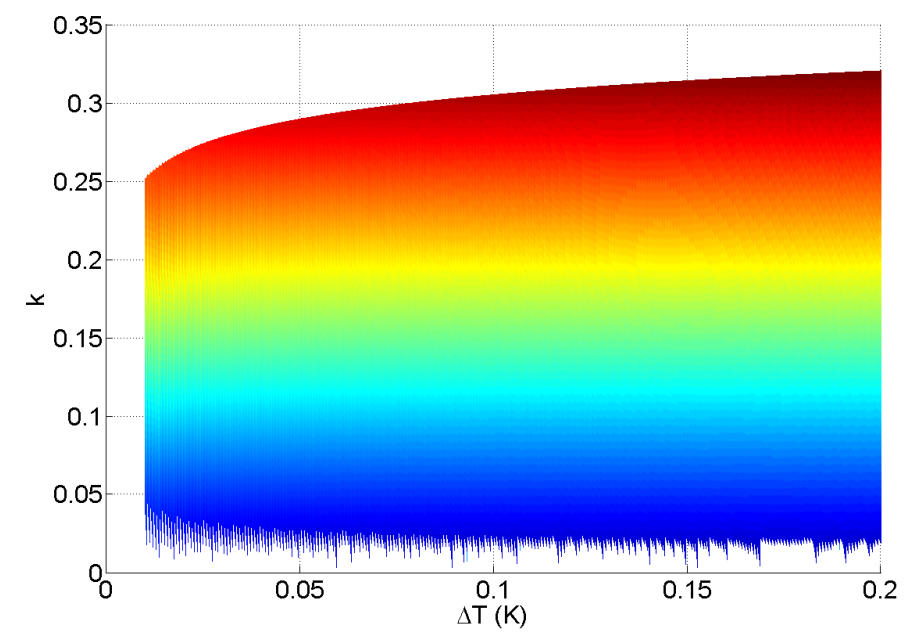

Figure 42: Cutoff wavenumbers based on $k$ and $\Delta T$.

As the superheat increases the range of cutoff wavenumbers increases, though 
at a rate that appears asymptotic. Though the full extent of the domain cannot be investigated, if the cutoff wavenumber is of an asymptotic nature, then there may potentially be a cutoff wavenumber. Such a case would then limit the range of disturbance wavenumbers capable of inciting instability. Thus, it is possible that for disturbance wavenumbers greater than the prescribed cutoff wavenumber, the film is always stable.

Looking at the perturbation growth rate, a sample wavenumber is selected so as to bisect the cutoff wavenumber surface. Figure 43 illustrates the perturbation growth rates dependence on the superheat for a perturbation wavenumber of 0.15 in a 100 um channel.

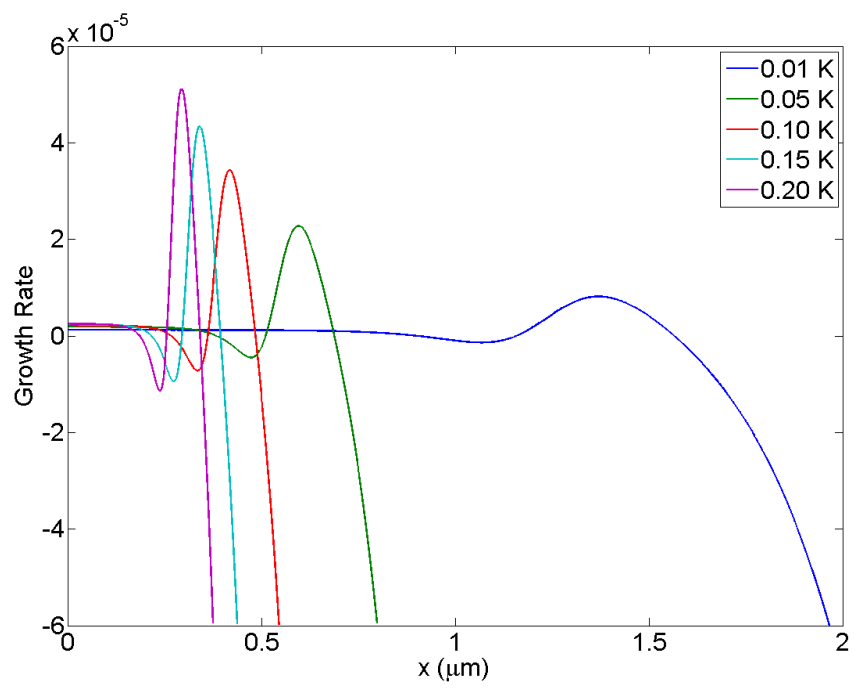

Figure 43: Perturbation growth rates for n-pentane and $k=0.15$.

The perturbation growth rates are noted to be of a smooth and continuous nature, unlike the sporadic solutions found for the MYL case. The perturbation growth rate begins positive nearest that non-evaporating thin-film region and transitions toward stability. The initial instability region is consistent with the findings of the flat and MYL cases presented previously.

As the solution shifts spatially, the stability region gives way to instability again. 
The second instability region is noted to have a maximum, with the peak value increasing with increasing superheat. The maximum growth rate then subsides giving way to unconditional stability toward the intrinsic meniscus region. Once again, the stability findings for the far field asymptotic boundary condition are consistent with those of the MYL case.

Given the spatial dependency of the thin-film properties and associated stability regions it may be possible to correlate instability with a specific thin-film property. The main contributors affecting thin-films are thought to be mass flux (vapour recoil), interfacial temperature gradient (Marangoni stress), liquid pressure gradient and the local interfacial curvature (capillary pressure).

The peak locations of each thin-film property can be compared to the peak growth rate location. As the thin-film length changes with superheat it is essential to normalise the results. Thus, the peak thin-film property locations are differenced with the peak growth rate location and normalised by the length of the thin-film as:

$$
D=\frac{x_{\text {peak }}-x_{\text {max }}}{\mathcal{L}}
$$

Taking the results of the perturbation growth rate and the thin-film properties, the final comparison is presented in Figure 44.

As can be seen from Figure 44, the peak locations of all of the suspected contributors asymptotically tend to the peak growth rate location within $1 \%$ or less. The mass flux and interfacial temperature gradients are nearly identical with the greatest degree of offset at the lowest superheats. Given the tendency of the properties to converge with increasing superheat, it is not possible to make a clear indication as to the principle contributor to the instability of the thin-film. 


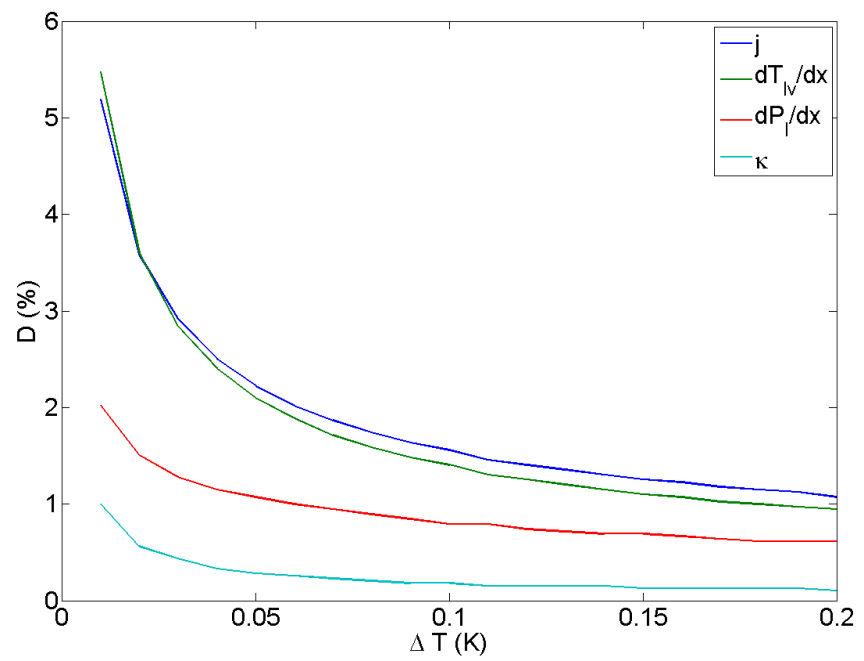

Figure 44: Comparison of the peak thin-film properties and perturbation growth rate locations.

\subsubsection{Summary}

A stability model describing an evaporating thin-film was developed. The model associated the potential for instability with an applied disturbance wavenumber and the geometry of the evaporating liquid film. Three different film geometries were considered: flat, circular (MYL) and curved. The stability of the flat film was dictated by the applied perturbation wavenumber and film thickness. The MYL base state (circular), introduced a spatial aspect to the stability. The early portion of the MYL case was found to be sporadically unstable and transitioned to a region of unconditional stability in the intrinsic region. Lastly, the evaporating thin-film model (curved) was asymptotically bounded by the flat film and Young-Laplace cases. The asymptotic bounds of the evaporating thin-film analysis were consistent with the flat and MYL cases studied previously. The intermediate region was found to have two distinct regions of instability and an intermediate region of stability. The location where the perturbation growth rate was largest was correlated with the convergence of 
the peak values for evaporation, curvature, liquid pressure gradient and surface temperature gradient. Therefore, it is possible to conclude that an evaporating meniscus can destabilise with the appropriate disturbance. Furthermore, the location of the potential rupture is expected to occur in the thin-film region. 


\section{Chapter 6}

\section{Meniscus instability - experiments}

The potential for evaporating liquid films to destabilise has been extensively studied from a theoretical perspective as outlined in Chapter 2 and Chapter 5. To advance the study of meniscus instability an experimental investigation is needed. Such experimental studies of evaporating meniscus instability are few in number [94,103,112] and are not consistent in geometry or environment. Thus, a set of experiments were to be designed, constructed and tested to validate the theoretical instability model.

\subsection{Experimental setup}

The experimental design required comparability between the linear stability model and the experiments. To achieve this, the assumptions regarding geometry, fluids and the environment were considered. Thus, the experiments were to be performed in high-aspect ratio channels contained within a vacuum chamber as shown in Figure 45. The vacuum chamber was placed on a large granite table and levelled using the sand bed. The entire setup was then placed on a set of airbags and rubber footings. The airbags and the rubber footings limited the transmission of external perturbations to the vacuum chamber setup to avoid any un-intentional triggers for the instability. 


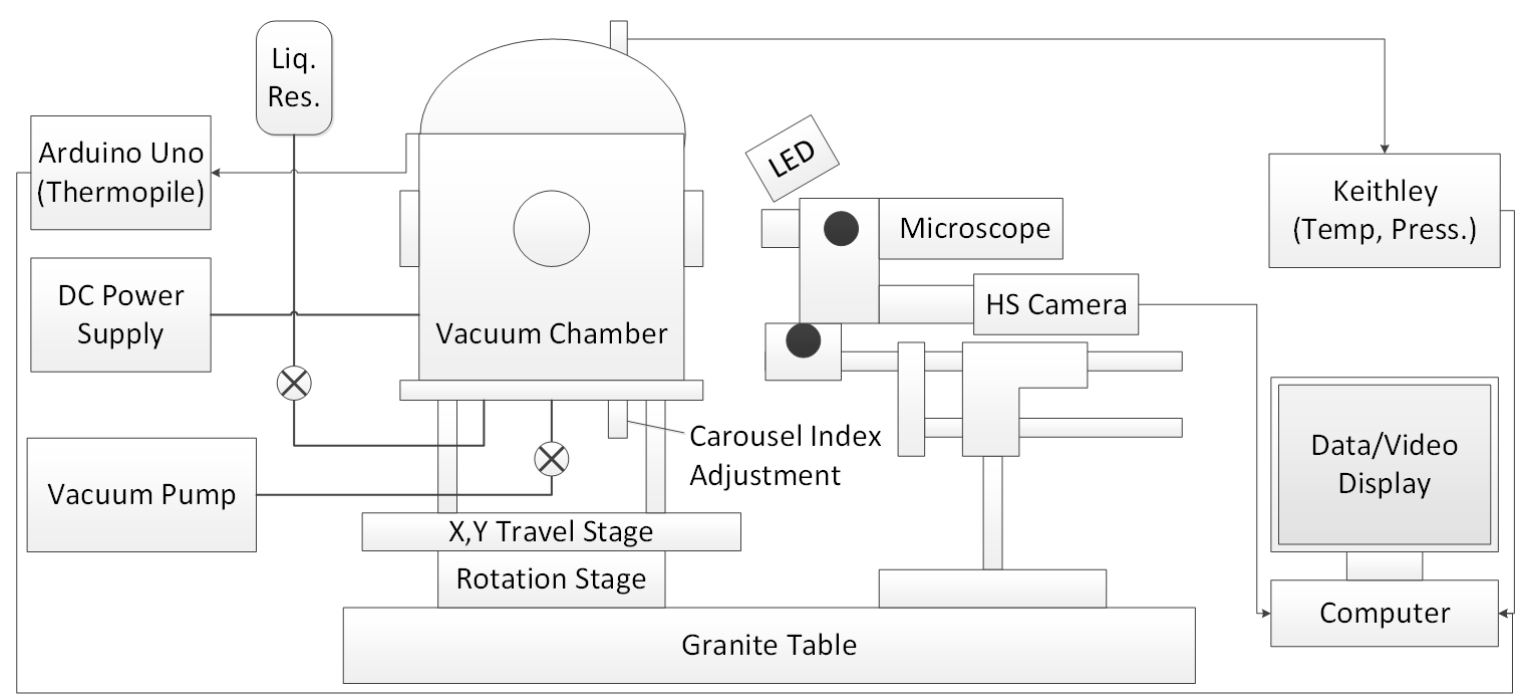

Figure 45: Experimental setup on the granite table.

The use of the vacuum chamber allowed for the evacuation of air from the system, allowing for the chamber to be charged with a single working fluid resulting in a saturated single species environment. The vacuum chamber was sufficient in size to approximate the vapour field as uniform. The top of the vacuum chamber was fitted with a pressure transducer (Omega PX429-015A5V) with an accuracy of $\pm 81 \mathrm{~Pa}$.

The vacuum chamber was fitted with two different types of thermal instruments. The first was a pair of T-type thermocouples (Omega 5SRTC-GC-T-30-36) with an accuracy of $\pm 0.5 \mathrm{~K}$. One thermocouple was placed in the liquid supply reservoir and the second placed in the ambient vapour field. The purpose of the thermocouples was to provide the liquid and vapour field temperatures so that the fluid properties could be obtained. The second thermal instrument was a thermopile array (Melexis 90620) installed in a bridge over the liquid reservoir aimed toward the capillary channel heater as shown in Figure 46. The thermopile array provided a non-invasive method of measuring the channel wall temperature distribution in-situ. The thermopile array had a field of $4 \times 16$ pixels with the 16 oriented in the vertical direction. Each pixel has an equivalent temperature detection of $0.08 \mathrm{~K}$ and a central pixel accuracy of \pm 1 


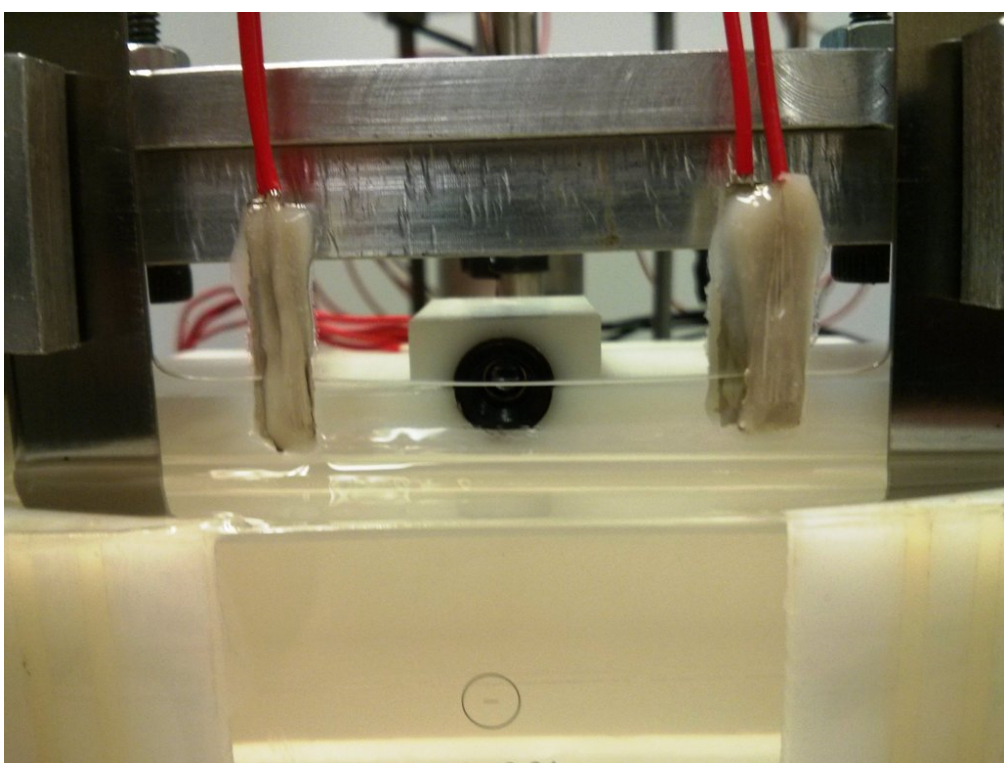

Figure 46: Thermopile installation.

$\mathrm{K} \pm 0.015\left|T_{\text {target }}-T_{\text {amb }}\right|$.

Inside the vacuum chamber a three experiment carousel was installed as shown in Figure 47. The setup was designed to hold three different capillary channels and provide a rotational freedom such that each experiment could be indexed over the liquid reservoir and permit observation through the view port on the vacuum chamber. This design permits the testing of three channels in succession without the need to re-draw vacuum and recharge the system with working fluid.

Inside the vacuum chamber the liquid reservoir was installed on an automated lift system to permit the raising and lowering of the reservoir so that the indexed experimental channel may be filled and supplied with liquid. The reservoir was positioned in front of the view port and below the experimental index point. The front portion of the reservoir was machined out and fitted with a transparent stage micrometre. The stage micrometre allowed for calibration of the microscope reticle in-situ while permitting observation of the reservoir interface. The bridge holding the thermopile 


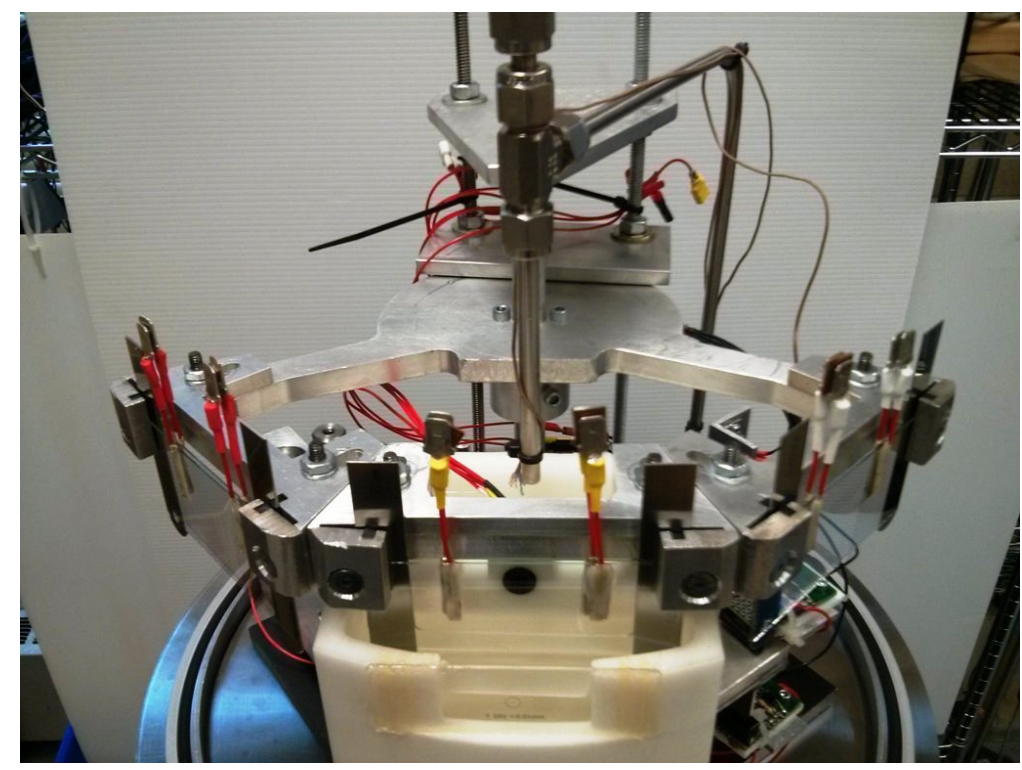

Figure 47: Three experiment carousel.

array was attached on the rear section of the reservoir and aimed at the channel from the rearward side thus allowing unobstructed viewing of the meniscus in the channel.

The capillary channels were constructed from a pair of glass plates dimensioned: $38.1 \times 82.6 \times 0.5 \mathrm{~mm}$. The glass plates offered an unobstructed view of the meniscus. To achieve the required spacing of the glass plates, a pair of Mitutoyu stainless steel feeler gauges (184-303S) were installed on the outboard edges of the glass plates. The spacers reduced the channel width by $25.4 \mathrm{~mm}$. The channel widths ranged from 0.3-1.0 $\mathrm{mm}$ in width, thus giving an aspect ratio range of 45-190. This large aspect ratio marginalised the end effects and ensured the central portion of the meniscus was essentially two dimensional to maintain consistency with the linear stability model. The glass plates and spacers were then mechanically clamped in a jig, thereby sealing the channel ends and forming a single complete capillary channel. As per the carousel, three sets of jigs holding different channel widths could be assembled and prepared for testing.

The channel walls were heated using a transparent conductive coating of Indium Tin Oxide (ITO). The glass plates were coated with a layer of ITO with a nominal 


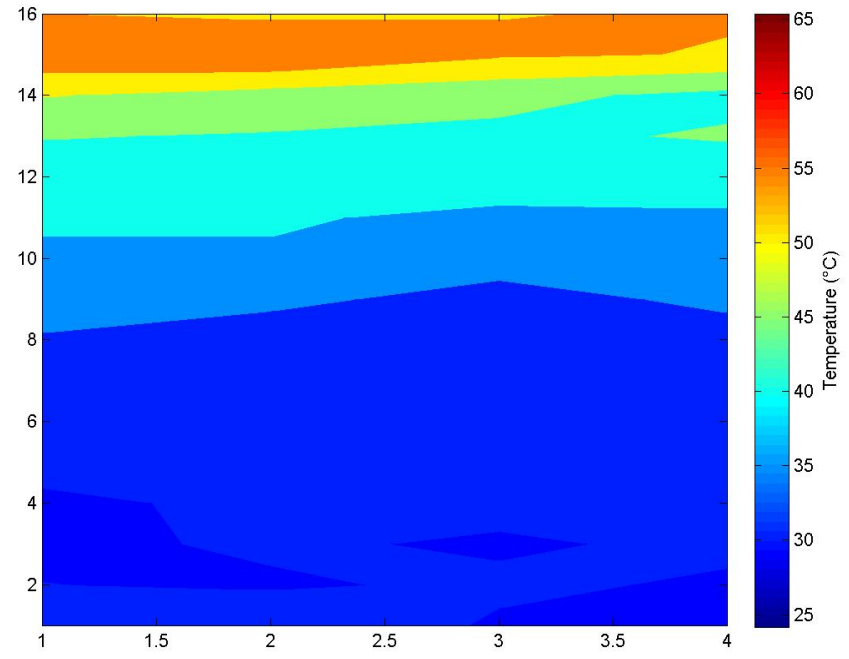

Figure 48: Typical temperature channel distribution as seen from the thermopile array.

resistance of $10 \Omega /$ Square area and patterned to achieve a patch heater located on the top half of the channel. The patch was centred laterally with dimensions: $19 \times 38.1$ mm. To power the heater a pair of silver epoxy (EPO TEK H20E) bus bars were applied to the outer portions of the ITO heater and affixed with electrical leads. The leads and bus bars were then coated with a layer of epoxy (Devcon HP 250) to provide mechanical strength and chemical protection. Once completed the glass plates were paired up and the heater tested for uniformity. An example of the heat distribution on the glass surface with a meniscus in the channel is shown in Figure 48.

Outside of the vacuum chamber, the thermocouple, pressure and heater voltage data were collected using a Keithley 2700 DAQ 22 bit analogue-to-digital converter with a conversion accuracy of $\pm 0.8 \%$ at a sampling rate of $1 \mathrm{~Hz}$. The thermopile array was run using an Arduino Uno with a sampling rate of $1 \mathrm{~Hz}$. The data from the thermopile array was recorded and saved for post processing.

Observation of the meniscus and recording of the instability was performed by way 
of the vacuum chamber view port. An optical stereo-microscope (Olympus SZH-10) was fitted with a reticle in the eyepiece. For recording the meniscus dynamics a high speed camera (Motion scope M1) with a lens (Fujinon CF25HA-1 1:1.4/25 mm) was installed on the auxiliary viewer.

\subsection{Experimental procedure}

The experiments were performed in a manner designed to ensure repeatability and consistency in the results. To meet this objective the vacuum chamber and all internal components were decontaminated using acetone, heated to evaporate any residues and then blown down with dry nitrogen. The whole system was then assembled in the chamber and instrumented. The test channels were then cleaned with acetone and assembled into the mechanical clamping jigs. The three experimental channels were then installed on the carousel.

The vacuum chamber was then closed and all ports sealed and prepared for drawing vacuum. A roughing pump (Alcatel Pascal 2000) was then used by way of a line valve to draw a vacuum of at least 50 mTorr. The chamber vacuum was then isolated by way of a line valve. A external fluid supply reservoir was then filled with working fluid and then the supply line was opened to the vacuum chamber. The chamber then began to fill with working fluid vapour until such a point that saturation pressure had been reached. Then the internal liquid supply reservoir was filled with working fluid. The charging process resulted in a lower chamber temperature than that of the laboratory, so a period of time was permitted for the system to reach thermal equilibrium with the laboratory. The chamber was considered to have reached equilibrium when the liquid and vapour temperatures were within $1{ }^{\circ} \mathrm{C}$ of the laboratory temperature.

The first experiment was then indexed and the internal reservoir raised thereby filling the capillary channel. The microscope eyepiece reticle was then calibrated 
using the stage micrometre on the front of the liquid supply reservoir. The meniscus height was then set using the liquid supply reservoir such that the meniscus was positioned within the ITO patch heater. The meniscus was then observed via the stereo-microscope and an initial height measurement taken. The height measurement was taken from the bottom of the meniscus to the free surface in the liquid supply reservoir. This height measurement provided a baseline as to the thermal equilibrium state of the meniscus at the start of the experiment.

As noted in Chapter 3, changes in the evaporation rate and fluid properties will lead to a change in meniscus height. Thus, as the meniscus is heated its height will change in response to the changes in the evaporation rate and surface tension will decrease. Given the meniscus will move with the application of heat, the heat should be applied in small increments with a period of time between each increment to allow the meniscus to transition to its new equilibrium position.

The heating sequence used a step-wise voltage profile with each step being held for one minute after the meniscus has achieved its new equilibrium position. A DC power supply (GPS-1830D) was used to supply the specified voltage and the current as necessary. Upon starting the experiment the instrumentation was initialised and data collection stored on a computer. The heater voltage profile was then started with the meniscus under constant observation. Upon the onset of instability, the meniscus height was measured in the same method as outline previously. The instability was observed for a period of one minute to ensure the instability was sustained.

With the instability confirmed to be sustained the power supply voltage was reduced and the meniscus permitted to re-stabilise. The stepwise heater process was then started and the meniscus instability triggered. This was repeated until five data points were collected. On the fifth data collection set, the high speed camera was used to capture the motion of the meniscus for further analysis. Upon collection of 
the pertinent data, the heater voltage was increased an additional two volts to determine if the instability persisted for higher superheats. Upon completion of a channel experiment the reservoir was lowered and the next channel indexed and the reservoir was topped off with working fluid.

This procedure was repeated for each experiment in the carousel. When all three test channel experiments had been completed the setup was permitted to cool down prior to opening the chamber to the environment to reduce the risk of fire. Once cooled, the vacuum chamber was opened to the ambient. The whole assembly was dismantled in preparation for cleaning. Once again the setup was cleaned with acetone and heated to remove trace acetone and then blown down with dry nitrogen and then re-assembled. This entire procedure was completed between each experimental run and for each working fluid.

\subsection{Superheat measurement options}

Many theoretical works rely on an assumption that the superheat, or temperature jump across the interface is known. Experimentally however, this measurement is incredibly difficult to obtain. Earlier methods have used thermocouples [35] near the surface or in some cases along the side of the capillary wall [109,112]. Others have used infrared imaging techniques to map out the wall temperature profiles. Each technique has its merits; each also has detractions, as with any other method.

Measurements with a pair of thermocouples across the interface are exceptionally difficult as the interface is contained within a sub-millimetre sized channel. Further complicating matters is the use of a vacuum chamber and the limited access to the system. Lastly, as the instability is assumed to occur in the evaporating thin-film region, the scales of the problem as compared to the instruments and temporal response rates make such measurements nearly impossible. Such complexities inevitably lead 
to the notion that if such measurements were to be collected, their accuracy and repeatability may come into question.

As for the techniques of placing thermocouples on the side of the channel wall, the changes in meniscus height must still be addressed. Though the technique is may be simpler to implement, the temperature distribution in the glass wall is offset from that actually found across the liquid-vapour interface. As with the previous thermocouple method, the scales of the problem make such measurements questionable at best. Furthermore, the use of two thermocouples in this fashion is reliant upon the assumption that the temperature profile in the glass nearest the thin film is of a linear profile.

Lastly, the non-invasive method of infrared measurement can be employed. Studies using this method [9] have been able to capture the temperature profile of the wall near the meniscus. The results of such work have demonstrated the application of thermocouples on the wall to be invalid given the non-linear nature of the temperature near the top of the meniscus. Despite the marked advantages of the infrared system, its application to this problem is not readily available. The principal problem with this method is the need of access to the channel wall. Given that the channel wall is inside the vacuum chamber, it would require the replacement of the glass view port with an infrared transparent glass that is vacuum rated.

Given the difficulties with each technique, an alternate method can be used. As outlined in Chapter 3, a model was developed to describe the motion of a meniscus as it filled the capillary tube while evaporating. The model and its validation had been performed with some degree of success. While the capture of the meniscus dynamics remains in need of refinement, this application is limited to a steady state problem. Should the dynamic aspect of the imbibition model be removed, it can be used to correlate a height measurement with a theoretical superheat. 
This method allows for the inference of the microscopic superheat across the interface from a simple height measurement. While Pratt et al. [109,118] recorded such values in their experimental work, there was no means of relating it to something tangible. With the development of the imbibition model, it is now possible to relate the meniscus height measurement to that of a microscopic superheat.

\subsection{Superheat model}

As the development of the imbibition model has been previously outlined for the case of a cylindrical capillary tube, the following will briefly outline the modification of the model to match the channel configuration. Starting once again from the equation of motion for an evaporating meniscus for a channel:

$$
\rho \frac{d}{d t}(u h)=\frac{2 \sigma}{W}-\rho g h-\frac{12 \mu h u}{W^{2}}-\left(\frac{\rho-\rho_{v}}{\rho \rho_{v}}\right) j^{2}
$$

As before, the equation assumes the interface to be of an average height $h$ as though it were a flat interface. This height is measured from the free interface of the reservoir to the meniscus. As the meniscus is evaporating, a replenishing supply of liquid from the supply reservoir is drawn up into the channel. This motion must overcome a viscous pressure drop. While the same is applicable to the vapour side, the scales of the experimental setup coupled with the small viscosity lead to the option of neglecting the vapour pressure drop. The rate at which the fluid is replaced can be determined by applying continuity across the interface and finding the average fluid velocity as:

$$
u=\frac{j}{\rho}
$$

where $j$ is the evaporative mass flux which can be described from the Clapeyron 
model as:

$$
j=a \Delta T
$$

Lastly, the experimentally measured height must be related to the average meniscus height. Using an area average technique, the average height of the meniscus is related to the experimentally measured meniscus height by:

$$
h=h_{\exp }+\frac{W(4-\pi)}{8}
$$

Substitution Equations (144) - (146) into Equation (143) and apply the steady state condition we get:

$a^{2}\left(\frac{\rho-\rho_{v}}{\rho \rho_{v}}\right) \Delta T^{2}+\frac{12 \mu a}{W^{2} \rho}\left(h_{\text {exp }}+\frac{W(4-\pi)}{8}\right) \Delta T+\rho g\left(h_{\exp }+\frac{W(4-\pi)}{8}\right)-\frac{2 \sigma}{W}=0$

The result is that of a quadratic equation in superheat as a function of the experimentally measured meniscus height and the fluid properties. This model allows for the correlation of a non-invasive meniscus height measurement to a microscopic superheat value consistent with the evaporation model used previously.

\subsection{Experimental results}

A set of experimental tests were performed for three different fluids (n-pentane, isooctane and acetone) for channel widths ranging from $0.3-1.0 \mathrm{~mm}$. Following the previously outlined experimental procedure, the channel based meniscus was heated using the ITO patch heater. Following the stepwise heater voltage profile, the meniscus was observed for signs of instability. Upon the onset of instability the meniscus height was collected. After five repeated runs, the heater voltage was increased incrementally an additional two volts to ensure the instability was sustained for higher 
heating loads.

\subsubsection{Instability nature}

For the two alkanes, n-pentane and iso-octane, the meniscus was observed to destabilise. The instability was consistently repeated, however the increase in heater voltage led to the re-stabilisation of the meniscus. The localised instability was only observed for the alkanes. Using data collected from the high speed camera, the specifics of the motion were captured. Using a frame rate of 250 frames per second (fps) the data was collected and post processed in MATLAB. A single column of pixels from each frame was extracted and sequenced to form a type of particle motion plot. After some manipulation, the motion plots are obtained, with a pair of typical plots shown in Figure 49.

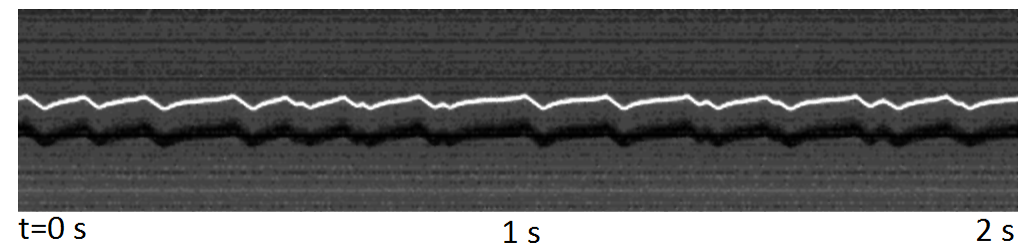

(a) n-pentane.

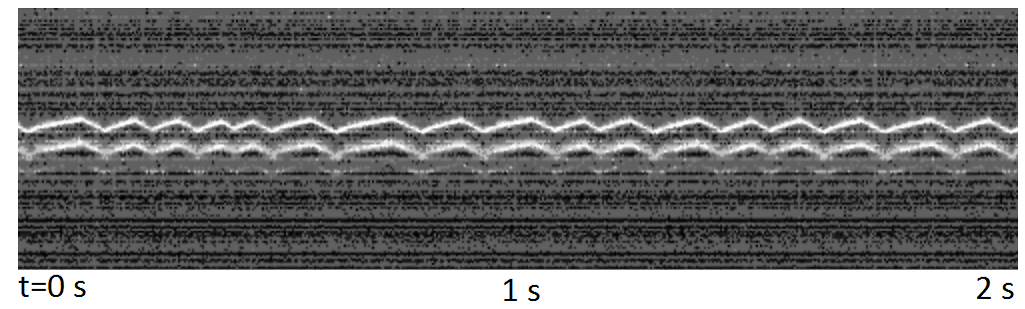

(b) iso-octane.

Figure 49: Typical instability motion profiles for low superheat instability.

The motion of the meniscus as illustrated in Figure 49, spans a period of two seconds with a consistent oscillation amplitude thereby confirming the oscillation is sustained. The oscillation appears to be of a small amplitude and high frequency 
with a triangular wave form. For the n-pentane case, the meniscus rises gently for a period of time before falling at a rate greater than that at which it rose. This general observation also holds for the iso-octane case.

The motion data was enhanced with MATLAB's built-in edge finding algorithms and the results filtered to remove noise. The resulting enhanced and filtered data was then analysed using a Fast Fourier Transform (FFT). The FFT results corresponding to the typical cases are depicted in Figure 49 are presented in Figure 50.

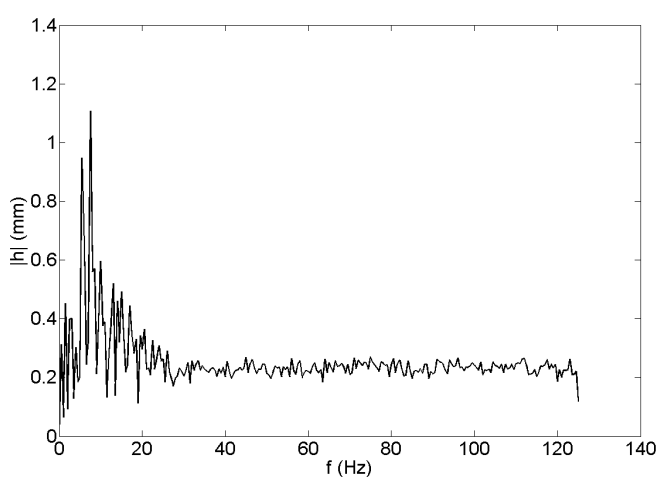

(a) n-pentane.

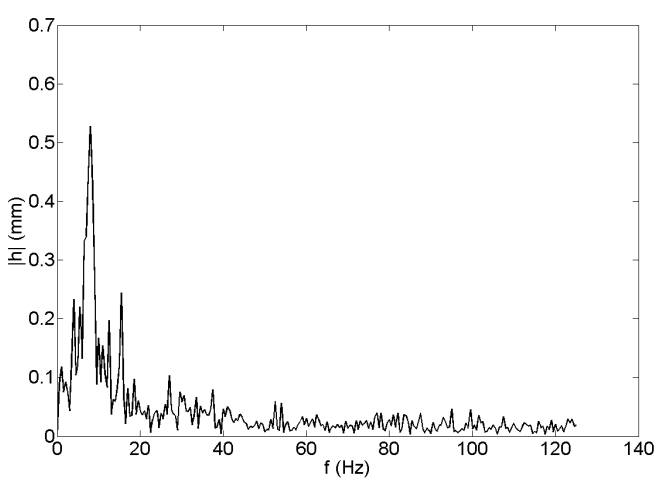

(b) iso-octane.

Figure 50: FFT data for the typical instability motion profiles.

The FFT analysis of the example cases of n-pentane and iso-octane revealed peak frequencies at $7.5 \mathrm{~Hz}$ and $6.5 \mathrm{~Hz}$ respectively. An extended evaluation of the data collected for the range of channel widths did not yield any viable correlations between the oscillation frequency and the channel width. Thus, the contributing factors affecting the oscillation profile appear to be unrelated to the channel width.

When the heater voltage was increased, the instability for n-pentane and isooctane subsided. The heater voltage was then increased as before until a sustained oscillation was found to occur. The meniscus instability found for the higher superheats was found to occur for all three fluids tested: n-pentane, iso-octane and acetone. 


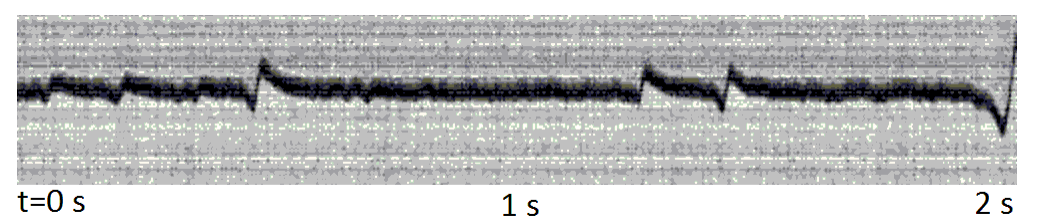

(a) n-pentane.

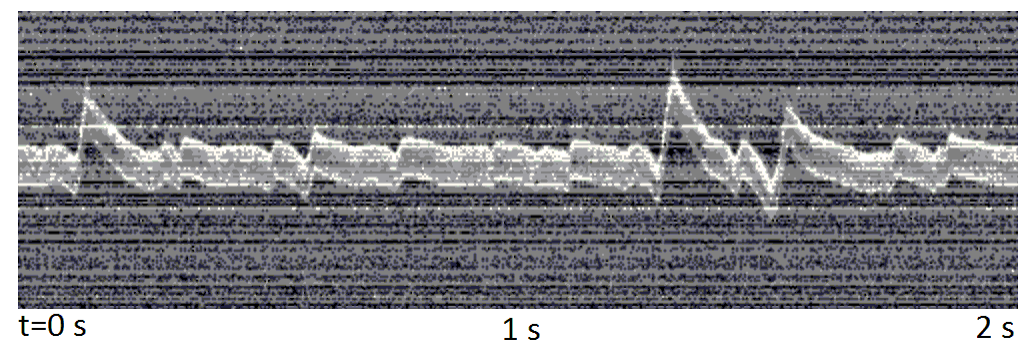

(b) iso-octane.

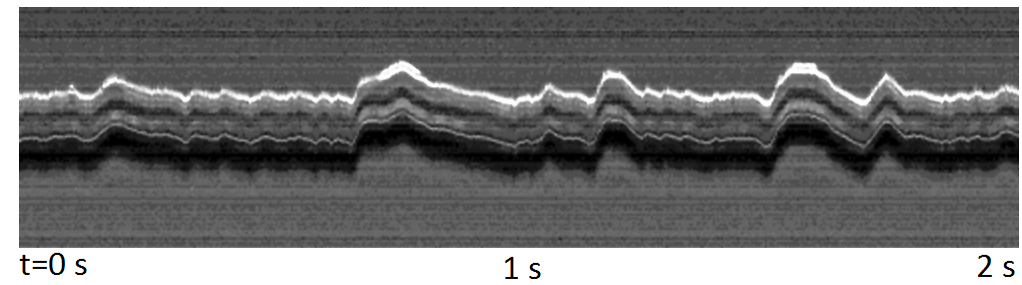

(c) acetone.

Figure 51: Typical instability motion profiles.

As before the height data was collected for five instability runs per channel and high speed video collected. Again the heater voltage was increased an additional two volts to determine the sustainability of the oscillation for higher heater setting. For all three fluids tested the oscillation was found to remain for the additional two volt increase in heater setting. As a test, an instability test was performed with the heater voltage increased 10 volts beyond the onset of the second kind of instability. The instability was observed to be sustained for all higher heater settings, with the interface continuing to recede in height as before.

The nature of the second kind of instability was captured by the high speed camera at a rate of $250 \mathrm{fps}$. The frames were analysed as before with typical motion plots provided in Figure 51. 
The motion of the unstable meniscus for the second kind of instability is notably different from that of the first kind observed for the alkanes. The second kind of instability appears to be comprised of random small- and large-scale oscillations. The data was again analysed using an FFT with no discernible correlations between the peak frequencies and the channel width.

The motions for n-pentane and iso-octane were similar with the small and large scale motions having a sharp rise to peak and then a drawn out descent. In the case of acetone, the small and large scale motions are of a smoother nature as compared to the alkanes. For acetone, the rise and fall rates are more distributed in time. For

all three fluids, the small scale oscillations appear to be consistent throughout the interval of time studied while the large scale motions are more sporadic. The large scale motions appear to be preceded by a drop in meniscus height. The preceding drop in height is slightly larger than the amplitude of the small scale motions. It can then be deduced that a perturbation of sufficient magnitude is required to trigger the large scale motion.

This experimental study is the first to identify the existence of two different kinds of instability in an evaporating meniscus. The first kind of instability appears to be unique to n-pentane and iso-octane and it is speculated that other alkanes may share this instability mode. The second kind of instability was found to occur for all of the fluids tested independent of their composition or polarity. Furthermore, the second kind of instability was found to be sustained for a larger range of heat values. Thus, the second kind of instability is our principal concern for applications utilising evaporating menisci.

\subsubsection{Instability onset}

During the experiments, the height of the meniscus was recorded so that it may be correlated with a superheat value. As the sustainability of the second kind of 
instability is of principal concern, the height values for the second instability are presented for a range of channel widths in Figure 52.

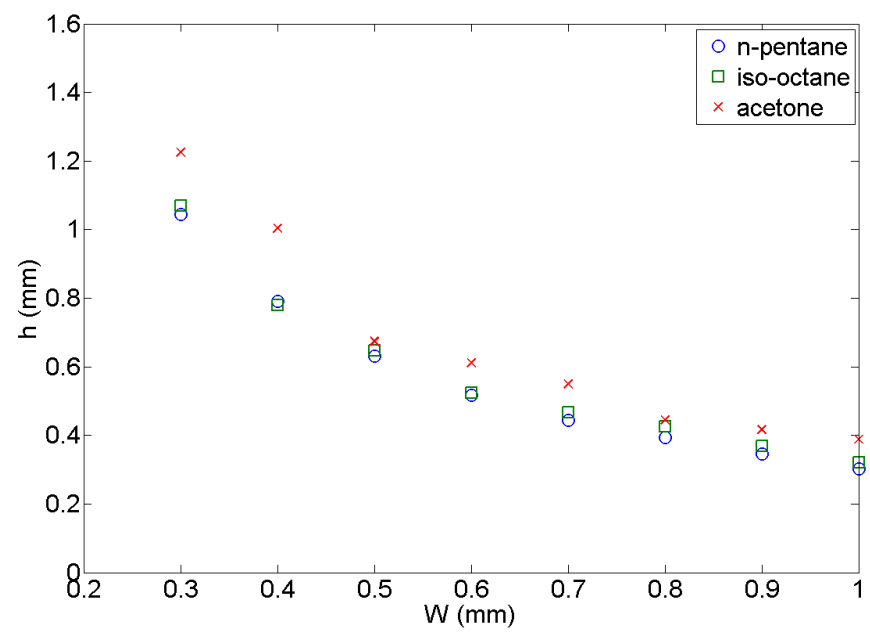

Figure 52: Meniscus height at the onset of the second kind of instability for npentane, iso-octane and acetone.

As expected, the height is found to decrease with increasing channel width as is consistent with the Young-Laplace formulation. The destabilisation heights of the n-pentane and iso-octane are nearly identical, with acetone following a similar trend with a slight offset. The errors attributed to the height measurement were $\pm 0.07 \mathrm{~mm}$ and are of a size comparable to the symbols used in the plot.

The measured meniscus heights can then be applied to Equation (147) to infer the superheat. Figure 53 illustrates how the superheat and fluid are related to the channel width. 


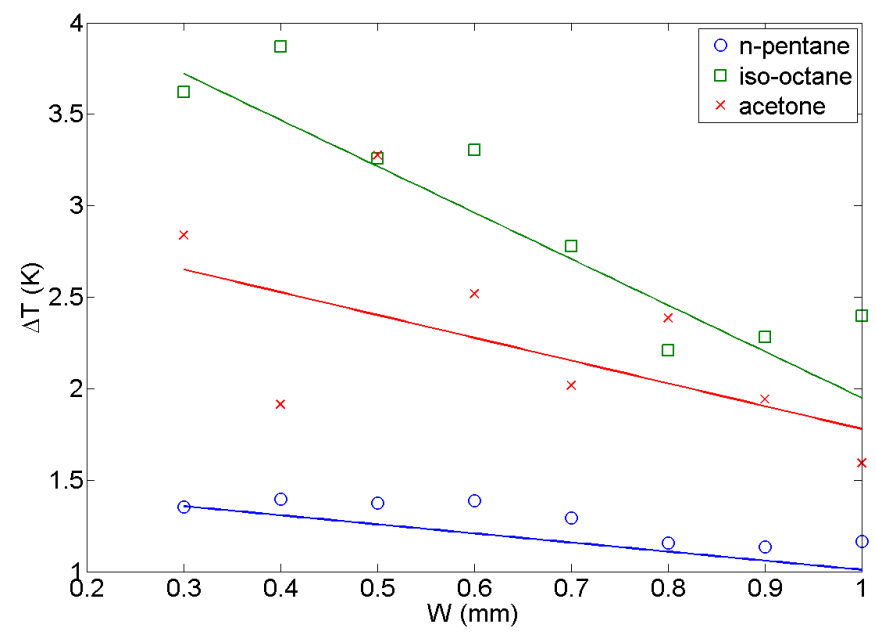

Figure 53: Unstable superheats for the second kind of instability for n-pentane, iso-octane and acetone.

While the meniscus heights at the onset of instability were in close proximity, the application of the superheat model redistributed the fluids based on superheat. The superheats required to destabilise a meniscus are all found to be less than $4 \mathrm{~K}$. The onset of instability appears to be a function of the channel width such that larger channels are destabilised by smaller superheats.

\subsection{Summary}

A series of evaporating channel based meniscus experiments were performed for three fluids and eight channel widths. When the heat was applied the meniscus height was observed to reduce in height until it achieved a new equilibrium height that is consistent with the meniscus dynamics model, thus further supporting the observation that temporal changes in heating load result in interface motion. Through the course of the experiments, two different instabilities were observed for n-pentane and isooctane, and only one was observed for acetone.

The experimental findings are the first of their kind to identify the existence of two 
different instabilities. The first instability was determined to be unique to n-pentane and iso-octane and was found to occur for a narrow range of heater settings. Increased heating beyond the first instability was found to re-stabilise the meniscus. This is the first indication that the first kind of instability is limited both in thermal requirement as well as fluid type. The second instability was found to occur for all three fluids tested and confirmed to be sustainable for higher superheats. For oscillations of the second kind, each fluid exhibited an increase in superheat with decreasing channel width. Despite similarities in the chemical nature and structure of n-pentane and iso-octane, the superheat required for instability was notably different in its relation to the channel width. 


\section{Chapter 7}

\section{Concluding remarks}

\subsection{Conclusions}

In this work, a mathematical model was developed to describe the dynamics of an evaporating meniscus. The model was able to capture how phase change at the interface could contribute to the statics and dynamics of an evaporating meniscus. Through this theoretical analysis, it was discovered that the superheat could be correlated with meniscus height. This fundamental finding provided a means by which to correlate a non-invasive height measurement with that of a theoretical superheat value.

The theoretical study of evaporating meniscus dynamics was succeeded by a series of evaporating meniscus experiments. The experiments were performed for three different working fluids, three capillary tube diameters and five heater settings. The experimental results were found to be consistent with the trends predicted by the meniscus dynamics model (imbibition model). The model was found to be accurate during the initial rise and final equilibrium portions of the dynamics, with varying degrees of success in capturing the interim dynamics. These findings are consistent with the assumptions made in the development of the meniscus dynamics model, as

these regions of the experiment were consistent with the unidirectional laminar flow 
assumption.

Among the meniscus dynamics experimental findings, the most important is the confirmation that evaporation (and any potential transients) can have a measurable effect on the meniscus dynamics and equilibrium height. Although the transient evaporation and meniscus dynamics require further refinement, the model is valid when the flow properties are primarily unidirectional and laminar. Therefore, the imbibition model can be applied during the initial rise phase and when the evaporating meniscus has achieved a stable equilibrium height. As the model is valid for these flow regimes, the experimental evidence hereby supports the hypothesis that the superheat can be correlated with a measurement of meniscus height.

The meniscus dynamics study was followed by the development of a linear stability model for an evaporating meniscus. The stability model associated the potential for interfacial instability with an applied disturbance wavenumber and the liquid film geometry. The geometric component originates with the liquid film geometry. In the case of an evaporating meniscus it was presumed that the interface is comprised of differing geometric regions.

An investigation of evaporating film stability was performed for the three different base cases. The stability of the flat film was found to be dictated by the applied perturbation wavenumber and film thickness. The perturbation growth rates were noted to increase with increasing superheat for positive growth rate values and wavenumbers lower than the cutoff wavenumber. Any spatial aspects of the stability were removed with the higher order derivatives all taking values of zero.

Implementation of the modified Young-Laplace (MYL) base state resulted in the introduction of spatial contributions. Similar to the flat film case, the MYL base state can be described by a closed form equation which is continuous and differentiable. The development of the stability model was limited in its application in accordance with the assumption: $\zeta H_{X} \ll 1$. As such, only a portion of the MYL solution could 
be studied. Of the viable section studied, the film was noted as being unconditionally stable in the intrinsic region of the meniscus. Nearing the thin-film region, the perturbation growth rate tends toward zero and then begins to sporadically jump between positive and negative perturbation growth rates. A three dimensional analysis of the perturbation growth rate showed that each jump in the perturbation growth rate was on a diverging trajectory with increasing wavenumber. While the film may be expected to be unstable as it approaches the flat film case, the sporadic nature of the perturbation growth rate near the flat region is unique.

The third case studied used an evaporating thin-film model previously developed as the base state. The evaporating thin-film model was expected to most accurately reflect the conditions of the transitioning region for an evaporating meniscus. The thin-film model was asymptotically bounded by the flat film and Young-Laplace conditions and was expected to have similar traits to the flat and MYL case studies. The results of the thin-film analysis were found to be consistent with the flat and MYL cases in the asymptotic ends.

The thin-film was found to have two distinct regions where instability might occur in addition to an intermediate region of unconditional stability. The first region of instability appeared to be a continuance from the non-evaporating thin-film region as is consistent with the flat film case analysed previously. As the instability region progressed along the film toward the bulk region, the instability diminished as it gave way to an unconditionally stable region. The stable region was spatially narrow, after which the second instability region began to develop. The second instability region had a maximum that shifted spatially with superheat suggesting a potential correlation between the thin-film solution and instability. Subsequent analysis of the thin-film properties and the peak growth rate locations indicated the peak instability was driven by a convergence of the thin-film properties and geometry. Furthermore, the spatial dependence was noted to shift the peak instability region toward the 
non-evaporating thin-film region with increasing superheat. The thin-film analysis presented in this work is the first mathematical evidence implicating the thin-film region as the likely the source of instability.

The theoretical study of meniscus instability was followed by a series of evaporating channel based meniscus experiments. The experiments were performed for three fluids and eight channel widths. The experiments used thin glass sections for the channel walls and a patterned ITO for the heater to allow for direct observation of the meniscus. The ITO provided a near uniform heat distribution on the test section, thereby ensuring the test section could be considered two dimensional. The resulting channel meniscus was found to be uniform with end effects beyond the heated test section. Thus, the meniscus test section was consistent with the mathematical assumptions of the meniscus stability analysis.

When the heat was applied, the meniscus was observed to reduce in height until it achieved a new equilibrium as is consistent with the meniscus dynamics model and experimental findings. Through the course of the heating profile, two different instabilities were observed for n-pentane and iso-octane, while only one was observed for acetone. The finding of two different kinds of instabilities is a first in current record. The first instabilities observed for n-pentane and iso-octane were at lower power settings and found to occur for a narrow region of superheat. The early instability for n-pentane and iso-octane was noted to occur for a narrow range of heater settings and would re-stabilise with subsequent increases in heater power.

The second kind of instability was found to be sustained for all higher superheats and was the intended focus of the study. The meniscus height at which the instability occurred followed a natural progression downwards with increasing channel size. Using an appropriate form of the imbibition model, the superheats at which each fluid destabilised were obtained. The superheat necessary to destabilise the meniscus was 
found to decrease with increasing channel width. Despite similarities in the chemical nature of n-pentane and iso-octane, the superheat required for instability was different in its relation to the channel width.

\subsection{Future work}

The imbibition model was developed in a fashion such that it could capture the dynamics of a meniscus subject to phase change. While in its current formulation it can capture the interim dynamics for some of the studies, the non-linear evolution observed experimentally must be better resolved. This may be done by applying a prescribed transient thermal load to an initially static meniscus and observing the interface's response. Furthermore, the differences between non-polar (n-pentane) and polar fluids (water) must be addressed in future iterations. As a first step, the removal of the fully developed flow condition and the flat interface should be addressed.

While the focus of this study was on the potential for instability, the existence of a stable region nested between two unstable regions is of particular interest. The conditions governing this stable region should be further investigated to determine the principal contributors. Such information could potentially provide a means by which to delay the onset of interface instability.

The current stability model looks at the potential for instability based on the initial perturbation growth rate. As the evolution equation is available, a numerical method could be employed to investigate the interface evolution and confirm the hypothesis that the thin-film region is the source of the instability. Though the derivation includes curved surfaces, the evolution equation without the $\zeta H_{X} \ll 1$ assumption must be used to capture the dynamics closest to the point of rupture. If successful, the model's application could be expanded to other highly curved surfaces such as surface waves and droplets. 


\section{List of References}

[1] S. C. Maroo and J. N. Chung, "Heat transfer characteristics and pressure variation in a nanoscale evaporating meniscus," International Journal of Heat and Mass Transfer, vol. 53, pp. 3335-3345, 2010.

[2] M. Trainer, J. Stern, and P. Mahaffy, "Sample analysis at Mars: Updates." http://science.gsfc.nasa.gov/699/marsReferences.shtml, 2012.

[3] C. Höhmann and P. Stephan, "Microscale temperature measurement at an evaporating liquid meniscus," Experimental Thermal and Fluid Science, vol. 26, pp. $157-162,2002$.

[4] K. Park and K. S. Lee, "Flow and heat transfer characteristics of the evaporating extended meniscus in a micro-capillary channel," International Journal of Heat and Mass Transfer, vol. 46, pp. 4587-4594, 2003.

[5] M. Potash Jr. and P. C. Wayner Jr., "Evaporation from a two-dimensional extended meniscus," International Journal of Heat and Mass Transfer, vol. 15, pp. 1851-1863, 1972.

[6] S. DasGupta, I. Y. Kim, and P. C. Wayner Jr., "Use of the kelvin-clapeyron equation to model an evaporating curved microfilm," Journal of Heat Transfer, vol. 116, pp. 1007-1015, 1994.

[7] S. K. Wee, K. D. Kihm, and K. P. Hallinan, "Effects of the liquid polarity and the wall slip on the heat and mass transport characteristics of the micro-scale evaporating transition film," International Journal of Heat and Mass Transfer, vol. 48, pp. 265-278, 2005.

[8] B. Derjaguin, N. Churaev, and V. Muller, Surface forces. New York: Consultants Bureau, 1987. 
[9] K. Stephan, "Influence of dispersion forces on phase equilibria between thin liquid films and their vapour," International Journal of Heat and Mass Transfer, vol. 45, pp. 4715-4725, 2002.

[10] J. J. Zhao, Y. Y. Duan, X. D. Wang, and B. X. Wang, "Effects of superheat and temperature-dependent thermophysical properties on evaporating thin liquid films in microchannels," International Journal of Heat and Mass Transfer, vol. 54, pp. 1259-1267, 2011.

[11] P. C. Wayner Jr., "Mechanical and thermal effects in the forced spreading of a liquid film with a finite contact angle," Colloids and Surfaces A: Physicochemical and Engineering Aspects, vol. 89, pp. 89-95, 1994.

[12] W. Qu, T. Ma, J. Miao, and J. Wang, "Effects of radius and heat transfer on the profile of evaporating thin liquid film and meniscus in capillary tubes," International Journal of Heat and Mass Transfer, vol. 45, pp. 1879-1887, 2002.

[13] H. Wang, S. V. Garimella, and J. Y. Murthy, "An analytical solution for the total heat transfer in the thin-film region of an evaporating meniscus," International Journal of Heat and Mass Transfer, vol. 51, pp. 6317-6322, 2008.

[14] P. C. Stephan and C. A. Busse, "Analysis of the heat transfer coefficient of grooved heat pipe evaporator walls," International Journal of Heat and Mass Transfer, vol. 35, pp. 383-391, 1992.

[15] H. Wang, S. V. Garimella, and J. Y. Murthy, "Characteristics of an evaporating thin film in a microchannel," International Journal of Heat and Mass Transfer, vol. 50, pp. $3933-3942,2007$.

[16] J. B. Freund, "The atomic detail of an evaporating meniscus," Physics of Fluids, vol. 17, pp. 022104-1-9, 2005.

[17] K. Park, K. J. Noh, and K. S. Lee, "Transport phenomena in the thin-film region of a micro-channel," International Journal of Heat and Mass Transfer, vol. 46, pp. 2381-2388, 2003.

[18] P. C. Wayner Jr., Y. Kao, and L. LaCroix, "The interline heat-transfer coefficient of an evaporating wetting film," International Journal of Heat and Mass Transfer, vol. 19, pp. 487-492, 1976.

[19] J. A. Schonberg, S. DasGupta, and P. C. Wayner Jr., "An augmented YoungLaplace model of an evaporating meniscus in a microchannel with high heat flux," Experimental Thermal and Fluid Science, vol. 10, pp. 163-170, 2011. 
[20] H. Wang, J. Y. Murthy, and S. V. Garimella, "Transport from a volatile meniscus inside an open microtube," International Journal of Heat and Mass Transfer, vol. 51, pp. 3007-3017, 2008.

[21] S.-Y. Du and Y.-H. Zhao, "New boundary conditions for the evaporating thinfilm model in a rectangular micro channel," International Journal of Heat and Mass Transfer, vol. 54, pp. 3694-3701, 2011.

[22] Y. Zhang and G. Lu, "Flow factor for molecularly thin fluid films in onedimensional flow due to fluid discontinuity," Journal of Molecular Liquids, vol. 116, pp. 43-50, 2005.

[23] G. Ball, "Numerical analysis of the heat transfer characteristics within an evaporating meniscus," Master's thesis, Carleton University, 2012.

[24] S. S. Panchamgam, A. Chatterjee, J. L. Plawsky, and P. C. Wayner Jr., "Comprehensive experimental and theoretical study of fluid flow and heat transfer in a microscopic evaporating meniscus in a miniature heat exchanger," International Journal of Heat and Mass Transfer, vol. 51, pp. 5368-5379, 2008.

[25] C. Sodtke, J. Kern, N. Schweizer, and P. Stephan, "High resolution measurements of wall temperature distribution underneath a single vapour bubble under low gravity conditions," International Journal of Heat and Mass Transfer, vol. 49, pp. 1100-1106, 2006.

[26] Z. H. Kou and M. L. Bai, "Effects of wall slip and temperature jump on heat and mass transfer characteristics of an evaporating thin film," International Journal of Heat and Mass Transfer, vol. 38, pp. 874-878, 2011.

[27] C. Buffone, K. Sefiane, and J. R. E. Christy, "Experimental investigation of the hydrodynamics and stability of an evaporating wetting film placed in a temperature gradient," Applied Thermal Engineering, vol. 24, pp. 1157-1170, 2004.

[28] R. Ranjan, J. Y. Murthy, and S. V. Garimella, "A microscale model for thinfilm evaporating in capillary wick structures," International Journal of Heat and Mass Transfer, vol. 54, pp. 169-179, 2011.

[29] J. L. Polansky, "Numerical model of an evaporating thin film," Master's thesis, Carleton University, 2011. 
[30] S. S. Panchamgam, J. L. Plawsky, and P. C. Wayner Jr., "Microscale heat transfer in an evaporating moving extended meniscus," Experimental Thermal and Fluid Science, vol. 30, pp. 745-754, 2006.

[31] H. J. Butt and M. Kappl, Surface and Interfacial Forces. Federal Republic of Germany: Wiley-VCH Verlag GmbH and Co. KGaA, Weinheim, 2010.

[32] S. DasGupta, J. A. Schonberg, I. Y. Kim, and P. C. Wayner Jr., "Use of the augmented Young-Laplace equation to model equilibrium and evaporating extended meniscus," Journal of Colloid and Interface Science, vol. 157, pp. 332342, 1993.

[33] G. Ball, J. Polansky, and T. Kaya, "Investigation of particular features of the numerical solution of an evaporating thin film in a channel," Frontiers in Heat and Mass Transfer, vol. 4, pp. 1-9, 2013.

[34] M. Sujanani and P. C. Wayner Jr., "Microcomputer-enhanced optical investigation of transport processes with phase change in near-equilibrium thin liquid films," Journal of Colloid and Interface Science, vol. 143, pp. 472-488, 1991.

[35] G. Fang and C. Ward, "Temperature measured close to the interface of an evaporating liquid," Physical Review E, vol. 59, pp. 417-428, 1999.

[36] C. Ward and G. Fang, "Expression for predicting liquid evaporation flux: Statistical rate theory approach," Physical Review E, vol. 59, pp. 429-440, 1999.

[37] G. Fang and C. Ward, "Examination of the statistical rate theory expression for liquid evaporation rates," Physical Review E, vol. 59, pp. 441-453, 1999.

[38] R. W. Schrage, A theoretical study of interphase mass transfer. PhD thesis, Columbia University, 1953.

[39] P. C. Wayner Jr., "The effect of interfacial mass transport on flow in thin liquid films," Colloids and Surfaces, vol. 52, pp. 71-84, 1991.

[40] P. C. Wayner Jr., "Intermolecular forces in phase-change heat transfer: 1998 kern award review," AIChE Journal, vol. 45, pp. 2055-2068, 1999.

[41] S.-Y. Du and Y.-H. Zhao, "Numerical study of conjugated heat transfer in evaporating thin-films near the contact line," International Journal of Heat and Mass Transfer, vol. 55, pp. 61-68, 2012. 
[42] J.-J. Zhao, Y.-Y. Duan, X.-D. Wang, and B.-X. Wang, "Effect of nanofluids on thin film evaporation in microchannels," Journal of Nanoparticle Research, vol. 13, pp. 5033-5047, 2011.

[43] S. Betelú and J. Diez, "A two-dimensional similarity solution for capillary driven flows," Physica D, vol. 126, pp. 136-140, 1999.

[44] R. Ranjan, J. Y. Murthy, S. V. Garimella, and U. Vadakkan, "A numerical model for transport in flat heat pipes considering wick microstructure effects," International Journal of Heat and Mass Transfer, vol. 54, pp. 153-168, 2011.

[45] J. Jiang, Y.-X. Tao, and L. Byrd, "Evaporative heat transfer from thin liquid film on a heated cylinder," International Journal of Heat and Mass Transfer, vol. 43, pp. 85-99, 2000.

[46] H. K. Dhavaleswarapu, J. Y. Murthy, and S. V. Garimella, "Numerical investigation of an evaporating meniscus in a channel," International Journal of Heat and Mass Transfer, vol. 55, pp. 915-924, 2012.

[47] G. Ramon and A. Oron, "Capillary rise of a meniscus with phase change," Journal of Colloid and Interface Science, vol. 327, pp. 145-151, 2008.

[48] L. Mercury and Y. Tardy, "Negative pressure of stretched liquid water. geochemistry soil capillaries," Geochimica et Cosmochinica Acta, vol. 65, pp. 33913408, 2001.

[49] S. H. Yang, M. Nosonovsk, H. Zhang, and K.-H. Chung, "Nanoscale water capillary bridges under deeply negative pressure," Chemical Physics Letters, vol. 451, pp. 88-92, 2008.

[50] N. R. Tas, P. Mela, T. Kramer, J. Berenschot, and A. van den Berg, "Capillarity induced negative pressure of water plugs in nanochannels," Nano Letters, vol. 3, pp. 1537-1540, 2003.

[51] N. R. Tas, M. Escalante, J. W. van Honschoten, H. V. Jansen, and M. Elwenspoek, "Capillary negative pressure measured by nanochannel collapse," Langmuir, vol. 26, pp. 1473-1476, 2010.

[52] Z. Visak, L. Rebelo, and J. Szydlowski, "Achieving absolute negative pressure in liquids: Precipitation phenomena in solution," Journal of Chemical Education, vol. 79, pp. 869-873, 2002. 
[53] S. J. Henderson and R. J. Speedy, "Temperature of maximum density in water at negative pressure," Journal of Physical Chemistry, vol. 91, pp. 3062-3068, 1987.

[54] M. Matsumoto, K. Yasuoka, and Y. Kataoka, "Molecular simulation of evaporation and condensation," Fluid Phase Equilibria, vol. 104, pp. 431-439, 1995.

[55] J. Yu and H. Wang, "A molecular dynamics investigation on evaporation of thin liquid films," International Journal of Heat and Mass Transfer, vol. 55, pp. 1218-1225, 2012.

[56] T. Tsuruta, H. Tanaka, and T. Masuoka, "Condensation/evaporation coefficient and velocity distributions at liquid-vapor interface," International Journal of Heat and Mass Transfer, vol. 42, pp. 4107-4116, 1999.

[57] P. C. Wayner Jr., "Nucleation, growth and surface movement of a condensing sessile droplet," Colloids and Surfaces A: Physicochemical and Engineering Aspects, vol. 206, pp. 157-165, 2002.

[58] L. Zheng, Y.-X. Wang, J. L. Plawsky, and P. C. Wayner Jr., "Accuracy of measurements of curvature and apparent contact angle in a constrained vapor buble heat exchanger," International Journal of Heat and Mass Transfer, vol. 45, pp. 2021-2030, 2002.

[59] Q. He and K. P. Hallinan, "A new particle image velocimetry technique for three-dimensional full field fluid flow measurement in evaporating films," $E x$ perimental Thermal and Fluid Science, vol. 17, pp. 230-237, 1998.

[60] C. Buffone and K. Sefaine, "Controlling evaporative thermocapillary convection using external heating: An experimental investigation," Experimental Thermal and Fluid Science, vol. 32, pp. 1287-1300, 2008.

[61] C. Buffone and K. Sefiane, "Investigation of thermocapillary convective patterns and their role in the enhancement of evaporation from pores," International Journal of Multiphase Flow, vol. 30, pp. 1071-1091, 2004.

[62] R. Lucas, "Ueber das zeitgesetz des kapillaren aufstiegs von fl'ussigkeiten," Kolloid-Zeitschrift, vol. 23, pp. 15-22, 1918.

[63] H. Bosanquet, "On the flow of liquids into capillary tubes," Philosophical Magazine, pp. 525-531, 1923. 
[64] J. Bell and F. Cameron, "The flow of liquids through capillary spaces," Journal of Physics Chemistry, vol. 10, no. 8, pp. 593-678, 1905.

[65] E. W. Washburn, "The dynamics of capillary flow," Physical Review, vol. 17, no. 3, pp. 273-283, 1921.

[66] Y. Yamamoto, T. Ito, A. Wakimoto, and K. Katoh, "Numerical simulations of spontaneous capillary rises with very low capillary numbers using a fronttracking method combined with generalized navier boundary condition," International Journal of Multiphase Flow, vol. 51, pp. 22-32, 2013.

[67] S. Levine, J. Lowndes, E. J. Watson, and G. Neale, "A theory of capillary rise of a liquid in a vertical cylindrical tube and in a parallel-plate channel," Journal of Colloid and Interface Science, vol. 73, pp. 136-151, 1980.

[68] B. Zhmud, F. Tiberg, and K. Hallstensson, "Dynamics of capillary rise," Journal of Colloid and Interface Science, vol. 228, pp. 263-269, 2000.

[69] J. Radulovic, K. Sefiane, and M. E. Shanahan, "Capillary rise of superspreaders," Journal of Colloid and Interface Science, vol. 361, pp. 643-648, 2011.

[70] R. Rand, "The dynamics of an evaporating meniscus," Acta Mechanica, vol. 29, pp. 135-146, 1976.

[71] A. Siebold, M. Nardin, J. Schultz, A. Walliser, and M. Oppliger, "Effect of dynamic contact angle on capillary rise phenomena," Colloids and Surfaces A: Physicochemical and Engineering Aspects, vol. 161, pp. 81-87, 2000.

[72] R. Chebbi, "Dynamics of liquid penetration into capillary tubes," Journal of Colloid and Interface Science, vol. 315, pp. 255-260, 2007.

[73] B. Lavi, A. Marmur, and J. Bachmann, "Porous media characterization by the two-liquid method: Effect of dynamic contact angle and intertia," Langmuir, vol. 24, pp. 1918-1923, 2008.

[74] N. Ichikawa and Y. Satoda, "Interface dynamics of capillary flow in a tube under negligible gravity condition," Journal of Colloid and Interface Science, vol. 162, pp. 350-355, 1994.

[75] H. Xue, Z. Fang, Y. Yang, J. Huang, and L. Zhou, "Contact angle determined by spontaneous dynamic capillary rises with hydrostatic effects: Experiment and theory," Chemical Physics Letters, vol. 432, pp. 326-330, 2006. 
[76] A. Hamraoui and T. Nylander, "Analytical approach for the lucas-washburn equation," Journal of Colloid and Interface Science, vol. 250, pp. 415-421, 2002.

[77] A. Hamraoui, K. Thuresson, T. Nylander, and V. Yaminsky, "Can a dynamic contact angle be understood interms of a friction coefficient?," Journal of Colloid and Interface Science, vol. 226, pp. 199-204, 2000.

[78] C. Extrand and S. I. Moon, "Experimental measurement of forces and energies associated with capillary rise in a vertical tube," Journal of Colloid and Interface Science, vol. 407, pp. 488-492, 2013.

[79] F. Maggi and F. Alonso-Marroquin, "Multiphase capillary flows," International Journal of Multiphase Flow, vol. 42, pp. 62-73, 2012.

[80] S. Sánchez, F. Méndez, and O. Bautista, "Capillary rise in a circular tube with interfacial condensation process," International Journal of Thermal Sciences, vol. 50, pp. 2422-2429, 2011.

[81] É. Lorenceau, D. Quéré, J.-Y. Ollitrault, and C. Clanet, "Gravitational oscillations of a liquid column in a pipe," Physics of Fluids, vol. 14, pp. 1985-1992, 2002 .

[82] K. G. Kornev and A. V. Neimark, "Sponanteous penetration of liquids into capillaries and porous membranes revisted," Journal of Colloid and Interface Science, vol. 235, pp. 101-113, 2001.

[83] J. Szekely, A. Neumann, and Y. Chuang, "The rate of capillary penetration and the applicability of the washburn equation," Journal of Colloid and Interface Science, vol. 35, pp. 273-278, 1970.

[84] D. Quéré and Élie Rachaël, "Rebounds in a capillary tube," Langmuir, vol. 15, pp. 3679-3682, 1999.

[85] N. Fries and M. Dreyer, "An analytical solution of capillary rise restrained by gravity," Journal of Colloid and Interface Science, vol. 320, pp. 259-263, 2008.

[86] R. Masoodi, E. Languri, and A. Ostadhossein, "Dynamics of liquid rise in a verticle capillary tube," Journal of Colloid and Interface Science, vol. 389, 2013.

[87] A. Duarte, D. Strier, and D. Zanette, "The rise of a liquid in a capillary tube revisited: A hydrodynamic approach," American Journal of Physics, vol. 64, pp. 413-418, 1996. 
[88] N. Fries and M. Dreyer, "The transition from inertial to viscous flow in capillary rise," Journal of Colloid and Interface Science, vol. 327, pp. 125-128, 2008.

[89] N. Fries and M. Dreyer, "Dimensionless scaling methods for capillary rise," Journal of Colloid and Interface Science, vol. 338, pp. 514-518, 2009.

[90] K. G. Kornev and A. V. Neimark, "Modeling of spontaneous penetration of viscoelastic fluids and biofluids into capillaries," Journal of Colloid and Interface Science, vol. 262, pp. 253-262, 2003.

[91] R. Pit, H. Hervet, and L. Léger, "Direct experimental evidence of slip in hexadecane: Solid interfaces," Physical Review Letters, vol. 85, pp. 980-983, 2000.

[92] C. Neto, D. R. Evans, E. Bonaccurso, H.-J. Butt, and V. S. Craig, "Boundary slip in newtonian liquids: A review of experimental studies," Reports of Progress in Physics, vol. 68, pp. 2859-2897, 2005.

[93] A. Kuz'mich and V. Novikova, "Capillary rise with meniscus evaporation for $\mathrm{kn} \leq$ 0.01," Journal of Engineering Physics, vol. 57, pp. 1298-1302, 1990.

[94] M. B. Williams and S. H. Davis, "Nonlinear theory of film rupture," Journal of Colloid and Interface Science, vol. 90, pp. 220-228, 1982.

[95] S. Bankoff, "1987 max jacob memorial award lecture: Dynamics and stability of thin heated liquid films," Journal of Heat Transfer, vol. 112, pp. 538-546, 1990.

[96] J. Burelbach, S. Bankoff, and S. Davis, "Nonlinear stability of evaporating/condensing liquid films," Journal of Fluid Mechanics, vol. 195, pp. 463-494, 1988.

[97] A. Sheludko, "Thin liquid films," Advances in Colloid and Interface Science, vol. 1, pp. 391-464, 1967.

[98] A. Vrij, "Possible mechanism for the spontaneous rupture of thin, free liquid films," Discussions of the Faraday Society, vol. 42, pp. 23-33, 1966.

[99] E. Ruckenstein and R. Jain, "Spontaneous rupture of thin liquid films," Molecular and Chemical Physics, vol. 70, pp. 132-147, 1974.

[100] A. Sharma and E. Ruckenstein, "An analytical nonlinear theory of thin film rupture and its application to wetting films," Journal of Colloid and Interface Science, vol. 113, pp. 456-479, 1986. 
[101] S. Davis, "Thermocapillary instabilities," Annual Review of Fluid Mechanics, vol. 19, pp. 403-435, 1987.

[102] S. Bankhoff, "Stability of liquid flow down a heated inclined plane," International Journal of Heat and Mass Transfer, vol. 14, pp. 377-385, 1971.

[103] O. E. Shklyaev and E. Fried, "Stability of an evaporating thin liquid film," Journal of Fluid Mechanics, vol. 584, pp. 157-183, 2007.

[104] T. P. Witelski and A. J. Bernoff, "Dynamics of three-dimensional thin film rupture," Physica D, vol. 147, pp. 155-176, 2000.

[105] A. Benselama, S. Harmand, and K. Sefiane, "Thermocapillary effects on steadily evaporating contact line: A perturbative local analysis," Physics of Fluids, vol. 24, pp. 072105-1-38, 2012.

[106] A. Avramenko, I. Shevchuk, S. Harmand, and A. Tyrinov, "Thermocapillary instability in an evaporating two-dimensional thin layer film," International Journal of Heat and Mass Transfer, vol. 91, pp. 77-88, 2015.

[107] A. Oron, S. H. Davis, and S. G. Bankhoff, "Long-scale evolution of thin liquid films," Reviews of Modern Physics, vol. 69, pp. 931-980, 1997.

[108] R. Craster and O. Matar, "Dynamics and stability of thin liquid films," Reviews of Modern Physics, vol. 81, pp. 1131-1198, 2009.

[109] D. Pratt, J. Brown, and K. Hallinan, "Thermocapillary effects on the stability of a heated, curved meniscus," ASME: Journal of Heat Transfer, vol. 120, pp. 220$226,1998$.

[110] S. Harmand, K. Sefiane, N. Lancial, and A. Benselama, "Experimental and theoretical investigation of the evaporating and stability of a meniscus in a flat micro-channel," International Journal of Thermal Sciences, vol. 50, pp. 18451852, 2011.

[111] G. Preiss and P. Wayner Jr., "Evaporation from a capillary tube," ASME: Journal of Heat Transfer, vol. 98, pp. 178-181, 1976.

[112] M. Garcia, Experimental and theoretical investigation of instability within a heated meniscus. PhD thesis, Carleton University, 2013.

[113] C. Buffone, K. Sefiane, and W. Easson, "Marangoni-driven instabilities of an evaporating liquid-vapor interface," Physical Review E, vol. 71, pp. 056302-1-8, 2005. 
[114] J. Plawsky, S. Panchamgam, S. Gokhalea, P. Wayner Jr., and S. DasGupta, "A study of the oscillating corner meniscus in a vertical constrained vapor bubble system," Superlattices and Microstructures, vol. 35, pp. 559-572, 2004.

[115] D. Lide and H. Kehianian, CRC Handbook of thermophysical and thermochemical data. CRC Press, 1994.

[116] J. Polansky and T. Kaya, "An experimental and numerical study of capillary rise with evaporation," International Journal of Thermal Sciences, vol. 91, pp. 25$33,2015$.

[117] M. Unsal, "Non-linear stability of forced vapour flow condensation," International Journal of Heat and Mass Transfer, vol. 31, pp. 1619-1626, 1988.

[118] D. Pratt and K. Hallinan, "Thermocapillary effects on the wetting characteristics of a heated curved meniscus," Journal of Thermophysics and Heat Transfer, vol. 11, no. 4, 1997. 


\section{Appendix A}

\section{Velocity in $\mathrm{Y}$}

$$
V=V_{1} Y^{2}+V_{2} Y^{3}
$$

where,

$$
\begin{aligned}
V_{1}= & -1 / 2 \frac{\zeta M a E^{2} \Phi^{5} \mathcal{H}_{X X} \mathcal{H}}{\operatorname{Pe}\left(\Phi^{2}-2\right)^{2}(E \Phi \mathcal{H}+1)^{2}}+\frac{\zeta M a E^{2} \Phi^{3} \mathcal{H}_{X X} \mathcal{H}}{\operatorname{Pe}\left(\Phi^{2}-2\right)^{2}(E \Phi \mathcal{H}+1)^{2}} \\
& +2 \frac{\zeta M a E \Phi \Phi_{X} \mathcal{H}_{X}}{\operatorname{Pe}\left(\Phi^{2}-2\right)^{2}(E \Phi \mathcal{H}+1)^{2}}+1 / 2 \frac{\zeta M a E^{2} \Phi^{5} \mathcal{H}_{X}{ }^{2}}{\operatorname{Pe}\left(\Phi^{2}-2\right)^{2}(E \Phi \mathcal{H}+1)^{2}} \\
& -\frac{\zeta M a E^{2} \Phi^{3} \mathcal{H}_{X}{ }^{2}}{\operatorname{Pe}\left(\Phi^{2}-2\right)^{2}(E \Phi \mathcal{H}+1)^{2}}-1 / 2 \frac{\zeta M a E \Phi^{4} \mathcal{H}_{X X}}{\operatorname{Pe}\left(\Phi^{2}-2\right)^{2}(E \Phi \mathcal{H}+1)^{2}} \\
& +\frac{\zeta M a E \Phi^{2} \mathcal{H}_{X X}}{\operatorname{Pe}\left(\Phi^{2}-2\right)^{2}(E \Phi \mathcal{H}+1)^{2}}+1 / 2 \frac{\zeta M a E^{2} \Phi^{4} \Phi_{X} \mathcal{H}{ }_{X} \mathcal{H}}{\operatorname{Pe}\left(\Phi^{2}-2\right)^{2}(E \Phi \mathcal{H}+1)^{2}} \\
& +\frac{\zeta M a E^{2} \Phi^{2} \Phi_{X} \mathcal{H}_{X} \mathcal{H}}{P e\left(\Phi^{2}-2\right)^{2}(E \Phi \mathcal{H}+1)^{2}}
\end{aligned}
$$




$$
\begin{aligned}
& V_{2}=2 / 3 \frac{M a E \zeta \Phi_{X}^{2}}{\operatorname{Pe}\left(\Phi^{2}-2\right)^{2}(E \Phi \mathcal{H}+1)^{3}}+1 / 3 \frac{E^{3} \Phi^{6} \zeta M a \mathcal{H}_{X X} \mathcal{H}}{\operatorname{Pe}\left(\Phi^{2}-2\right)^{2}(E \Phi \mathcal{H}+1)^{3}} \\
& -2 / 3 \frac{\Phi^{4} \zeta M a E^{3} \mathcal{H}_{X X} \mathcal{H}}{\operatorname{Pe}\left(\Phi^{2}-2\right)^{2}(E \Phi \mathcal{H}+1)^{3}}+\frac{E^{2} \Phi^{3} M a \zeta \Phi_{X}{ }^{2} \mathcal{H}}{P e\left(\Phi^{2}-2\right)^{2}(E \Phi \mathcal{H}+1)^{3}} \\
& -2 / 3 \frac{M a E^{2} \zeta \Phi_{X}{ }^{2} \Phi \mathcal{H}}{\operatorname{Pe}\left(\Phi^{2}-2\right)^{2}(E \Phi \mathcal{H}+1)^{3}}+\frac{\Phi^{4} \zeta M a E^{2} \Phi_{X} \mathcal{H}_{X}}{\operatorname{Pe}\left(\Phi^{2}-2\right)^{2}(E \Phi \mathcal{H}+1)^{3}} \\
& -10 / 3 \frac{\zeta M a E^{2} \Phi_{X} \Phi^{2} \mathcal{H}_{X}}{\operatorname{Pe}\left(\Phi^{2}-2\right)^{2}(E \Phi \mathcal{H}+1)^{3}}-1 / 6 \Lambda_{X}+4 / 3 \frac{\Lambda_{X}}{\left(\Phi^{2}-2\right)^{2}(E \Phi \mathcal{H}+1)^{3}} \\
& -2 / 3 \frac{M a \Phi^{6} E^{3} \mathcal{H}_{X}^{2} \zeta}{\operatorname{Pe}\left(\Phi^{2}-2\right)^{2}(E \Phi \mathcal{H}+1)^{3}}+1 / 3 \frac{M a E \zeta \Phi_{X}^{2} \Phi^{2}}{\operatorname{Pe}\left(\Phi^{2}-2\right)^{2}(E \Phi \mathcal{H}+1)^{3}} \\
& +4 / 3 \frac{M a \Phi^{4} E^{3} \mathcal{H}_{X}^{2} \zeta}{\operatorname{Pe}\left(\Phi^{2}-2\right)^{2}(E \Phi \mathcal{H}+1)^{3}}+1 / 3 \frac{E^{2} \Phi^{5} \zeta M a \mathcal{H}_{X X}}{\operatorname{Pe}\left(\Phi^{2}-2\right)^{2}(E \Phi \mathcal{H}+1)^{3}} \\
& -2 / 3 \frac{E^{2} \Phi^{3} \zeta M a \mathcal{H}_{X X}}{\operatorname{Pe}\left(\Phi^{2}-2\right)^{2}(E \Phi \mathcal{H}+1)^{3}}+1 / 3 \frac{\mathcal{H}^{3} \Phi^{7} E^{3} \Lambda_{X}}{\left(\Phi^{2}-2\right)^{2}(E \Phi \mathcal{H}+1)^{3}} \\
& +\frac{\mathcal{H}^{2} \Phi^{6} E^{2} \Lambda_{X}}{\left(\Phi^{2}-2\right)^{2}(E \Phi \mathcal{H}+1)^{3}}-4 \frac{E \Phi^{3} \Lambda_{X} \mathcal{H}}{\left(\Phi^{2}-2\right)^{2}(E \Phi \mathcal{H}+1)^{3}} \\
& -4 \frac{\mathcal{H}^{2} \Phi^{4} E^{2} \Lambda_{X}}{\left(\Phi^{2}-2\right)^{2}(E \Phi \mathcal{H}+1)^{3}}+4 \frac{\mathcal{H}^{2} \Phi^{2} E^{2} \Lambda_{X}}{\left(\Phi^{2}-2\right)^{2}(E \Phi \mathcal{H}+1)^{3}} \\
& +\frac{E \Phi^{5} \Lambda_{X} \mathcal{H}}{\left(\Phi^{2}-2\right)^{2}(E \Phi \mathcal{H}+1)^{3}}+4 \frac{E \Lambda_{X} \Phi \mathcal{H}}{\left(\Phi^{2}-2\right)^{2}(E \Phi \mathcal{H}+1)^{3}} \\
& +4 / 3 \frac{E^{3} \Lambda_{X} \Phi^{3} \mathcal{H}^{3}}{\left(\Phi^{2}-2\right)^{2}(E \Phi \mathcal{H}+1)^{3}}-4 / 3 \frac{\mathcal{H}^{3} \Phi^{5} E^{3} \Lambda_{X}}{\left(\Phi^{2}-2\right)^{2}(E \Phi \mathcal{H}+1)^{3}} \\
& -2 / 3 \frac{E^{3} \Phi^{3} M a \zeta \Phi_{X} \mathcal{H}_{X} \mathcal{H}}{\operatorname{Pe}\left(\Phi^{2}-2\right)^{2}(E \Phi \mathcal{H}+1)^{3}}-1 / 3 \frac{E^{3} \Phi^{5} M a \zeta \Phi_{X} \mathcal{H}_{X} \mathcal{H}}{\operatorname{Pe}\left(\Phi^{2}-2\right)^{2}(E \Phi \mathcal{H}+1)^{3}} \\
& -4 / 3 \frac{\Lambda_{X} \Phi^{2}}{\left(\Phi^{2}-2\right)^{2}(E \Phi \mathcal{H}+1)^{3}}+1 / 3 \frac{\Phi^{4} \Lambda_{X}}{\left(\Phi^{2}-2\right)^{2}(E \Phi \mathcal{H}+1)^{3}}
\end{aligned}
$$




\section{Appendix B}

\section{Evolution equation}

$$
\begin{aligned}
& \mathcal{H}_{\tau}=7 / 3 \frac{\mathcal{H}^{3} \Phi^{2} \operatorname{Re} \zeta M a E^{2} \mathcal{H}_{X X}}{\operatorname{Pe}\left(\Phi^{2}-2\right)^{2}(\mathcal{H} E \Phi+1)^{4}}-1 / 3 \frac{\mathcal{H}^{4} \Phi^{5} E^{2} \operatorname{Re} \zeta^{2} \operatorname{Re}{ }_{m} \mathcal{H}_{X X}}{\left(\Phi^{2}-2\right)^{2}(\mathcal{H} E \Phi+1)^{4}} \\
& -1 / 3 \frac{\mathcal{H}^{5} \Phi^{4} R e \zeta^{2} E^{2} R e_{m} \Phi_{X X}}{\left(\Phi^{2}-2\right)^{2}(\mathcal{H} E \Phi+1)^{4}}-\frac{\mathcal{H}^{2} \Phi^{4} R e \zeta^{2} R e_{m} E \mathcal{H}_{X}{ }^{2}}{\left(\Phi^{2}-2\right)^{2}(\mathcal{H} E \Phi+1)^{4}} \\
& -1 / 3 \frac{\mathcal{H}^{3} \Phi^{4} \operatorname{Re} \zeta^{2} \operatorname{Re}_{m} E \mathcal{H}_{X X}}{\left(\Phi^{2}-2\right)^{2}(\mathcal{H} E \Phi+1)^{4}}-8 \frac{\mathcal{H}^{5} \zeta^{4} R e E^{3} \mathcal{H}_{X X X} \mathcal{H}_{X}}{C a\left(\Phi^{2}-2\right)^{2}(\mathcal{H} E \Phi+1)^{4} \Phi} \\
& -32 \frac{\mathcal{H}^{4} \operatorname{Re} \zeta^{4} E \mathcal{H}_{X X} \Phi_{X}{ }^{2}}{C a\left(\Phi^{2}-2\right)^{2}(\mathcal{H} E \Phi+1)^{4} \Phi^{5}}+6 \frac{\mathcal{H}^{2} \operatorname{Re} \zeta^{4} \mathcal{H}_{X X} \mathcal{H}_{X} \Phi_{X}}{C a\left(\Phi^{2}-2\right)^{2}(\mathcal{H} E \Phi+1)^{4} \Phi^{5}} \\
& -1 / 6 \frac{\mathcal{H}^{4} \Phi^{6} E^{4} \operatorname{Re} \zeta M a \mathcal{H}_{X}{ }^{2}}{\operatorname{Pe}\left(\Phi^{2}-2\right)^{2}(\mathcal{H} E \Phi+1)^{4}}-1 / 6 \frac{\mathcal{H}^{5} \Phi^{6} E^{4} \operatorname{Re} \zeta M a \mathcal{H}_{X X}}{\operatorname{Pe}\left(\Phi^{2}-2\right)^{2}(\mathcal{H} E \Phi+1)^{4}} \\
& -2 / 3 \frac{\mathcal{H}^{3} \Phi^{5} E^{3} \operatorname{Re} \zeta M a \mathcal{H}_{X}{ }^{2}}{\operatorname{Pe}\left(\Phi^{2}-2\right)^{2}(\mathcal{H} E \Phi+1)^{4}}-5 / 6 \frac{\mathcal{H}^{4} \Phi^{5} E^{3} \operatorname{Re} \zeta M a \mathcal{H}_{X X}}{\operatorname{Pe}\left(\Phi^{2}-2\right)^{2}(\mathcal{H E} \Phi+1)^{4}} \\
& -1 / 3 \frac{\mathcal{H}^{5} \Phi^{4} \operatorname{Re} \zeta E^{3} M a \Phi_{X X}}{\operatorname{Pe}\left(\Phi^{2}-2\right)^{2}(\mathcal{H} E \Phi+1)^{4}}+1 / 3 \frac{\mathcal{H}^{4} \Phi^{4} \operatorname{Re} \zeta E^{4} M a \mathcal{H}_{X}{ }^{2}}{\operatorname{Pe}\left(\Phi^{2}-2\right)^{2}(\mathcal{H} E \Phi+1)^{4}} \\
& -3 / 2 \frac{\mathcal{H}^{2} \Phi^{4} \operatorname{Re} \zeta M a E^{2} \mathcal{H}_{X}{ }^{2}}{\operatorname{Pe}\left(\Phi^{2}-2\right)^{2}(\mathcal{H} E \Phi+1)^{4}}-1 / 2 \frac{\mathcal{H}^{6} \Phi^{4} \operatorname{Re} \zeta^{4} E^{4} \mathcal{H}_{X X X} \mathcal{H}_{X}}{\operatorname{Ca}\left(\Phi^{2}-2\right)^{2}(\mathcal{H E} \Phi+1)^{4}} \\
& -7 / 6 \frac{\mathcal{H}^{3} \Phi^{4} \operatorname{Re} \zeta M a E^{2} \mathcal{H}_{X X}}{\operatorname{Pe}\left(\Phi^{2}-2\right)^{2}(\mathcal{H} E \Phi+1)^{4}}+1 / 3 \frac{\mathcal{H}^{5} \Phi^{4} \operatorname{Re} \zeta E^{4} M a \mathcal{H}_{X X}}{\operatorname{Pe}\left(\Phi^{2}-2\right)^{2}(\mathcal{H} E \Phi+1)^{4}} \\
& +\frac{\mathcal{H}^{2} \Phi \operatorname{Re} \zeta M a E \mathcal{H}_{X X}}{\operatorname{Pe}\left(\Phi^{2}-2\right)^{2}(\mathcal{H} E \Phi+1)^{4}}+2 \frac{\mathcal{H} E \Phi \operatorname{Re} \zeta M a \mathcal{H}_{X}{ }^{2}}{\operatorname{Pe}\left(\Phi^{2}-2\right)^{2}(\mathcal{H} E \Phi+1)^{4}}
\end{aligned}
$$




$$
\begin{aligned}
& +8 \frac{\mathcal{H}^{5} \Phi \operatorname{Re} \zeta^{4} E^{3} \mathcal{H}_{X X X} \mathcal{H}_{X}}{C a\left(\Phi^{2}-2\right)^{2}(\mathcal{H} E \Phi+1)^{4}}-2 \frac{\mathcal{H}^{3} \Phi \operatorname{Re} \zeta^{4} E \mathcal{H}_{X X X} \mathcal{H}_{X}}{C a\left(\Phi^{2}-2\right)^{2}(\mathcal{H} E \Phi+1)^{4}} \\
& +1 / 3 \frac{\mathcal{H}^{3} \Phi \operatorname{Re} \zeta M a E \Phi_{X}{ }^{2}}{\operatorname{Pe}\left(\Phi^{2}-2\right)^{2}(\mathcal{H} E \Phi+1)^{4}}-8 \frac{\mathcal{H}^{6} \Phi \operatorname{Re} \zeta^{4} E^{3} \mathcal{H}_{X X} \Phi_{X}{ }^{2}}{C a\left(\Phi^{2}-2\right)^{2}(\mathcal{H} E \Phi+1)^{4}} \\
& -2 / 3 \frac{\mathcal{H}^{5} \Phi \operatorname{Re} \zeta E^{3} M a \Phi_{X}{ }^{2}}{\operatorname{Pe}\left(\Phi^{2}-2\right)^{2}(\mathcal{H} E \Phi+1)^{4}}-2 \frac{\mathcal{H}^{7} \Phi \operatorname{Re} \zeta^{4} E^{4} \mathcal{H}_{X X} \Phi_{X X}}{C a\left(\Phi^{2}-2\right)^{2}(\mathcal{H} E \Phi+1)^{4}} \\
& +3 \frac{\mathcal{H}^{5} \Phi \operatorname{Re} \zeta^{4} E^{2} \mathcal{H}_{X X} \Phi_{X X}}{C a\left(\Phi^{2}-2\right)^{2}(\mathcal{H} E \Phi+1)^{4}}+4 / 3 \frac{\mathcal{H}^{4} \Phi \operatorname{Re} \zeta E^{2} M a \Phi_{X X}}{\operatorname{Pe}\left(\Phi^{2}-2\right)^{2}(\mathcal{H} E \Phi+1)^{4}} \\
& -4 \frac{\mathcal{H}^{7} \Phi \operatorname{Re} \zeta^{4} E^{4} \mathcal{H}_{X X X} \Phi_{X}}{C a\left(\Phi^{2}-2\right)^{2}(\mathcal{H} E \Phi+1)^{4}}+6 \frac{\mathcal{H}^{5} \Phi \operatorname{Re} \zeta^{4} E^{2} \mathcal{H}_{X X X} \Phi_{X}}{C a\left(\Phi^{2}-2\right)^{2}(\mathcal{H} E \Phi+1)^{4}} \\
& +12 \frac{\mathcal{H}^{4} \operatorname{Re} \zeta^{4} E^{2} \mathcal{H}_{X X X} \mathcal{H}_{X}}{C a\left(\Phi^{2}-2\right)^{2}(\mathcal{H} E \Phi+1)^{4}}+48 \frac{\mathcal{H}^{5} \zeta^{4} \operatorname{Re}^{2} \mathcal{H}_{X X} \Phi_{X}{ }^{2}}{C a\left(\Phi^{2}-2\right)^{2}(\mathcal{H} E \Phi+1)^{4} \Phi^{2}} \\
& -8 \frac{\mathcal{H}^{7} \operatorname{Re} \zeta^{4} E^{4} \mathcal{H}_{X X} \Phi_{X}{ }^{2}}{C a\left(\Phi^{2}-2\right)^{2}(\mathcal{H} E \Phi+1)^{4} \Phi^{2}}-16 \frac{\mathcal{H}^{4} \operatorname{Re} \zeta^{4} E \mathcal{H}_{X X X} \Phi_{X}}{C a\left(\Phi^{2}-2\right)^{2}(\mathcal{H} E \Phi+1)^{4} \Phi^{2}} \\
& +8 \frac{\mathcal{H}^{6} \operatorname{Re}^{4} E^{3} \mathcal{H}_{X X} \Phi_{X X}}{C a\left(\Phi^{2}-2\right)^{2}(\mathcal{H} E \Phi+1)^{4} \Phi^{2}}-8 \frac{\mathcal{H}^{4} \operatorname{Re} \zeta^{4} E \mathcal{H}_{X X} \Phi_{X X}}{C a\left(\Phi^{2}-2\right)^{2}(\mathcal{H} E \Phi+1)^{4} \Phi^{2}} \\
& +16 \frac{\mathcal{H}^{6} \operatorname{Re} \zeta^{4} E^{3} \mathcal{H}_{X X X} \Phi_{X}}{C a\left(\Phi^{2}-2\right)^{2}(\mathcal{H} E \Phi+1)^{4} \Phi^{2}}-12 \frac{\mathcal{H}^{4} \operatorname{Re} \zeta^{4} E^{2} \mathcal{H}_{X X X} \mathcal{H}_{X}}{C a\left(\Phi^{2}-2\right)^{2}(\mathcal{H} E \Phi+1)^{4} \Phi^{2}} \\
& -2 \frac{\mathcal{H}^{7} \Phi^{2} \operatorname{Re} \zeta^{4} E^{4} \mathcal{H}_{X X} \Phi_{X}{ }^{2}}{C a\left(\Phi^{2}-2\right)^{2}(\mathcal{H} E \Phi+1)^{4}}-1 / 3 \frac{\mathcal{H}^{3} \Phi^{2} \operatorname{Re} \zeta M a E \Phi_{X X}}{\operatorname{Pe}\left(\Phi^{2}-2\right)^{2}(\mathcal{H} E \Phi+1)^{4}} \\
& +4 / 3 \frac{\mathcal{H}^{4} \Phi^{2} \operatorname{Re} \zeta E^{2} M a \Phi_{X}{ }^{2}}{\operatorname{Pe}\left(\Phi^{2}-2\right)^{2}(\mathcal{H} E \Phi+1)^{4}}+2 \frac{\mathcal{H}^{6} \Phi^{2} \operatorname{Re} \zeta^{4} E^{3} \mathcal{H}_{X X} \Phi_{X X}}{C a\left(\Phi^{2}-2\right)^{2}(\mathcal{H} E \Phi+1)^{4}} \\
& +4 \frac{\mathcal{H}^{6} \Phi^{2} \operatorname{Re} \zeta^{4} E^{3} \mathcal{H}_{X X X} \Phi_{X}}{C a\left(\Phi^{2}-2\right)^{2}(\mathcal{H} E \Phi+1)^{4}}-3 \frac{\mathcal{H}^{4} \Phi^{2} \operatorname{Re} \zeta^{4} E^{2} \mathcal{H}_{X X X} \mathcal{H}_{X}}{C a\left(\Phi^{2}-2\right)^{2}(\mathcal{H} E \Phi+1)^{4}} \\
& +2 / 3 \frac{\mathcal{H}^{5} \Phi^{2} \operatorname{Re} \zeta E^{3} M a \Phi_{X X}}{\operatorname{Pe}\left(\Phi^{2}-2\right)^{2}(\mathcal{H} E \Phi+1)^{4}}+3 \frac{\mathcal{H}^{2} \Phi^{2} \operatorname{Re} \zeta M a E^{2} \mathcal{H}_{X}{ }^{2}}{\operatorname{Pe}\left(\Phi^{2}-2\right)^{2}(\mathcal{H} E \Phi+1)^{4}} \\
& +2 \frac{\mathcal{H}^{6} \Phi^{2} \operatorname{Re} \zeta^{4} E^{4} \mathcal{H}_{X X X} \mathcal{H}_{X}}{C a\left(\Phi^{2}-2\right)^{2}(\mathcal{H} E \Phi+1)^{4}}-4 \frac{\mathcal{H}^{2} \operatorname{Re} \zeta^{2} \operatorname{Re}_{m} E \mathcal{H}_{X}{ }^{2}}{\left(\Phi^{2}-2\right)^{2}(\mathcal{H} E \Phi+1)^{4}} \\
& -4 / 3 \frac{\mathcal{H}^{3} R e \zeta^{2} \operatorname{Re}_{m} E \mathcal{H}_{X X}}{\left(\Phi^{2}-2\right)^{2}(\mathcal{H} E \Phi+1)^{4}}+4 \frac{\mathcal{H}^{5} \zeta^{2} R e E^{2} R e_{m} \Phi_{X}{ }^{2}}{\left(\Phi^{2}-2\right)^{2}(\mathcal{H} E \Phi+1)^{4} \Phi}
\end{aligned}
$$




$$
\begin{aligned}
& -4 / 3 \frac{\mathcal{H}^{4} \zeta^{2} \operatorname{ReERe}_{m} \Phi_{X X}}{\left(\Phi^{2}-2\right)^{2}(\mathcal{H} E \Phi+1)^{4} \Phi}-2 / 3 \frac{\mathcal{H}^{4} \Phi^{3} \operatorname{Re} \zeta E^{2} M a \Phi_{X X}}{\operatorname{Pe}\left(\Phi^{2}-2\right)^{2}(\mathcal{H} E \Phi+1)^{4}} \\
& +\frac{\mathcal{H}^{7} \Phi^{3} \operatorname{Re} \zeta^{4} E^{4} \mathcal{H}_{X X X} \Phi_{X}}{C a\left(\Phi^{2}-2\right)^{2}(\mathcal{H} E \Phi+1)^{4}}-1 / 2 \frac{\mathcal{H}^{2} \Phi^{3} \operatorname{Re} \zeta M a E \mathcal{H}_{X X}}{\operatorname{Pe}\left(\Phi^{2}-2\right)^{2}(\mathcal{H} E \Phi+1)^{4}} \\
& -\frac{\mathcal{H} \Phi^{3} E \operatorname{Re} \zeta M a \mathcal{H}_{X}{ }^{2}}{\operatorname{Pe}\left(\Phi^{2}-2\right)^{2}(\mathcal{H} E \Phi+1)^{4}}-2 \frac{\mathcal{H}^{5} \Phi^{3} \operatorname{Re} \zeta^{4} E^{3} \mathcal{H}_{X X X} \mathcal{H}_{X}}{C a\left(\Phi^{2}-2\right)^{2}(\mathcal{H} E \Phi+1)^{4}} \\
& +5 / 3 \frac{\mathcal{H}^{4} \Phi^{3} \operatorname{Re} \zeta E^{3} M a \mathcal{H}_{X X}}{\operatorname{Pe}\left(\Phi^{2}-2\right)^{2}(\mathcal{H} E \Phi+1)^{4}}+4 / 3 \frac{\mathcal{H}^{3} \Phi^{3} \operatorname{Re} \zeta M a E^{3} \mathcal{H}_{X}{ }^{2}}{\operatorname{Pe}\left(\Phi^{2}-2\right)^{2}(\mathcal{H} E \Phi+1)^{4}} \\
& +\frac{\mathcal{H}^{5} \Phi^{3} \operatorname{Re} \zeta E^{3} M a \Phi_{X}{ }^{2}}{\operatorname{Pe}\left(\Phi^{2}-2\right)^{2}(\mathcal{H} E \Phi+1)^{4}}+1 / 2 \frac{\mathcal{H}^{7} \Phi^{3} \operatorname{Re} \zeta^{4} E^{4} \mathcal{H}_{X X} \Phi_{X X}}{C a\left(\Phi^{2}-2\right)^{2}(\mathcal{H} E \Phi+1)^{4}} \\
& -48 \frac{\mathcal{H}^{5} \zeta^{4} \operatorname{Re} E^{2} \mathcal{H}_{X X} \Phi_{X}{ }^{2}}{C a\left(\Phi^{2}-2\right)^{2}(\mathcal{H} E \Phi+1)^{4} \Phi^{4}}+8 \frac{\mathcal{H}^{4} \operatorname{Re} \zeta^{4} E \mathcal{H}_{X X} \Phi_{X X}}{C a\left(\Phi^{2}-2\right)^{2}(\mathcal{H} E \Phi+1)^{4} \Phi^{4}} \\
& +16 \frac{\mathcal{H}^{4} \operatorname{Re}^{4} E \mathcal{H}_{X X X} \Phi_{X}}{C a\left(\Phi^{2}-2\right)^{2}(\mathcal{H} E \Phi+1)^{4} \Phi^{4}}+4 / 3 \frac{\mathcal{H}^{5} \Phi^{2} R e \zeta^{2} E^{2} R e_{m} \Phi_{X X}}{\left(\Phi^{2}-2\right)^{2}(\mathcal{H} E \Phi+1)^{4}} \\
& +4 \frac{\mathcal{H}^{2} \Phi^{2} R e \zeta^{2} R e_{m} E \mathcal{H}_{X}{ }^{2}}{\left(\Phi^{2}-2\right)^{2}(\mathcal{H} E \Phi+1)^{4}}+4 / 3 \frac{\mathcal{H}^{3} \Phi^{2} R e \zeta^{2} R e_{m} E \mathcal{H}_{X X}}{\left(\Phi^{2}-2\right)^{2}(\mathcal{H} E \Phi+1)^{4}} \\
& +4 / 3 \frac{\mathcal{H}^{4} \Phi R e \zeta^{2} E R e_{m} \Phi_{X X}}{\left(\Phi^{2}-2\right)^{2}(\mathcal{H} E \Phi+1)^{4}}-4 \frac{\mathcal{H}^{5} \Phi R e \zeta^{2} E^{2} R e_{m} \Phi_{X}{ }^{2}}{\left(\Phi^{2}-2\right)^{2}(\mathcal{H} E \Phi+1)^{4}} \\
& -4 / 3 \frac{\mathcal{H}^{4} \Phi R e \zeta^{2} E^{2} \operatorname{Re}_{m} \mathcal{H}_{X X}}{\left(\Phi^{2}-2\right)^{2}(\mathcal{H} E \Phi+1)^{4}}-12 \frac{\mathcal{H}^{5} \zeta^{4} \operatorname{Re}^{2} \mathcal{H}_{X X} \Phi_{X}{ }^{2}}{C a\left(\Phi^{2}-2\right)^{2}(\mathcal{H} E \Phi+1)^{4}} \\
& +8 \frac{\mathcal{H}^{7} \operatorname{Re}^{4} E^{4} \mathcal{H}_{X X} \Phi_{X}{ }^{2}}{\operatorname{Ca}\left(\Phi^{2}-2\right)^{2}(\mathcal{H} E \Phi+1)^{4}}+2 / 3 \frac{\mathcal{H}^{3} \operatorname{Re} \zeta M a E \Phi_{X X}}{\operatorname{Pe}\left(\Phi^{2}-2\right)^{2}(\mathcal{H} E \Phi+1)^{4}} \\
& +4 \frac{\mathcal{H}^{4} \operatorname{Re} \zeta^{4} E \mathcal{H}_{X X X} \Phi_{X}}{C a\left(\Phi^{2}-2\right)^{2}(\mathcal{H} E \Phi+1)^{4}}-8 \frac{\mathcal{H}^{6} \operatorname{Re} \zeta^{4} E^{3} \mathcal{H}_{X X} \Phi_{X X}}{C a\left(\Phi^{2}-2\right)^{2}(\mathcal{H} E \Phi+1)^{4}} \\
& +2 \frac{\mathcal{H}^{4} \operatorname{Re} \zeta^{4} E \mathcal{H}_{X X} \Phi_{X X}}{C a\left(\Phi^{2}-2\right)^{2}(\mathcal{H} E \Phi+1)^{4}}-16 \frac{\mathcal{H}^{6} \operatorname{Re}^{4} E^{3} \mathcal{H}_{X X X} \Phi_{X}}{C a\left(\Phi^{2}-2\right)^{2}(\mathcal{H} E \Phi+1)^{4}} \\
& -2 \frac{\mathcal{H}^{6} \operatorname{Re} \zeta^{4} E^{4} \mathcal{H}_{X X X} \mathcal{H}_{X}}{C a\left(\Phi^{2}-2\right)^{2}(\mathcal{H} E \Phi+1)^{4}}-1 / 3 \frac{\mathcal{H}^{4} \Phi^{3} \operatorname{Re} \zeta^{2} E R e_{m} \Phi_{X X}}{\left(\Phi^{2}-2\right)^{2}(\mathcal{H} E \Phi+1)^{4}} \\
& +\frac{\mathcal{H}^{5} \Phi^{3} \operatorname{Re} \zeta^{2} E^{2} \operatorname{Re}_{m} \Phi_{X}{ }^{2}}{\left(\Phi^{2}-2\right)^{2}(\mathcal{H} E \Phi+1)^{4}}+4 / 3 \frac{\mathcal{H}^{4} \Phi^{3} \operatorname{Re} \zeta^{2} E^{2} \operatorname{Re}_{m} \mathcal{H}_{X X}}{\left(\Phi^{2}-2\right)^{2}(\mathcal{H} E \Phi+1)^{4}}
\end{aligned}
$$




$$
\begin{aligned}
& +32 \frac{\mathcal{H}^{6} \operatorname{Re}^{4} E^{3} \mathcal{H}_{X X} \Phi_{X}{ }^{2}}{\operatorname{Ca}\left(\Phi^{2}-2\right)^{2}(\mathcal{H} E \Phi+1)^{4} \Phi}+2 / 3 \frac{\mathcal{H}^{3} \operatorname{Re} \zeta M a E \Phi_{X}{ }^{2}}{\operatorname{Pe}\left(\Phi^{2}-2\right)^{2}(\mathcal{H} E \Phi+1)^{4} \Phi} \\
& -24 \frac{\mathcal{H}^{5} \zeta^{4} \operatorname{Re} E^{2} \mathcal{H}_{X X X} \Phi_{X}}{C a\left(\Phi^{2}-2\right)^{2}(\mathcal{H} E \Phi+1)^{4} \Phi}+2 \frac{\mathcal{H}^{7} \operatorname{Re}^{4} E^{4} \mathcal{H}_{X X} \Phi_{X X}}{C a\left(\Phi^{2}-2\right)^{2}(\mathcal{H} E \Phi+1)^{4} \Phi} \\
& -12 \frac{\mathcal{H}^{5} \zeta^{4} R e E^{2} \mathcal{H}_{X X} \Phi_{X X}}{C a\left(\Phi^{2}-2\right)^{2}(\mathcal{H} E \Phi+1)^{4} \Phi}-8 \frac{\mathcal{H}^{4} R e \zeta^{4} E \mathcal{H}_{X X} \Phi_{X}{ }^{2}}{C a\left(\Phi^{2}-2\right)^{2}(\mathcal{H} E \Phi+1)^{4} \Phi} \\
& +3 / 2 \frac{\mathcal{H}^{2} \operatorname{Re} \zeta^{4} \mathcal{H}_{X X} \mathcal{H}_{X} \Phi_{X}}{C a\left(\Phi^{2}-2\right)^{2}(\mathcal{H} E \Phi+1)^{4} \Phi}+4 \frac{\mathcal{H}^{7} \operatorname{Re} \zeta^{4} E^{4} \mathcal{H}_{X X X} \Phi_{X}}{C a\left(\Phi^{2}-2\right)^{2}(\mathcal{H} E \Phi+1)^{4} \Phi} \\
& +8 \frac{\mathcal{H}^{3} \zeta^{4} \operatorname{Re} E \mathcal{H}_{X X X} \mathcal{H}_{X}}{C a\left(\Phi^{2}-2\right)^{2}(\mathcal{H E} \Phi+1)^{4} \Phi}-6 \frac{\mathcal{H}^{2} \operatorname{Re} \zeta^{4} \mathcal{H}_{X X} \mathcal{H}_{X} \Phi_{X}}{C a\left(\Phi^{2}-2\right)^{2}(\mathcal{H} E \Phi+1)^{4} \Phi^{3}} \\
& +24 \frac{\mathcal{H}^{5} \zeta^{4} R e E^{2} \mathcal{H}_{X X X} \Phi_{X}}{C a\left(\Phi^{2}-2\right)^{2}(\mathcal{H} E \Phi+1)^{4} \Phi^{3}}+32 \frac{\mathcal{H}^{4} R e \zeta^{4} E \mathcal{H}_{X X} \Phi_{X}{ }^{2}}{C a\left(\Phi^{2}-2\right)^{2}(\mathcal{H} E \Phi+1)^{4} \Phi^{3}} \\
& -32 \frac{\mathcal{H}^{6} \operatorname{Re} \zeta^{4} E^{3} \mathcal{H}_{X X} \Phi_{X}{ }^{2}}{C a\left(\Phi^{2}-2\right)^{2}(\mathcal{H} E \Phi+1)^{4} \Phi^{3}}+12 \frac{\mathcal{H}^{5} \zeta^{4} \operatorname{Re}^{2} \mathcal{H}_{X X} \Phi_{X X}}{C a\left(\Phi^{2}-2\right)^{2}(\mathcal{H} E \Phi+1)^{4} \Phi^{3}} \\
& -8 \frac{\mathcal{H}^{3} \zeta^{4} \operatorname{Re} E \mathcal{H}_{X X X} \mathcal{H}_{X}}{C a\left(\Phi^{2}-2\right)^{2}(\mathcal{H} E \Phi+1)^{4} \Phi^{3}}-4 / 3 \frac{\mathcal{H}^{5} \zeta^{2} \operatorname{Re}^{2} \operatorname{Re}_{m} \Phi_{X X}}{\left(\Phi^{2}-2\right)^{2}(\mathcal{H} E \Phi+1)^{4}} \\
& -8 \frac{\mathcal{H} \Phi^{2} E^{3} \zeta \Gamma \mathcal{H}_{X}{ }^{2}}{\left(\Phi^{2}-2\right)^{2}(\mathcal{H} E \Phi+1)^{4}}+4 \frac{\mathcal{H}^{3} \zeta^{4} \operatorname{Re} \mathcal{H}_{X X X} \Phi_{X}}{C a\left(\Phi^{2}-2\right)^{2}(\mathcal{H} E \Phi+1)^{4} \Phi^{5}} \\
& +2 \frac{\mathcal{H}^{3} \zeta^{4} \operatorname{Re} \mathcal{H}_{X X} \Phi_{X X}}{C a\left(\Phi^{2}-2\right)^{2}(\mathcal{H} E \Phi+1)^{4} \Phi^{5}}+1 / 2 \frac{\Phi^{3} \zeta \Gamma \mathcal{H}_{X X}}{\left(\Phi^{2}-2\right)^{2}(\mathcal{H} E \Phi+1)^{4} \mathcal{H}} \\
& -2 \frac{\Phi \zeta \Gamma \mathcal{H}_{X X}}{\left(\Phi^{2}-2\right)^{2}(\mathcal{H} E \Phi+1)^{4} \mathcal{H}}-2 \frac{\zeta \Gamma \mathcal{H}_{X}{ }^{2}}{\left(\Phi^{2}-2\right)^{2}(\mathcal{H} E \Phi+1)^{4} \mathcal{H}^{2} \Phi} \\
& +2 \frac{\zeta \Gamma \mathcal{H}_{X X}}{\left(\Phi^{2}-2\right)^{2}(\mathcal{H} E \Phi+1)^{4} \mathcal{H} \Phi}-1 / 2 \frac{\Phi^{3} \zeta \Gamma \mathcal{H}_{X}{ }^{2}}{\left(\Phi^{2}-2\right)^{2}(\mathcal{H} E \Phi+1)^{4} \mathcal{H}^{2}} \\
& +2 \frac{\Phi \zeta \Gamma \mathcal{H}_{X}{ }^{2}}{\left(\Phi^{2}-2\right)^{2}(\mathcal{H} E \Phi+1)^{4} \mathcal{H}^{2}}-1 / 6 \frac{\mathcal{H}^{3} \zeta^{4} \operatorname{Re} \mathcal{H}_{X X X X}}{C a\left(\Phi^{2}-2\right)^{2}(\mathcal{H} E \Phi+1)^{4}} \\
& +2 / 3 \frac{\mathcal{H}^{3} \zeta^{4} \operatorname{Re} \mathcal{H}_{X X X X}}{C a\left(\Phi^{2}-2\right)^{2}(\mathcal{H} E \Phi+1)^{4} \Phi^{2}}-2 / 3 \frac{\mathcal{H}^{3} \zeta^{4} \operatorname{Re} \mathcal{H}_{X X X X}}{C a\left(\Phi^{2}-2\right)^{2}(\mathcal{H E} \Phi+1)^{4} \Phi^{4}} \\
& -36 \frac{\mathcal{H}^{4} \operatorname{Re} \zeta^{4} E^{2} \mathcal{H}_{X X} \mathcal{H}_{X} \Phi_{X}}{C a\left(\Phi^{2}-2\right)^{2}(\mathcal{H} E \Phi+1)^{4} \Phi}+6 \frac{\mathcal{H}^{6} \operatorname{Re} \zeta^{4} E^{4} \mathcal{H}_{X X} \mathcal{H}_{X} \Phi_{X}}{C a\left(\Phi^{2}-2\right)^{2}(\mathcal{H} E \Phi+1)^{4} \Phi}
\end{aligned}
$$




$$
\begin{aligned}
& +24 \frac{\mathcal{H}^{5} \zeta^{4} \operatorname{Re}^{3} \mathcal{H}_{X X} \mathcal{H}_{X} \Phi_{X}}{C a\left(\Phi^{2}-2\right)^{2}(\mathcal{H} E \Phi+1)^{4} \Phi^{2}}-24 \frac{\mathcal{H}^{3} \zeta^{4} \operatorname{Re} E \mathcal{H}_{X X} \mathcal{H}_{X} \Phi_{X}}{C a\left(\Phi^{2}-2\right)^{2}(\mathcal{H} E \Phi+1)^{4} \Phi^{2}} \\
& +\frac{20}{3} \frac{\mathcal{H}^{3} \Phi R e \zeta^{2} R e_{m} E \mathcal{H}_{X} \Phi_{X}}{\left(\Phi^{2}-2\right)^{2}(\mathcal{H} E \Phi+1)^{4}}+4 / 3 \frac{\mathcal{H}^{4} \zeta^{2} R e E^{2} \operatorname{Re}_{m} \mathcal{H}_{X} \Phi_{X}}{\left(\Phi^{2}-2\right)^{2}(\mathcal{H} E \Phi+1)^{4}} \\
& +6 \frac{\mathcal{H}^{5} \Phi^{2} \operatorname{Re} \zeta^{4} E^{3} \mathcal{H}_{X X} \mathcal{H}_{X} \Phi_{X}}{C a\left(\Phi^{2}-2\right)^{2}(\mathcal{H E} \Phi+1)^{4}}-\frac{\mathcal{H}^{2} \Phi^{2} \operatorname{Re} \zeta M a E \mathcal{H}_{X} \Phi_{X}}{\operatorname{Pe}\left(\Phi^{2}-2\right)^{2}(\mathcal{H} E \Phi+1)^{4}} \\
& +2 \frac{\mathcal{H}^{4} \Phi^{2} \operatorname{Re} \zeta E^{3} M a \mathcal{H}_{X} \Phi_{X}}{\operatorname{Pe}\left(\Phi^{2}-2\right)^{2}(\mathcal{H} E \Phi+1)^{4}}-6 \frac{\mathcal{H}^{6} \Phi \operatorname{Re} \zeta^{4} E^{4} \mathcal{H}_{X X} \mathcal{H}_{X} \Phi_{X}}{C a\left(\Phi^{2}-2\right)^{2}(\mathcal{H} E \Phi+1)^{4}} \\
& +9 \frac{\mathcal{H}^{4} \Phi \operatorname{Re} \zeta^{4} E^{2} \mathcal{H}_{X X} \mathcal{H}_{X} \Phi_{X}}{C a\left(\Phi^{2}-2\right)^{2}(\mathcal{H} E \Phi+1)^{4}}+\frac{17}{3} \frac{\mathcal{H}^{3} \Phi \operatorname{Re} \zeta M a E^{2} \mathcal{H}_{X} \Phi_{X}}{\operatorname{Pe}\left(\Phi^{2}-2\right)^{2}(\mathcal{H} E \Phi+1)^{4}} \\
& +3 / 2 \frac{\mathcal{H}^{6} \Phi^{3} \operatorname{Re} \zeta^{4} E^{4} \mathcal{H}_{X X} \mathcal{H}_{X} \Phi_{X}}{C a\left(\Phi^{2}-2\right)^{2}(\mathcal{H} E \Phi+1)^{4}}+1 / 3 \frac{\mathcal{H}^{5} \Phi^{3} \operatorname{Re} \zeta E^{4} M a \mathcal{H}_{X} \Phi_{X}}{\operatorname{Pe}\left(\Phi^{2}-2\right)^{2}(\mathcal{H} E \Phi+1)^{4}} \\
& -1 / 2 \frac{\mathcal{H}^{3} \Phi^{3} \operatorname{Re} \zeta M a E^{2} \mathcal{H}_{X} \Phi_{X}}{\operatorname{Pe}\left(\Phi^{2}-2\right)^{2}(\mathcal{H} E \Phi+1)^{4}}+36 \frac{\mathcal{H}^{4} \operatorname{Re} \zeta^{4} E^{2} \mathcal{H}_{X X} \mathcal{H}_{X} \Phi_{X}}{C a\left(\Phi^{2}-2\right)^{2}(\mathcal{H} E \Phi+1)^{4} \Phi^{3}} \\
& -\frac{20}{3} \frac{\mathcal{H}^{3} \operatorname{Re} \zeta^{2} \operatorname{Re}_{m} E \mathcal{H}_{X} \Phi_{X}}{\left(\Phi^{2}-2\right)^{2}(\mathcal{H} E \Phi+1)^{4} \Phi}+1 / 3 \frac{\mathcal{H}^{4} \Phi^{4} R e \zeta^{2} E^{2} R e_{m} \mathcal{H}_{X} \Phi_{X}}{\left(\Phi^{2}-2\right)^{2}(\mathcal{H} E \Phi+1)^{4}} \\
& +24 \frac{\mathcal{H}^{3} \zeta^{4} \operatorname{Re} E \mathcal{H}_{X X} \mathcal{H}_{X} \Phi_{X}}{C a\left(\Phi^{2}-2\right)^{2}(\mathcal{H} E \Phi+1)^{4} \Phi^{4}}+1 / 6 \frac{\mathcal{H}^{5} \Phi^{5} E^{4} \operatorname{Re} \zeta M a \mathcal{H}_{X} \Phi_{X}}{\operatorname{Pe}\left(\Phi^{2}-2\right)^{2}(\mathcal{H} E \Phi+1)^{4}} \\
& +2 / 3 \frac{\mathcal{H}^{4} \Phi^{4} \operatorname{Re} \zeta E^{3} M a \mathcal{H}_{X} \Phi_{X}}{\operatorname{Pe}\left(\Phi^{2}-2\right)^{2}(\mathcal{H} E \Phi+1)^{4}}-4 / 3 \frac{\mathcal{H}^{4} \Phi^{2} \operatorname{Re} \zeta^{2} E^{2} \operatorname{Re}{ }_{m} \mathcal{H}_{X} \Phi_{X}}{\left(\Phi^{2}-2\right)^{2}(\mathcal{H} E \Phi+1)^{4}} \\
& -5 / 3 \frac{\mathcal{H}^{3} \Phi^{3} \operatorname{Re}^{2} \operatorname{Re}_{m} E \mathcal{H}_{X} \Phi_{X}}{\left(\Phi^{2}-2\right)^{2}(\mathcal{H} E \Phi+1)^{4}}-24 \frac{\mathcal{H}^{5} \zeta^{4} \operatorname{Re} E^{3} \mathcal{H}_{X X} \mathcal{H}_{X} \Phi_{X}}{C a\left(\Phi^{2}-2\right)^{2}(\mathcal{H} E \Phi+1)^{4}} \\
& +6 \frac{\mathcal{H}^{3} \zeta^{4} \operatorname{Re} E \mathcal{H}_{X X} \mathcal{H}_{X} \Phi_{X}}{C a\left(\Phi^{2}-2\right)^{2}(\mathcal{H} E \Phi+1)^{4}}+4 \frac{\mathcal{H}^{2} \operatorname{Re} \zeta M a E \mathcal{H}_{X} \Phi_{X}}{\operatorname{Pe}\left(\Phi^{2}-2\right)^{2}(\mathcal{H} E \Phi+1)^{4}} \\
& -\frac{\mathcal{H}^{3} \Phi^{7} E^{3} R e}{\left(\Phi^{2}-2\right)^{2}(\mathcal{H} E \Phi+1)^{4}}-3 \frac{\mathcal{H}^{2} \Phi^{6} E^{2} R e}{\left(\Phi^{2}-2\right)^{2}(\mathcal{H} E \Phi+1)^{4}} \\
& -3 \frac{\mathcal{H} \Phi^{5} E R e}{\left(\Phi^{2}-2\right)^{2}(\mathcal{H} E \Phi+1)^{4}}+4 \frac{\mathcal{H}^{3} \Phi^{5} E^{3} R e}{\left(\Phi^{2}-2\right)^{2}(\mathcal{H} E \Phi+1)^{4}} \\
& +12 \frac{\mathcal{H}^{2} \Phi^{4} R e E^{2}}{\left(\Phi^{2}-2\right)^{2}(\mathcal{H} E \Phi+1)^{4}}+12 \frac{\mathcal{H} \Phi^{3} E R e}{\left(\Phi^{2}-2\right)^{2}(\mathcal{H} E \Phi+1)^{4}}
\end{aligned}
$$




$$
\begin{aligned}
& -4 \frac{\mathcal{H}^{3} \Phi^{3} R e E^{3}}{\left(\Phi^{2}-2\right)^{2}(\mathcal{H} E \Phi+1)^{4}}-12 \frac{\mathcal{H}^{2} \Phi^{2} R e E^{2}}{\left(\Phi^{2}-2\right)^{2}(\mathcal{H} E \Phi+1)^{4}} \\
& -12 \frac{\mathcal{H} E \Phi R e}{\left(\Phi^{2}-2\right)^{2}(\mathcal{H} E \Phi+1)^{4}}-\frac{\Phi^{4} R e}{\left(\Phi^{2}-2\right)^{2}(\mathcal{H} E \Phi+1)^{4}} \\
& -4 \frac{R e}{\left(\Phi^{2}-2\right)^{2}(\mathcal{H} E \Phi+1)^{4}}+4 \frac{\Phi^{2} R e}{\left(\Phi^{2}-2\right)^{2}(\mathcal{H} E \Phi+1)^{4}} \\
& -1 / 6 \frac{\mathcal{H}^{7} \Phi^{4} \operatorname{Re} \zeta^{4} E^{4} \mathcal{H}_{X X X X}}{C a\left(\Phi^{2}-2\right)^{2}(\mathcal{H} E \Phi+1)^{4}}-2 / 3 \frac{\mathcal{H}^{6} \Phi^{3} \operatorname{Re} \zeta^{4} E^{3} \mathcal{H}_{X X X X}}{C a\left(\Phi^{2}-2\right)^{2}(\mathcal{H} E \Phi+1)^{4}} \\
& +8 / 3 \frac{\mathcal{H}^{6} \Phi \operatorname{Re} \zeta^{4} E^{3} \mathcal{H}_{X X X X}}{C a\left(\Phi^{2}-2\right)^{2}(\mathcal{H} E \Phi+1)^{4}}-2 / 3 \frac{\mathcal{H}^{4} \Phi R e \zeta^{4} E \mathcal{H}_{X X X X}}{C a\left(\Phi^{2}-2\right)^{2}(\mathcal{H} E \Phi+1)^{4}} \\
& -2 \frac{\mathcal{H}^{2} \Phi^{3} \zeta \Gamma E^{4} \mathcal{H}_{X}{ }^{2}}{\left(\Phi^{2}-2\right)^{2}(\mathcal{H} E \Phi+1)^{4}}-12 \frac{\mathcal{H} \Phi^{3} E^{2} \zeta \Gamma \mathcal{H}_{X X}}{\left(\Phi^{2}-2\right)^{2}(\mathcal{H} E \Phi+1)^{4}} \\
& +12 \frac{\Phi^{3} \zeta \Gamma E^{2} \mathcal{H}_{X}{ }^{2}}{\left(\Phi^{2}-2\right)^{2}(\mathcal{H} E \Phi+1)^{4}}+2 \frac{\mathcal{H}^{3} \Phi^{3} \zeta \Gamma E^{4} \mathcal{H}_{X X}}{\left(\Phi^{2}-2\right)^{2}(\mathcal{H} E \Phi+1)^{4}} \\
& +2 / 3 \frac{\mathcal{H}^{7} \Phi^{2} \operatorname{Re} \zeta^{4} E^{4} \mathcal{H}_{X X X X}}{C a\left(\Phi^{2}-2\right)^{2}(\mathcal{H} E \Phi+1)^{4}}-\frac{\mathcal{H}^{5} \Phi^{2} \operatorname{Re} \zeta^{4} E^{2} \mathcal{H}_{X X X X}}{C a\left(\Phi^{2}-2\right)^{2}(\mathcal{H} E \Phi+1)^{4}} \\
& -8 \frac{\zeta \Gamma E \mathcal{H}_{X}{ }^{2}}{\left(\Phi^{2}-2\right)^{2}(\mathcal{H} E \Phi+1)^{4} \mathcal{H}}-4 \frac{\mathcal{H}^{5} \zeta^{4} R e E^{2} \mathcal{H}_{X X X X}}{C a\left(\Phi^{2}-2\right)^{2}(\mathcal{H} E \Phi+1)^{4} \Phi^{2}} \\
& -2 \frac{\mathcal{H}^{3} \zeta^{4} \operatorname{Re} \mathcal{H}_{X X} \Phi_{X}{ }^{2}}{C a\left(\Phi^{2}-2\right)^{2}(\mathcal{H} E \Phi+1)^{4} \Phi^{2}}+2 \frac{\mathcal{H}^{2} \operatorname{Re} \zeta^{4} \mathcal{H}_{X X X} \mathcal{H}_{X}}{C a\left(\Phi^{2}-2\right)^{2}(\mathcal{H} E \Phi+1)^{4} \Phi^{2}} \\
& +1 / 2 \frac{\mathcal{H}^{3} \Phi^{7} E^{4} \zeta \Gamma \mathcal{H}_{X X}}{\left(\Phi^{2}-2\right)^{2}(\mathcal{H} E \Phi+1)^{4}}-1 / 2 \frac{\mathcal{H}^{2} \Phi^{7} E^{4} \zeta \Gamma \mathcal{H}_{X}{ }^{2}}{\left(\Phi^{2}-2\right)^{2}(\mathcal{H} E \Phi+1)^{4}} \\
& +8 \frac{\mathcal{H}^{3} \zeta^{4} \operatorname{Re}_{\mathcal{H}_{X X} \Phi_{X}{ }^{2}}}{C a\left(\Phi^{2}-2\right)^{2}(\mathcal{H} E \Phi+1)^{4} \Phi^{4}}-2 \frac{\mathcal{H}^{2} \operatorname{Re}^{4} \mathcal{H}_{X X X} \mathcal{H}_{X}}{C a\left(\Phi^{2}-2\right)^{2}(\mathcal{H} E \Phi+1)^{4} \Phi^{4}} \\
& +4 \frac{\mathcal{H}^{5} \zeta^{4} \operatorname{Re} E^{2} \mathcal{H}_{X X X X}}{C a\left(\Phi^{2}-2\right)^{2}(\mathcal{H} E \Phi+1)^{4}}-1 / 2 \frac{\mathcal{H}^{2} \operatorname{Re} \zeta^{4} \mathcal{H}_{X X X} \mathcal{H}_{X}}{C a\left(\Phi^{2}-2\right)^{2}(\mathcal{H} E \Phi+1)^{4}} \\
& -2 / 3 \frac{\mathcal{H}^{7} \operatorname{Re} \zeta^{4} E^{4} \mathcal{H}_{X X X X}}{C a\left(\Phi^{2}-2\right)^{2}(\mathcal{H} E \Phi+1)^{4}}-8 \frac{\mathcal{H}^{3} \zeta^{4} \operatorname{Re} \mathcal{H}_{X X} \Phi_{X}{ }^{2}}{C a\left(\Phi^{2}-2\right)^{2}(\mathcal{H} E \Phi+1)^{4} \Phi^{6}} \\
& +2 \frac{\mathcal{H}^{2} \Phi^{6} E^{3} \zeta \Gamma \mathcal{H}_{X X}}{\left(\Phi^{2}-2\right)^{2}(\mathcal{H} E \Phi+1)^{4}}-2 \frac{\mathcal{H} \Phi^{6} E^{3} \zeta \Gamma \mathcal{H}_{X}{ }^{2}}{\left(\Phi^{2}-2\right)^{2}(\mathcal{H} E \Phi+1)^{4}}
\end{aligned}
$$




$$
\begin{aligned}
& +2 \frac{\mathcal{H}^{2} \Phi^{5} E^{4} \zeta \Gamma \mathcal{H}_{X}{ }^{2}}{\left(\Phi^{2}-2\right)^{2}(\mathcal{H} E \Phi+1)^{4}}+3 \frac{\mathcal{H} \Phi^{5} E^{2} \zeta \Gamma \mathcal{H}_{X X}}{\left(\Phi^{2}-2\right)^{2}(\mathcal{H} E \Phi+1)^{4}} \\
& -2 \frac{\mathcal{H}^{3} \Phi^{5} E^{4} \zeta \Gamma \mathcal{H}_{X X}}{\left(\Phi^{2}-2\right)^{2}(\mathcal{H} E \Phi+1)^{4}}-3 \frac{\Phi^{5} E^{2} \zeta \Gamma \mathcal{H}_{X}{ }^{2}}{\left(\Phi^{2}-2\right)^{2}(\mathcal{H} E \Phi+1)^{4}} \\
& -8 \frac{\mathcal{H}^{2} \Phi^{4} \zeta \Gamma E^{3} \mathcal{H}_{X X}}{\left(\Phi^{2}-2\right)^{2}(\mathcal{H} E \Phi+1)^{4}}+8 \frac{\mathcal{H} \Phi^{4} E^{3} \zeta \Gamma \mathcal{H}_{X}{ }^{2}}{\left(\Phi^{2}-2\right)^{2}(\mathcal{H} E \Phi+1)^{4}} \\
& -2 \frac{\Phi^{4} \zeta \Gamma E \mathcal{H}_{X}{ }^{2}}{\left(\Phi^{2}-2\right)^{2}(\mathcal{H} E \Phi+1)^{4} \mathcal{H}}+2 \frac{\Phi^{4} \zeta \Gamma E \mathcal{H}_{X X}}{\left(\Phi^{2}-2\right)^{2}(\mathcal{H} E \Phi+1)^{4}} \\
& -2 \frac{\mathcal{H}^{3} \zeta^{4} \operatorname{Re} \mathcal{H}_{X X} \Phi_{X X}}{C a\left(\Phi^{2}-2\right)^{2}(\mathcal{H} E \Phi+1)^{4} \Phi^{3}}-4 \frac{\mathcal{H}^{3} \zeta^{4} \operatorname{Re} \mathcal{H}_{X X X} \Phi_{X}}{C a\left(\Phi^{2}-2\right)^{2}(\mathcal{H} E \Phi+1)^{4} \Phi^{3}} \\
& -8 / 3 \frac{\mathcal{H}^{4} \operatorname{Re} \zeta^{4} E \mathcal{H}_{X X X X}}{C a\left(\Phi^{2}-2\right)^{2}(\mathcal{H} E \Phi+1)^{4} \Phi^{3}}+12 \frac{\mathcal{H} \Phi E^{2} \zeta \Gamma \mathcal{H}_{X X}}{\left(\Phi^{2}-2\right)^{2}(\mathcal{H} E \Phi+1)^{4}} \\
& -12 \frac{\Phi \zeta \Gamma E^{2} \mathcal{H}_{X}{ }^{2}}{\left(\Phi^{2}-2\right)^{2}(\mathcal{H} E \Phi+1)^{4}}+8 / 3 \frac{\mathcal{H}^{4} \operatorname{Re} \zeta^{4} E \mathcal{H}_{X X X X}}{C a\left(\Phi^{2}-2\right)^{2}(\mathcal{H} E \Phi+1)^{4} \Phi} \\
& -8 / 3 \frac{\mathcal{H}^{6} \operatorname{Re} \zeta^{4} E^{3} \mathcal{H}_{X X X X}}{C a\left(\Phi^{2}-2\right)^{2}(\mathcal{H} E \Phi+1)^{4} \Phi}+\frac{\mathcal{H}^{3} \zeta^{4} \operatorname{Re} \mathcal{H}_{X X X} \Phi_{X}}{C a\left(\Phi^{2}-2\right)^{2}(\mathcal{H} E \Phi+1)^{4} \Phi} \\
& +1 / 2 \frac{\mathcal{H}^{3} \zeta^{4} \operatorname{Re} \mathcal{H}_{X X} \Phi_{X X}}{C a\left(\Phi^{2}-2\right)^{2}(\mathcal{H} E \Phi+1)^{4} \Phi}+8 \frac{\zeta \Gamma E \mathcal{H}_{X X}}{\left(\Phi^{2}-2\right)^{2}(\mathcal{H} E \Phi+1)^{4}} \\
& +8 \frac{\Phi^{2} \zeta \Gamma E \mathcal{H}_{X}{ }^{2}}{\left(\Phi^{2}-2\right)^{2}(\mathcal{H} E \Phi+1)^{4} \mathcal{H}}-8 \frac{\Phi^{2} \zeta \Gamma E \mathcal{H}_{X X}}{\left(\Phi^{2}-2\right)^{2}(\mathcal{H} E \Phi+1)^{4}} \\
& +8 \frac{\mathcal{H}^{2} \Phi^{2} \zeta \Gamma E^{3} \mathcal{H}_{X X}}{\left(\Phi^{2}-2\right)^{2}(\mathcal{H} E \Phi+1)^{4}}
\end{aligned}
$$




\section{Appendix $\mathrm{C}$}

\section{Simplified evolution equation}

$$
\begin{aligned}
& \mathcal{H}_{\tau}=1 / 2 \frac{\Gamma \mathcal{H}_{X X} \zeta}{\mathcal{H}(\mathcal{H} E+1)^{4}}-1 / 2 \frac{\Gamma \mathcal{H}_{X}^{2} \zeta}{\mathcal{H}^{2}(\mathcal{H} E+1)^{4}}-3 \frac{\Gamma \mathcal{H}_{X}{ }^{2} \zeta E^{2}}{(\mathcal{H} E+1)^{4}} \\
& -\frac{\mathcal{H}^{3} R e E^{3}}{(\mathcal{H} E+1)^{4}}-3 \frac{\mathcal{H}^{2} R e E^{2}}{(\mathcal{H} E+1)^{4}}-3 \frac{\mathcal{H} R e E}{(\mathcal{H} E+1)^{4}}-\frac{R e}{(\mathcal{H} E+1)^{4}} \\
& +1 / 2 \frac{\mathcal{H}^{3} \Gamma \mathcal{H}_{X X} \zeta E^{4}}{(\mathcal{H} E+1)^{4}}-1 / 2 \frac{\mathcal{H}^{2} \Gamma \mathcal{H}_{X}{ }^{2} \zeta E^{4}}{(\mathcal{H} E+1)^{4}}+2 \frac{\mathcal{H}^{2} \Gamma \mathcal{H}_{X X} \zeta E^{3}}{(\mathcal{H} E+1)^{4}} \\
& -2 \frac{\mathcal{H} \Gamma \mathcal{H}_{X}{ }^{2} \zeta E^{3}}{(\mathcal{H} E+1)^{4}}+3 \frac{\mathcal{H} \Gamma \mathcal{H}_{X X} \zeta E^{2}}{(\mathcal{H} E+1)^{4}}-2 \frac{\Gamma \mathcal{H}_{X}{ }^{2} \zeta E}{\mathcal{H}(\mathcal{H} E+1)^{4}} \\
& -\frac{\mathcal{H}^{2} \mathcal{H}_{X}{ }^{2} R e_{m} \zeta^{2} R e E}{(\mathcal{H} E+1)^{4}}-1 / 3 \frac{\mathcal{H}^{4} \mathcal{H}_{X X} R e_{m} \zeta^{2} R e E^{2}}{(\mathcal{H} E+1)^{4}} \\
& -1 / 3 \frac{\mathcal{H}^{3} \mathcal{H}_{X X} \operatorname{Re}_{m} \zeta^{2} \operatorname{Re} E}{(\mathcal{H} E+1)^{4}}+1 / 2 \frac{\mathcal{H}^{2} \mathcal{H}_{X X} M a \zeta R e E}{\operatorname{Pe}(\mathcal{H} E+1)^{4}} \\
& +1 / 6 \frac{\mathcal{H}^{4} \mathcal{H}_{X}{ }^{2} M a \zeta R e E^{4}}{\operatorname{Pe}(\mathcal{H} E+1)^{4}}+7 / 6 \frac{\mathcal{H}^{3} \mathcal{H}_{X X} M a \zeta R e E^{2}}{\operatorname{Pe}(\mathcal{H} E+1)^{4}} \\
& +2 / 3 \frac{\mathcal{H}^{3} \mathcal{H}_{X}{ }^{2} M a \zeta R e E^{3}}{\operatorname{Pe}(\mathcal{H} E+1)^{4}}+\frac{\mathcal{H H}_{X}{ }^{2} M a \zeta R e E}{\operatorname{Pe}(\mathcal{H} E+1)^{4}} \\
& +5 / 6 \frac{\mathcal{H}^{4} \mathcal{H}_{X X} M a \zeta R e E^{3}}{\operatorname{Pe}(\mathcal{H} E+1)^{4}}+3 / 2 \frac{\mathcal{H}^{2} \mathcal{H}_{X}^{2} M a \zeta R e E^{2}}{\operatorname{Pe}(\mathcal{H} E+1)^{4}} \\
& +1 / 6 \frac{\mathcal{H}^{5} \mathcal{H}_{X X} M a \zeta R e E^{4}}{\operatorname{Pe}(\mathcal{H} E+1)^{4}}+2 \frac{\Gamma \mathcal{H}_{X X} \zeta E}{(\mathcal{H} E+1)^{4}}
\end{aligned}
$$




\section{Appendix D}

\section{Perturbation growth rate}

$$
\frac{h_{p_{\tau}}}{h_{p}}=\mathcal{A} k^{4}+\mathcal{B} k^{2}+\mathcal{C}
$$

where,

$$
\begin{aligned}
\mathcal{A}= & -5 / 3 \frac{\zeta^{4} R e H^{6} E^{3}}{C a(H E+1)^{5}}-5 / 3 \frac{\zeta^{4} R e H^{5} E^{2}}{C a(H E+1)^{5}}-5 / 6 \frac{\zeta^{4} R e H^{4} E}{C a(H E+1)^{5}} \\
- & 1 / 6 \frac{\zeta^{4} R e H^{8} E^{5}}{C a(H E+1)^{5}}-5 / 6 \frac{\zeta^{4} R e H^{7} E^{4}}{C a(H E+1)^{5}}-1 / 6 \frac{\zeta^{4} R e H^{3}}{C a(H E+1)^{5}} \\
\mathcal{B}= & 1 / 3 \frac{H^{5} \zeta^{2} \operatorname{ReRe} E^{3}}{(H E+1)^{5}}+2 / 3 \frac{H^{4} \zeta^{2} R e R e_{m} E^{2}}{(H E+1)^{5}}+1 / 3 \frac{H^{3} \zeta^{2} R e R e_{m} E}{(H E+1)^{5}} \\
& -1 / 2 \frac{H^{2} \zeta R e M a E}{P e(H E+1)^{5}}-1 / 6 \frac{H^{6} \zeta M a R e E^{5}}{P e(H E+1)^{5}}-\frac{H^{5} \zeta M a R e E^{4}}{P e(H E+1)^{5}} \\
& -2 \frac{H^{4} \zeta M a R e E^{3}}{P e(H E+1)^{5}}-5 / 3 \frac{H^{3} \zeta R e M a E^{2}}{P e(H E+1)^{5}}-1 / 2 \frac{\zeta \Gamma}{H(H E+1)^{5}} \\
& -5 / 2 \frac{\zeta \Gamma E}{(H E+1)^{5}}-1 / 2 \frac{H^{4} \zeta \Gamma E^{5}}{(H E+1)^{5}}-5 / 2 \frac{H^{3} \zeta \Gamma E^{4}}{(H E+1)^{5}} \\
& -5 \frac{H^{2} \zeta \Gamma E^{3}}{(H E+1)^{5}}-5 \frac{H \zeta \Gamma E^{2}}{(H E+1)^{5}}
\end{aligned}
$$




$$
\begin{aligned}
& \mathcal{C}=\frac{\zeta H_{X}{ }^{2} \operatorname{Re} M a E}{\operatorname{Pe}(H E+1)^{5}}-\frac{\zeta^{4} R e H_{X X X} H_{X} H}{C a(H E+1)^{5}}+2 \frac{\zeta^{2} H_{X}{ }^{2} R e R e_{m} H^{2} E^{2}}{(H E+1)^{5}} \\
& -2 \frac{\zeta^{2} H_{X}{ }^{2} R e R e_{m} H E}{(H E+1)^{5}}-\frac{R e \zeta^{2} R e_{m} H_{X X} H^{3} E^{2}}{(H E+1)^{5}}-\frac{R e \zeta^{2} R e_{m} H_{X X} H^{2} E}{(H E+1)^{5}} \\
& -5 / 2 \frac{\zeta^{4} R e H_{X X X X} H^{6} E^{4}}{C a(H E+1)^{5}}-5 \frac{\zeta^{4} R e H_{X X X X} H^{5} E^{3}}{C a(H E+1)^{5}}-5 / 2 \frac{\zeta^{4} R e H_{X X X X} H^{3} E}{C a(H E+1)^{5}} \\
& -1 / 2 \frac{\zeta^{4} \operatorname{Re} H_{X X X X} H^{7} E^{5}}{C a(H E+1)^{5}}-5 \frac{\zeta^{4} \operatorname{Re} H_{X X X X} H^{4} E^{2}}{C a(H E+1)^{5}}+\frac{R e H^{3} E^{4}}{(H E+1)^{5}} \\
& +3 \frac{R e H E^{2}}{(H E+1)^{5}}+3 \frac{R e H^{2} E^{3}}{(H E+1)^{5}}+\frac{R e E}{(H E+1)^{5}}-1 / 2 \frac{\Gamma \zeta H_{X X}}{H^{2}(H E+1)^{5}} \\
& -5 \frac{\zeta^{4} \operatorname{Re} H_{X X X} H_{X} H^{2} E}{C a(H E+1)^{5}}-\frac{\zeta^{4} R e H_{X X X} H_{X} H^{6} E^{5}}{C a(H E+1)^{5}}-\frac{\zeta H_{X}^{2} R e M a H^{2} E^{3}}{P e(H E+1)^{5}} \\
& +\frac{13}{6} \frac{\operatorname{Re} \zeta M a H_{X X} H^{3} E^{3}}{\operatorname{Pe}(H E+1)^{5}}+5 / 6 \frac{\operatorname{Re} \zeta M a H_{X X} H^{4} E^{4}}{\operatorname{Pe}(H E+1)^{5}} \\
& +1 / 6 \frac{\operatorname{Re} \zeta M a H_{X X} H^{5} E^{5}}{\operatorname{Pe}(H E+1)^{5}}+5 / 2 \frac{\operatorname{Re} \zeta M a H_{X X} H^{2} E^{2}}{\operatorname{Pe}(H E+1)^{5}}+\frac{\operatorname{Re} \zeta M a H_{X X} H E}{\operatorname{Pe}(H E+1)^{5}} \\
& -10 \frac{\zeta^{4} R e H_{X X X} H_{X} H^{3} E^{2}}{C a(H E+1)^{5}}-5 \frac{\zeta^{4} R e H_{X X X} H_{X} H^{5} E^{4}}{C a(H E+1)^{5}}-10 \frac{\zeta^{4} R e H_{X X X} H_{X} H^{4} E^{3}}{C a(H E+1)^{5}} \\
& +10 \frac{\zeta H_{X}^{2} \Gamma E^{3}}{(H E+1)^{5}}-5 \frac{\Gamma \zeta H_{X X} E^{2}}{(H E+1)^{5}}+\frac{\zeta H_{X}^{2} \Gamma H^{2} E^{5}}{(H E+1)^{5}} \\
& +5 \frac{\zeta H_{X}^{2} \Gamma H E^{4}}{(H E+1)^{5}}+\frac{\zeta H_{X}^{2} \Gamma}{H^{3}(H E+1)^{5}}+5 \frac{\zeta H_{X}^{2} \Gamma E}{H^{2}(H E+1)^{5}} \\
& +10 \frac{\zeta H_{X}{ }^{2} \Gamma E^{2}}{H(H E+1)^{5}}-5 / 2 \frac{\Gamma \zeta H_{X X} E}{H(H E+1)^{5}}-5 \frac{\Gamma \zeta H_{X X} H E^{3}}{(H E+1)^{5}} \\
& -1 / 2 \frac{\Gamma \zeta H_{X X} H^{3} E^{5}}{(H E+1)^{5}}-5 / 2 \frac{\Gamma \zeta H_{X X} H^{2} E^{4}}{(H E+1)^{5}}-1 / 2 \frac{\zeta^{4} R e H_{X X X X} H^{2}}{C a(H E+1)^{5}}
\end{aligned}
$$

